TRANSACTIONS OF THE

AMERICAN MATHEMATICAL SOCIETY

Volume 356, Number 12, Pages 4969-5023

S 0002-9947(04)03477-4

Article electronically published on April 27, 2004

\title{
CROSS CHARACTERISTIC REPRESENTATIONS OF EVEN CHARACTERISTIC SYMPLECTIC GROUPS
}

\author{
ROBERT M. GURALNICK AND PHAM HUU TIEP
}

\begin{abstract}
We classify the small irreducible representations of $S p_{2 n}(q)$ with $q$ even in odd characteristic. This improves even the known results for complex representations. The smallest representation for this group is much larger than in the case when $q$ is odd. This makes the problem much more difficult.
\end{abstract}

\section{INTRODUCTION}

In [LaS], Landazuri and Seitz gave lower bounds for irreducible representations of Chevalley groups in cross characteristic, and these lower bounds were improved further by Seitz and Zalesskii in [SZ]. The Landazuri-Seitz-Zalesskii bounds have proved to be useful in many applications. In further applications, particularly in various problems related to the classification of maximal subgroups of finite classical groups (cf. for instance GPPS, [MT1, [MMT]), in the minimal polynomial problem (see e.g. GMST), in the determination of submodule structure of small rank permutation modules ([LST], [ST]), and in computer programs to recognize linear groups of moderate degree, it is also important to identify the modules which have dimension close to the smallest possible dimension and to prove that there are no irreducible modules with dimension in a certain range above it. This was done in GPPS and [GT] for $S L_{n}(q)$. Further improvements were obtained by Brundan and Kleshchev $[\mathrm{BrK}$. Hiss and Malle $[\mathrm{HM}]$ have obtained results similar to $[\mathrm{GT}$ for unitary groups, and their results have recently been improved in [GMST]. The case of $S p_{2 n}(q)$ with odd $q$ has recently been done in [GMST].

In this paper, we consider representations of the finite symplectic groups $G=$ $S p_{2 n}(q)$ with $n \geq 2$ and $q$ even over an algebraically closed field $\mathbb{F}$ of characteristic $\ell \neq 2$. Landazuri and Seitz $[\mathrm{LaS}]$ had already shown that the smallest dimension

Received by the editors June 5, 2002 and, in revised form, July 29, 2003.

2000 Mathematics Subject Classification. Primary 20C33, 20G05, 20C20, 20 G40.

Key words and phrases. Finite symplectic group, Weil representation, cross characteristic representation, low dimensional representation.

Part of this paper was written while the authors were participating in the Symposium "Groups, Geometries, and Combinatorics", London Mathematical Society, July 16-26, 2001, Durham, England. It is a pleasure to thank the organizers A. A. Ivanov, M. W. Liebeck, and J. Saxl for their generous hospitality and support. The authors are also thankful to the referee for helpful comments on the paper.

The authors gratefully acknowledge the support of the NSF (grants DMS-9970305, DMS0140578 and DMS-0070647) and the NSA. 
of nontrivial cross characteristic representations of $G$ is

$$
d(G):=\left(q^{n}-1\right)\left(q^{n}-q\right) / 2(q+1) .
$$

Decomposition matrices are known in the case of $S p_{4}(q)$ [Wh1, and in the case of unipotent blocks of $S p_{6}(q)$ Wh2].

One of the principal differences between the cases of $S L_{n}(q), S U_{n}(q)$, and $S p_{2 n}(q)$ with odd $q$ that have been treated before and the case we are considering here is that the minimum dimension of cross characteristic representations of $G=S p_{2 n}(q)$ with $q$ even is not small (compare $d(G)$ to $\left(q^{n}-1\right) / 2$ which is the bound for $S p_{2 n}(q)$ when $q$ is odd). Moreover, in all the previously-studied cases one has obvious candidates for small representations which are the well-known Weil modules (see e.g. GMST). In our case, even the complex irreducible representations of small degree of $G$ are much less understood, and their construction is far from being obvious. (Notice that the classification of small complex representations as given in [TZ1] only went up to $d(G)\left(1+O\left(q^{-n}\right)\right)$.) It turns out, however, that in many respects they still resemble the Weil representations that occur in the previous cases. In fact, these representations of $G$ come from Weil representations of $S L_{2 n}(q)$, in which case we call them (complex) linear-Weil representations of $G$, or from Weil representations of $S U_{2 n}(q)$, in which case we call them (complex) unitary-Weil representations of $G$. A good construction and a better understanding of these complex representations are furnished using the concept of Howe's dual pairs Hw2. This concept is fairly well-known for finite groups of Lie type in odd characteristic; however in the case of Lie type groups in characteristic 2 this has been worked out first in [T]. When reduced modulo $\ell$, these complex Weil representations produce $\left((q-1)_{\ell^{\prime}}+3\right) / 2$ irreducible linear-Weil representations and $\left((q+1)_{\ell^{\prime}}+3\right) / 2$ irreducible unitary-Weil representations in characteristic $\ell$. Formal definitions and properties of complex and modular Weil representations of $G$ are described in $\S \S 3,7$. For the reader's convenience, we collect in Table I the relevant information about complex and modular Weil characters of $S p_{2 n}(q)$ (extracted from Corollaries 7.5 and 7.10). In this table, $\widehat{\chi}$ denotes the restriction of a character $\chi$ to $\ell^{\prime}$-elements, and $N_{\ell^{\prime}}$ denotes the $\ell^{\prime}$-part of an integer $N$.

As in the case of odd $q$, Weil representations of $G$ can also be characterized by certain local properties, $\left(\mathcal{W}_{1}\right)$ and $\left(\mathcal{W}_{2}^{\varepsilon}\right)$, which are studied in $\S \S 3,5$. The most transparent one, $\left(\mathcal{W}_{2}^{\varepsilon}\right)$, means that the representation in question does not afford a $P_{2}$-orbit of $Z_{2}$-characters of length $q(q-1)(q-\varepsilon) / 2$ (which correspond to all quadratic forms of rank 2 and type $-\varepsilon$ on $\mathbb{F}_{q}^{2}$ ). Here $P_{2}$ is the stabilizer in $G$ of a 2-dimensional totally isotropic subspace of the natural module $\mathbb{F}_{q}^{2 n}$ of $G$, and $Z_{2}=Z\left(O_{2}\left(P_{2}\right)\right)$.

We will exploit the method that has been first developed in GMST, which is to analyze modules with various local properties, and by restricting to various families of subgroups which contain a conjugate of every element of the group, we can determine the Brauer character of the module in question. However, another principal difference between our case and the case of $S p_{2 n}(q)$ with odd $q$ considered in [GMST] is that $G$ contains some semisimple elements (namely elements of tori of order $q^{n} \pm 1$ ) which cannot be covered by subgroups good enough for the purposes of this "gluing" method. To cover those elements we rely heavily upon DeligneLusztig theory $[\mathrm{L}$, and upon some fundamental results of Broué and Michel [BM] on unions of $\ell$-blocks and of Geck and Hiss [GH] on basic sets of Brauer characters, which also depend on Deligne-Lusztig theory. 
TABLE I. Weil characters of $S p_{2 n}(q)$ ( $q$ even, $\left.n \geq 2, \ell \neq 2\right)$

\begin{tabular}{|c||c|c|}
\hline $\begin{array}{c}\text { Complex linear-Weil } \\
\text { characters }\end{array}$ & Degree & $\begin{array}{c}\ell \text {-modular linear-Weil } \\
\text { characters }\end{array}$ \\
\hline \hline$\rho_{n}^{1}$ & $\frac{\left(q^{n}+1\right)\left(q^{n}-q\right)}{2(q-1)}$ & $\hat{\rho}_{n}^{1}-\left\{\begin{array}{cc|}1, \quad \ell \mid q^{n}-1 \\
0,-1 \\
0, & \text { otherwise }\end{array}\right.$ \\
\hline$\rho_{n}^{2}$ & $\frac{\left(q^{n}-1\right)\left(q^{n}+q\right)}{2(q-1)}$ & $\hat{\rho}_{n}^{2}-\left\{\begin{array}{cc|}1, \quad \ell \mid\left(q^{n}+1\right), \\
0, & \text { otherwise }\end{array}\right.$ \\
\hline $\begin{array}{c}\tau_{n}^{i}, \\
1 \leq i \leq(q-2) / 2\end{array}$ & $\frac{q^{2 n}-1}{q-1}$ & $1 \leq i \leq\left((q-1)_{\ell^{\prime}}-1\right) / 2$ \\
\hline $\begin{array}{c}\text { Complex unitary-Weil } \\
\text { characters }\end{array}$ & Degree & $\begin{array}{c}\ell \text {-modular unitary-Weil } \\
\text { characters }\end{array}$ \\
\hline \hline$\alpha_{n}$ & $\frac{\left(q^{n}-1\right)\left(q^{n}-q\right)}{2(q+1)}$ & $\widehat{\alpha}_{n}$ \\
\hline$\beta_{n}$ & $\frac{\left(q^{n}+1\right)\left(q^{n}+q\right)}{2(q+1)}$ & $\widehat{\beta}_{n}-\left\{\begin{array}{cc}1, \quad \ell \mid(q+1), \\
0, & \text { otherwise }\end{array}\right.$ \\
\hline $\begin{array}{c}\zeta_{n}^{i}, \\
1 \leq i \leq q / 2\end{array}$ & $\frac{q^{2 n}-1}{q+1}$ & $1 \leq i \leq\left((q+1)_{\ell^{\prime}}-1\right) / 2$ \\
\hline
\end{tabular}

The main results of the paper are the following two theorems. In Theorem 1.1 we define $\alpha=19 / 15$ if $(n, q)=(5,2), \alpha=2$ if $(n, q)=(5,4)$ or $(6,2)$, and $\alpha=0$ otherwise.

Theorem 1.1. Let $G=S p_{2 n}(q)$ with $q$ even and $n \geq 2$. Let $V$ be an absolutely irreducible $G$-module in characteristic $\ell \neq 2$ of dimension less than

$$
\mathfrak{d}(n, q):=\left\{\begin{array}{cc}
q^{2}(q-1), & n=2, \\
21, & (n, q)=(3,2), \\
q^{2}\left(q^{3}-1\right), & n=3, q>2, \\
203, & (n, q)=(4,2), \\
\left(q^{4}-1\right)\left(q^{3}-1\right) q^{2}, & n=4, q>2, \\
\left(\frac{\left(q^{n-1}+1\right)\left(q^{n-2}-q\right)}{q^{2}-1}-1-\alpha\right) \frac{q^{n-1}\left(q^{n-1}-1\right)(q-1)}{2}, & n \geq 5 .
\end{array}\right.
$$

Then $V$ is either the trivial module or a Weil module.

Theorem 1.2. Let $G=S p_{2 n}(q), q$ be even, $n \geq 2,(n, q) \neq(2,2),(3,2)$. Let $V$ be an absolutely irreducible $G$-module in characteristic $\ell \neq 2$ that satisfies $\left(\mathcal{W}_{2}^{\varepsilon}\right)$ for some $\varepsilon= \pm$. Then one of the following holds:

(i) $V$ is the trivial module.

(ii) $\varepsilon=+$ and $V$ is a linear-Weil module.

(iii) $\varepsilon=-$ and $V$ is a unitary-Weil module.

Observe that if $n \geq 5$, then $\mathfrak{d}(n, q)=\frac{1}{2} q^{4 n-6}\left(1-q^{-1}+O\left(q^{-2}\right)\right)$. In the meantime, Corollary 6.2 shows that $G$ has a complex irreducible non-Weil character of degree $D(n, q)=\frac{1}{2} q^{4 n-6}\left(1+q^{-4}+O\left(q^{-5}\right)\right)$. Hence the bound $\mathfrak{d}(n, q)$ given in Theorem 1.1 is the asymptotically correct bound when $n \geq 5$. We emphasize that Theorem 1.1 is proved relying upon Theorem 1.2 Moreover, Theorem 1.2 is also used in applications to identify the representations $V$ in question via the local property $\left(\mathcal{W}_{2}^{\varepsilon}\right)$, when a priori no upper bound on $\operatorname{dim}(V)$ is given. The results of the paper 
have already been used in MT2 to determine irreducible tensor products of cross characteristic representations of $S p_{2 n}(q)$ with $q$ even.

The orthogonal groups will be treated in a forthcoming paper.

Notation. Throughout the paper, $q$ is a power of $2, G=S p_{2 n}(q), \mathbb{F}$ is an algebraically closed field of characteristic $\ell \neq 2, I_{n}$ is the $n \times n$ identity matrix, and $J_{n}=\left(\begin{array}{cc}0 & I_{n} \\ I_{n} & 0\end{array}\right)$. Furthermore, $M=\mathbb{F}_{q}^{2 n}$ is equipped with a $G$-invariant nondegenerate symplectic form $(\cdot, \cdot)$, which has $J_{n}$ as Gram matrix in a fixed basis $\left(e_{1}, \ldots, e_{n}, f_{1}, \ldots, f_{n}\right)$. By a standard subgroup $S p_{2 j}(q)$ in $G$ we mean the pointwise stabilizer of a nondegenerate $(2 n-2 j)$-dimensional subspace of $M$. Also, $P_{j}$ is the stabilizer of a $j$-dimensional totally isotropic subspace in $M, Q_{j}=O_{p}\left(P_{j}\right)$, $Z_{j}=Z\left(Q_{j}\right)$ if $j>1$ and $Z_{1}=Z\left(P_{1}^{\prime}\right)$, and $L_{j} \simeq S p_{2 n-2 j}(q) \times G L_{j}(q)$ is the Levi subgroup. Next, $H_{d} \simeq S p_{2 d}(q) \times S p_{2 n-2 d}(q)$ is the stabilizer of a $2 d$-dimensional nondegenerate subspace of $M$. Let $M_{m, n}(q)$ be the set of $m \times n$-matrices over $\mathbb{F}_{q}$, $M_{n}(q)=M_{n, n}(q), \mathcal{H}_{n}(q)=\left\{X \in M_{n}(q) \mid{ }^{t} X+X=0\right\}, \mathcal{H}_{n}^{0}(q)=\left\{X \in \mathcal{H}_{n}(q) \mid\right.$ $X$ has zero diagonal $\}, \mathcal{F}_{n}(q)=M_{n}(q) / \mathcal{H}_{n}(q)$. If $\chi$ is a class function of $G$, then $\hat{\chi}$ is the restriction of $\chi$ to $\ell^{\prime}$-elements. The symbol $G L_{n}^{\alpha}(q)$ stands for $G L_{n}(q)$ if $\alpha=+$ and $G U_{n}(q)$ if $\alpha=-$. We fix a primitive $(q-1)^{\text {th }}$ root $\delta$ of unity in $\mathbb{F}_{q}$, and a primitive $(q-1)^{\text {th }}$ root $\tilde{\delta}$ of unity in $\mathbb{C}$. Furthermore, we fix a primitive $(q+1)^{\text {th }}$ root $\xi$ of unity in $\mathbb{F}_{q^{2}}$, and a primitive $(q+1)^{\text {th }}$ root $\tilde{\xi}$ of unity in $\mathbb{C}$. If $N$ is an integer and $r$ is a prime, then $N_{r^{\prime}}$ denotes the $r^{\prime}$-part of $N$. If $N$ is a prime power, then $[N]$ denotes the elementary abelian group of order $N$.

All Brauer characters are meant to be in characteristic $\ell$. If $A, B, C$ are $\mathbb{F} X$ modules for a group $X$, then we will use the notation $C=A+B$ to indicate that this is true in the Grothendieck group $G_{0}(X)$. Abusing notation, sometimes we will denote an $\mathbb{F} X$-module and its Brauer character by the same letter.

\section{Preliminaries}

We will frequently use the following version of [GMST Lemma 4.2]:

Lemma 2.1. Let $V$ be an $\mathbb{F} G$-module with $C_{V}(G)=0$ and let $U$ be an $L_{1}$ composition factor of $C_{V}\left(Q_{1}\right)$. Then $U$ is also an $L_{1}$-composition factor of $\left[V, Q_{1}\right]$.

Proof. We may assume that $P_{1}=\operatorname{Stab}_{G}\left(\left\langle e_{1}\right\rangle_{\mathbb{F}_{q}}\right)$. Let $g \in G$ be the element that flips $e_{1}$ and $f_{1}$ and fixes all $e_{i}$ and $f_{i}$ with $i \geq 2$. Then $L_{1}=S t a b_{G}\left(\left\langle e_{1}\right\rangle_{\mathbb{F}_{q}},\left\langle f_{1}\right\rangle_{\mathbb{F}_{q}}\right)$ normalizes both $Q_{1}$ and $Q_{1}^{g}$. Thus $N_{G}\left(\left\langle Q_{1}, Q_{1}^{g}\right\rangle\right)$ contains $\left\langle Q_{1}, Q_{1}^{g}\right\rangle L_{1}$ and therefore equals $G$; in other words $\left\langle Q_{1}, Q_{1}^{g}\right\rangle \triangleleft G$. But $G$ is simple, hence $\left\langle Q_{1}, Q_{1}^{g}\right\rangle=G$. Notice also that $g$ centralizes $L_{1}$. Therefore, our statement follows from GMST, Lemma $4.2]$.

The next result gives families of subgroups which contain a conjugate of every element in $G$. If $k l=n$, then $S p_{2 k}\left(q^{l}\right)$ naturally embeds in $G$ by viewing the natural $2 k$-dimensional module over $\mathbb{F}_{q^{l}}$ as a $2 n$-dimensional vector space over $\mathbb{F}_{q}$. For each $\varepsilon= \pm, T_{\varepsilon} \simeq \mathbb{Z}_{q^{n}-\varepsilon}$ is a torus of $S L_{2}\left(q^{n}\right)$ naturally embedded in $G$.

Lemma 2.2. Let $g \in G=S p_{2 n}(q)$ with $n \geq 3$. Then a $G$-conjugate of $g$ is contained in at least one of the following subgroups:

(i) $P_{j}$ with $1 \leq j \leq n-1, H_{d}$ with $1 \leq d \leq n / 2$, and $S p_{4}\left(q^{n / 2}\right)$, provided that $n$ is even; 
(ii) $P_{j}$ with $1 \leq j \leq n-1, H_{d}$ with $1 \leq d \leq n / 2$, and $S p_{2 k}\left(q^{n / k}\right)$, provided that $n$ is odd and divisible by $k>1$;

(iii) $P_{j}$ with $1 \leq j \leq n-1, H_{d}$ with $1 \leq d \leq n / 2, T_{+}$, and $T_{-}$, provided that $n$ is an odd prime.

Proof. 1) First consider the case when a conjugate of $g$ is contained in $P_{n}$. We claim that either a conjugate of $g$ is contained in some $P_{i}$ with $1 \leq i \leq n-1$, or $g$ is conjugate to an element of $T_{+}$, or $n$ is even and a conjugate of $g$ is contained in $S p_{4}\left(q^{n / 2}\right)$. Indeed, we may assume that $g$ stabilizes $U:=\left\langle e_{1}, \ldots, e_{n}\right\rangle_{\mathbb{F}_{q}}$. Let $g=s u$, where $s$ is the semisimple part and $u$ is the unipotent part of $g$. Since $u$ is a power of $g, u(U)=U$. If $\left.u\right|_{U} \neq 1_{U}$, then $g$ fixes $C_{U}(u)$, which is clearly a nonzero totally singular subspace and of dimension $j<n$, whence a conjugate of $g$ is contained in $P_{j}$. Assume that $u=1$ on $U$. If $\left.s\right|_{U}$ is not irreducible, then $s$ (and $g$ as well, since $u=1$ on $U$ ) fixes a proper subspace $U^{\prime}$ of $U$, and so a conjugate of $g$ is contained in $P_{j^{\prime}}$ with $j^{\prime}=\operatorname{dim}\left(U^{\prime}\right)<n$. Assume $\left.s\right|_{U}$ is irreducible. Then $\left.s\right|_{U}$ is conjugate (in the algebraic group $\left.S L_{n}\left(\overline{\mathbb{F}}_{q}\right)\right)$ to $\operatorname{diag}\left(\sigma, \sigma^{2}, \ldots, \sigma^{q^{n-1}}\right.$ ) with $\sigma^{q^{n}-1}=1$ but $\sigma^{q^{k}} \neq \sigma$ for $0<k<n$. If the $s$-module $U$ is not self-dual, then $C_{G}(s)$ is conjugate to $T_{+}$and so a conjugate of $g$ is contained in $T_{+}$. Assume the $s$-module $U$ is self-dual. In this case there must be some $k, 0 \leq k<n$, such that $\sigma^{q^{k}}=\sigma^{-1}$. It follows that $n$ is even, $C_{G}(s) \simeq G U_{2}\left(q^{n / 2}\right)<S p_{4}\left(q^{n / 2}\right)$, whence $g$ is conjugate to an element of $S p_{4}\left(q^{n / 2}\right)$.

2) By [GMST Lemma 4.4], a conjugate of any $g \in G$ is contained in some $P_{i}$, $H_{d}$, or $S L_{2}\left(q^{n}\right)$. Observe that if $x \in S L_{2}\left(q^{n}\right)$, then either $x \in P_{n}$, or $x \in T_{-}$. Hence a conjugate of any $g \in G$ is contained in some $P_{i}, H_{d}$, or $T_{-}$.

3) Assume $n$ is even. Then we may embed $T_{ \pm} \hookrightarrow S L_{2}\left(q^{n}\right) \hookrightarrow S p_{4}\left(q^{n / 2}\right)$. Hence the results of 1) and 2) yield statement (i).

Assume $n$ is odd and divisible by some $k>1$. Then we may embed $T_{ \pm} \hookrightarrow$ $S L_{2}\left(q^{n}\right) \hookrightarrow S p_{2 k}\left(q^{n / k}\right)$. Hence the results of 1) and 2) yield statement (ii).

Finally, 1) and 2) also imply statement (iii) in the case when $n$ is an odd prime.

Let $X$ be any finite group with a normal $\ell^{\prime}$-subgroup $Q$. If $V$ is any $\mathbb{F} X$-module and $\lambda \in \operatorname{IBr}_{\ell}(Q)$, then we will denote by $V_{\lambda}$ the $\lambda$-homogeneous component of $\left.V\right|_{Q}$, and let $I_{\lambda}:=\operatorname{Stab}_{X}(\lambda)$. Next, if $\varphi$ is a virtual Brauer character of $X$, then we may assume that $\varphi$ is afforded by $V-U$, where $V$ and $U$ are $\mathbb{F} X$-modules. In this case we may consider the virtual Brauer character of $I_{\lambda}$ afforded by $V_{\lambda}-U_{\lambda}$. This character does not depend on the choice of $V, U$ by the following lemma, hence we may talk about the virtual $I_{\lambda}$-module $(V-U)_{\lambda}:=V_{\lambda}-U_{\lambda}$ and call it the $\lambda$-homogeneous component of $\varphi$.

Lemma 2.3. Let $U, V, U^{\prime}, V^{\prime}$ be $\mathbb{F} X$-modules such that $V-U=V^{\prime}-U^{\prime}$. Then the virtual $I_{\lambda}$-characters afforded by $V_{\lambda}-U_{\lambda}$ and by $V_{\lambda}^{\prime}-U_{\lambda}^{\prime}$ are equal.

Proof. It suffices to show that the virtual Brauer characters in question take the same value at any $\ell^{\prime}$-element $g \in I_{\lambda}$. Replacing $X$ by $Y:=Q\langle g\rangle$, we may consider $U, V, U^{\prime}, V^{\prime}$ as complex $Y$-modules. By assumption, the complex $Y$-modules $V+U^{\prime}$ and $V^{\prime}+U$ have same character, whence they are equivalent. It follows that their $\lambda$-homogeneous components $\left(V+U^{\prime}\right)_{\lambda}=V_{\lambda}+U_{\lambda}^{\prime}$ and $\left(V^{\prime}+U\right)_{\lambda}=V_{\lambda}^{\prime}+U_{\lambda}$ are equivalent. Thus the traces of $g$ acting on $V_{\lambda}+U_{\lambda}^{\prime}$ and $V_{\lambda}^{\prime}+U_{\lambda}$ are equal. Consequently, the virtual traces of $g$ on $V_{\lambda}-U_{\lambda}$ and $V_{\lambda}^{\prime}-U_{\lambda}^{\prime}$ are equal, as stated. 
We will also need the following statement:

Lemma 2.4. Let $r$ be a prime and let $Q$ be a normal extraspecial $r$-subgroup of order $r^{1+2 n}$ of a finite group $X$. Suppose that $\chi$ is an irreducible complex character of $X$ of degree $r^{n}$ such that $\left.\chi\right|_{Q} \in \operatorname{Irr}(Q)$. Then for any $g \in X,|\chi(g)|^{2}=\left|C_{Q / Z(Q)}(g)\right|$ if $g$ acts trivially on the complete inverse image of $C_{Q / Z(Q)}(g)$ in $Q$, and $\chi(g)=0$ otherwise.

Proof. The statement is well known; cf. for instance [Is, Theorem (3.5)]. Even though it was assumed in Is that $r>2$, the argument given there goes through for any $r$. For completeness we give an outline of the argument. Denote $Z:=Z(Q)$, $\bar{C}:=C_{Q / Z}(g)$, and let $C$ be the complete inverse image of $\bar{C}$ in $Q$. It is clear that $\left.\chi\right|_{Z}=r^{n} \lambda$ for some faithful linear character $\lambda \in \operatorname{Irr}(Z)$, and that $Z=Z(X)$. First assume $g$ does not act trivially on $C$. Then there are some $x \in Q$ and $1 \neq z \in Z$ such that $x g x^{-1}=g z$, whence $\chi(g)=\chi(g) \lambda(z)$ and so $\chi(g)=0$. Next, assume $g$ acts trivially on $C$. Consider the alternating form $(x Z, y Z) \mapsto[x, y] \in Z$ for $x Z, y Z \in Q / Z$. Since this form is nondegenerate, one can check that the orthogonal complement $\bar{C}^{\perp}$ is exactly $[g, Q / Z]$. If $u \in Q$, then $C_{Q / Z}(g u)=\bar{C}$; moreover, $g u$ acts trivially on $C$ if and only if $u$ centralizes $C$, i.e. $u Z \in \bar{C}^{\perp}$. It follows that the coset $g Q$ contains exactly $|Z| \cdot\left|\bar{C}^{\perp}\right|$ elements $g u$ that act trivially on $C$. For each such element $g u$, we have already shown that $u Z \in \bar{C}^{\perp}=[g, Q / Z]$, therefore $g^{-1} v g v^{-1}=u z$ for some $v \in Q$ and $z \in Z$, i.e. $v g v^{-1}=g u z$, whence $|\chi(g u)|=$ $\mid \chi($ guz $)|=| \chi(g) \mid$. Thus $\sum_{x \in g Q}|\chi(x)|^{2}=\sum_{u Z \in \bar{C}^{\perp}}|\chi(g u)|^{2}=|Z| \cdot\left|\bar{C}^{\perp}\right| \cdot|\chi(g)|^{2}$. On the other hand, $\sum_{x \in g Q}|\chi(x)|^{2}=|Q|$ by [IS, Lemma (3.4)]. Consequently, $|\chi(g)|^{2}=|Q / Z| /\left|\bar{C}^{\perp}\right|=|\bar{C}|$, as stated.

\section{Local properties $\left(\mathcal{W}_{2}^{\varepsilon}\right)$ and Weil Representations}

First we make some observations about the structure and representations of $P_{j}=\operatorname{Stab}_{G}\left(\left\langle e_{1}, \ldots, e_{j}\right\rangle_{\mathbb{F}_{q}}\right)$ for $1 \leq j \leq n$. It is convenient to write elements of $P_{j}$ with respect to the basis

$$
\left(e_{1}, \ldots, e_{j}, e_{j+1}, \ldots, e_{n}, f_{j+1}, \ldots, f_{n}, f_{1}, \ldots, f_{j}\right) .
$$

For any $A \in M_{2 n-2 j, j}(q)$ and $C \in M_{j}(q)$, set $[A, C]:=\left(\begin{array}{ccc}I_{j} & { }^{t} A J_{n-j} & C \\ 0 & I_{2 n-2 j} & A \\ 0 & 0 & I_{j}\end{array}\right)$. Then

$$
Q_{j}=\left\{[A, C] \mid A \in M_{2 n-2 j, j}\left(\mathbb{F}_{q}\right), C \in M_{j}(q), C+{ }^{t} C+{ }^{t} A J_{n-j} A=0\right\}
$$

has order $q^{j(4 n-3 j+1) / 2}$. The multiplication in $Q_{j}$ is given by the formula

$$
[A, C] \cdot\left[A^{\prime}, C^{\prime}\right]=\left[A+A^{\prime}, C+C^{\prime}+{ }^{t} A J_{n-j} A^{\prime}\right] .
$$

In particular, $Z_{j}=\left\{[0, C] \mid C \in \mathcal{H}_{j}(q)\right\}$ and $Q_{j}^{\prime}=\left\{[0, C] \mid C \in \mathcal{H}_{j}^{0}(q)\right\}$ if $j>1$.

Assume $j>1$. Any linear character $\lambda$ of $Z_{j}$ (over $\mathbb{F}$ ) can be written in the form $\lambda_{B} \quad:[0, X] \mapsto(-1)^{\operatorname{tr}_{\mathbb{F}_{q} / \mathbb{F}_{2}}(\operatorname{Tr}(B X))}$ for some $B \in M_{j}(q)$. Clearly, $\lambda_{B}=\lambda_{B^{\prime}}$ if and only if $B-B^{\prime} \in \mathcal{H}_{j}^{0}(q)$, so we may think of the subscript $B$ of $\lambda_{B}$ as a coset in $\mathcal{F}_{j}(q)$. On the other hand, for each $B=\left(b_{i j}\right) \in M_{j}(q)$ we define the quadratic form $q_{B}$ on the space $\mathbb{F}_{q}^{j}=\left\langle f_{1}, \ldots, f_{j}\right\rangle_{\mathbb{F}_{q}}$ such that $q_{B}\left(f_{i}\right)=b_{i i}$ and the associated bilinear form has $B+{ }^{t} B$ as Gram matrix in the given basis. Again, $q_{B}=q_{B^{\prime}}$ if and only if $B-B^{\prime} \in \mathcal{H}_{j}^{0}(q)$, so we may think of the subscript $B$ of $q_{B}$ as a coset in $\mathcal{F}_{j}(q)$. Furthermore, for any $X \in G L_{j}(q), \lambda_{t}{ }_{B X}=\lambda_{B}$ if and only if ${ }^{t} X B X-B \in \mathcal{H}_{j}^{0}(q)$, 
i.e. ${ }^{t} X B X$ and $B$ have the same diagonal and ${ }^{t} X\left({ }^{t} B+B\right) X={ }^{t} B+B$, that is, $X \in O\left(q_{B}\right)$. Recall that the rank of $q_{B}$ is the codimension of $\operatorname{rad}\left(q_{B}\right)$ in $\mathbb{F}_{q}^{j}$. Then two quadratic forms on $\mathbb{F}_{q}^{j}$ are $G L_{j}(q)$-equivalent if and only if they have the same rank and the same type if the rank is even. Let $\mathcal{O}_{r}^{\epsilon}$ be the set of all $\lambda_{B}$ where $q_{B}$ has rank $r$ and type $\epsilon$. Here $\epsilon= \pm$ if $r$ is even and $\epsilon$ is void if $r$ is odd. To ease the notation in what follows, we will also use the symbol $\mathcal{O}_{r}^{ \pm}$for $\mathcal{O}_{r}$ when $r$ is odd. The above discussion yields the following statement:

Lemma 3.1. Assume $1<j \leq n$. Then the $P_{j}$-orbits on $\operatorname{IBr}_{\ell}\left(Z_{j}\right)$ are precisely $\mathcal{O}_{r}^{\epsilon}$ with $0 \leq r \leq j$. If $\lambda=\lambda_{B}$ belongs to $\mathcal{O}_{r}^{\epsilon}$, then $S_{t a b_{L_{j}}}\left(\lambda_{B}\right)$ is isomorphic to

$$
S p_{2 n-2 j}(q) \times O\left(q_{B}\right) \simeq S p_{2 n-2 j}(q) \times\left(\left[q^{r(j-r)}\right]:\left(G L_{j-r}(q) \times O_{r}^{\epsilon}(q)\right)\right) .
$$

Definition 3.2. Let $r$ be even, $2 \leq r \leq n, \varepsilon= \pm$.

(i) Let $r \leq j \leq n$. We say that an $\mathbb{F} P_{j}$-module $V$ has property $\left(\mathcal{W}_{r}^{\varepsilon}\right)$ if all $Z_{j}$-characters that occur in the module $\left.V\right|_{Z_{j}}$ belong to $\mathcal{O}_{r}^{\varepsilon} \cup\left(\bigcup_{0 \leq s \leq r-1, \alpha= \pm} \mathcal{O}_{s}^{\alpha}\right)$.

(ii) We say that an $\mathbb{F} G$-module $V$ has property $\left(\mathcal{W}_{r}^{\varepsilon}\right)$ if the $\mathbb{F} P_{j}$-module $V$ satisfies $\left(\mathcal{W}_{r}^{\varepsilon}\right)$ for some $j \geq r$.

Definition 3.3. Let $2 \leq r \leq n$.

(i) Let $r<j \leq n$. We say that an $\mathbb{F} P_{j}$-module $V$ has property $\left(\mathcal{W}_{r}\right)$ if all $Z_{j}$-characters that occur in the module $\left.V\right|_{Z_{j}}$ belong to $\bigcup_{0 \leq s \leq r, \alpha= \pm} \mathcal{O}_{s}^{\alpha}$.

(ii) We say that an $\mathbb{F} G$-module $V$ has property $\left(\mathcal{W}_{r}\right)$ if the $\mathbb{F} P_{j}$-module $V$ satisfies $\left(\mathcal{W}_{r}\right)$ for some $j>r$.

We say that a Brauer character $\varphi$ has property $\left(\mathcal{W}_{r}^{\varepsilon}\right)$, resp. $\left(\mathcal{W}_{r}\right)$, if the same holds for a module affording this character.

Lemma 3.4. Let $V$ be an $\mathbb{F} G$-module.

(i) Suppose that $r \geq 2$ is even and the $\mathbb{F} P_{j}$-module $V$ satisfies $\left(\mathcal{W}_{r}^{\varepsilon}\right)$ for some $j \geq r$. Then the $\mathbb{F} P_{m}$-module $V$ satisfies $\left(\mathcal{W}_{r}^{\varepsilon}\right)$ for every $m \geq r$.

(ii) Suppose that $r \geq 2$ and the $\mathbb{F} P_{j}$-module $V$ satisfies $\left(\mathcal{W}_{r}\right)$ for some $j>r$. Then the $\mathbb{F} P_{m}$-module $\bar{V}$ satisfies $\left(\mathcal{W}_{r}\right)$ for every $m>r$.

Proof. (i) We may assume that $P_{m}=\operatorname{Stab}_{G}\left(\left\langle e_{1}, \ldots, e_{m}\right\rangle_{\mathbb{F}_{q}}\right)$. Then

$$
Z_{m}=\operatorname{Stab}_{G}\left(e_{1}, \ldots, e_{n}, f_{m+1}, \ldots, f_{n}\right) .
$$

In particular, $Z_{1}<Z_{2}<\ldots<Z_{n}$.

First we show that the $\mathbb{F} P_{n}$-module $V$ satisfies $\left(\mathcal{W}_{r}^{\varepsilon}\right)$. Assume the contrary: $\left.V\right|_{Z_{n}}$ affords a character $\lambda_{B}$ lying outside of $\mathcal{O}_{r}^{\varepsilon} \cup\left(\bigcup_{0 \leq s \leq r-1, \alpha= \pm} \mathcal{O}_{s}^{\alpha}\right)$. Conjugating $\lambda_{B}$ using $L_{n}$ we may assume that $\operatorname{rad}\left(q_{B}\right)=\left\langle f_{s+1}, \ldots, f_{n}\right\rangle_{\mathbb{F}_{q}}$, where $s=\operatorname{rank}\left(q_{B}\right)$. Observe that either $s>r$, or $s=r$ but $q_{B}$ has type $-\varepsilon$. In either case we may choose $f_{1}, \ldots, f_{r}$ such that $q_{B}$ is nondegenerate and of type $-\varepsilon$ on $\left\langle f_{1}, \ldots, f_{r}\right\rangle_{\mathbb{F}_{q}}$. It follows that $q_{B}$ restricted to $\left\langle f_{1}, \ldots, f_{j}\right\rangle_{\mathbb{F}_{q}}$ either has rank $>r$ or has rank $r$ but not type $\varepsilon$. Hence, $\left.\lambda_{B}\right|_{Z_{j}}$ cannot belong to $\mathcal{O}_{r}^{\varepsilon} \cup\left(\bigcup_{0 \leq s \leq r-1, \alpha= \pm} \mathcal{O}_{s}^{\alpha}\right)$, a contradiction.

Now we may assume that for any $\lambda_{B}$ occurring in $\left.V\right|_{Z_{n}}$, either $\operatorname{rank}\left(q_{B}\right)<r$ or $\operatorname{rank}\left(q_{B}\right)=r$ but $q_{B}$ has type $\varepsilon$ (on $\left.\left\langle f_{1}, \ldots, f_{n}\right\rangle_{\mathbb{F}_{q}}\right)$. The same obviously holds for the restriction of $q_{B}$ to $\left\langle f_{1}, \ldots, f_{m}\right\rangle_{\mathbb{F}_{q}}$ for any $m \geq r$. Hence $\left.\lambda_{B}\right|_{Z_{m}}$ belongs to $\mathcal{O}_{r}^{\varepsilon} \cup\left(\bigcup_{0 \leq s \leq r-1, \alpha= \pm} \mathcal{O}_{s}^{\alpha}\right)$, as desired.

(ii) Similarly to (i). 
We will frequently use the following consequence of Lemma 3.4

Corollary 3.5. Let $G=S p_{2 n}(q)$ and let $H \simeq S p_{2 m}(q)$ be a standard subgroup of $G$, where $2 \leq m \leq n$. Assume $\varphi \in \operatorname{IBr}_{\ell}(G)$ and $\varepsilon= \pm$. Then $\varphi$ satisfies $\left(\mathcal{W}_{2}^{\varepsilon}\right)$ if and only if $\left(\mathcal{W}_{2}^{\varepsilon}\right)$ holds for $\left.\varphi\right|_{H}$ (equivalently, if $\left(\mathcal{W}_{2}^{\varepsilon}\right)$ holds for all irreducible constituents of $\left.\left.\varphi\right|_{H}\right)$.

Proof. We may assume that $H=\operatorname{Stab}_{G}\left(e_{m+1}, \ldots, e_{n}, f_{m+1}, \ldots, f_{n}\right)$. Consider the parabolic subgroup $P_{2}(H):=\operatorname{Stab}_{H}\left(\left\langle e_{1}, e_{2}\right\rangle_{\mathbb{F}_{q}}\right)$ of $H$. Then its subgroup $Z_{2}(H):=O_{2}\left(P_{2}(H)\right)$ is exactly the subgroup $Z_{2}$ for the parabolic subgroup $P_{2}=$ $\operatorname{Stab}_{G}\left(\left\langle e_{1}, e_{2}\right\rangle_{\mathbb{F}_{q}}\right)$ of $G$. By definition, $\varphi$ satisfies $\left(\mathcal{W}_{2}^{\varepsilon}\right)$ if and only if all $Z_{2}$-characters occuring in $\left.\varphi\right|_{Z_{2}}$ belong to $\mathcal{O}_{0} \cup \mathcal{O}_{1} \cup \mathcal{O}_{2}^{\varepsilon}$. Hence the statement follows.

The properties $\left(\mathcal{W}_{2}^{+}\right)$and $\left(\mathcal{W}_{2}^{-}\right)$will play a central role throughout the paper. It turns out that they are the local properties that distinguish low-dimensional representations from the rest. Unlike the case of $S L_{n}(q), S U_{n}(q)$ and the case of $S p_{2 n}(q)$ with $q$ odd, where Weil representations have degree $<q^{n}$ and they are the obvious candidates for low-dimensional representations, $G=S p_{2 n}(q)$ with $q$ even does not have any nontrivial representations of dimension less than $d(G):=$ $\left(q^{n}-1\right)\left(q^{n}-q\right) / 2(q+1)$ LaS]. However, we can still introduce two classes of irreducible complex representations of $G$, which come from Weil representations of $S L_{2 n}(q)$ and $S U_{2 n}(q)$ and which we will call Weil representations of $G$.

We briefly recall the definition of complex Weil representations of $S L_{2 n}(q)$. Consider the permutation character $\tau_{n}: g \mapsto\left|C_{M}(g)\right|=q^{\operatorname{dim}_{\mathbb{F}_{q}} \operatorname{Ker}(g-1)}$ of $S L_{2 n}(q)$ on the points of the natural module $M:=\mathbb{F}_{q}^{2 n}$. It decomposes into irreducible constituents as $2 \cdot 1+\sum_{i=0}^{q-2} \tau_{n}^{i}$, where

$$
\tau_{n}^{i}(g)=\frac{1}{q-1} \sum_{j=0}^{q-2} \tilde{\delta}^{i j} q^{\operatorname{dim}_{\mathbb{F}_{q}} \operatorname{Ker}\left(g-\delta^{j}\right)}-2 \delta_{i, 0}
$$

for any $g \in S L_{2 n}(q)$ (and $\operatorname{Ker}\left(g-\delta^{j}\right)$ is computed on $M$ ). The $\tau_{n}^{i}, 0 \leq i \leq q-2$, are the (irreducible) complex Weil representations of $S L_{2 n}(q)$ (regardless if $q$ is even or odd); cf. [Ge, Hw1. Formula (11) can be derived following the proof of TZ2, Lemma 4.1].

We can embed $G=S p_{2 n}(q)$ in $S L_{2 n}(q)$. Since the $G$-module $M$ is self-dual, $\operatorname{dim} \operatorname{Ker}\left(g-\delta^{j}\right)=\operatorname{dim} \operatorname{Ker}\left(g-\delta^{-j}\right)$ for any $g$ and $j$. It follows that

$$
\left.\tau_{n}^{i}\right|_{G}=\left.\tau_{n}^{q-1-i}\right|_{G} \text { for } 1 \leq i \leq q-2 .
$$

It is known, see for instance [ [T], that the characters $\left.\tau_{n}^{i}\right|_{G}$ with $1 \leq i \leq q / 2-1$ are distinct irreducible characters of $G$ (of degree $\left(q^{2 n}-1\right) /(q-1)$ ), and we denote by the same symbol $\tau_{n}^{i}$. On the other hand,

$$
\left.\tau_{n}^{0}\right|_{G}=\rho_{n}^{1}+\rho_{n}^{2}
$$

where $\rho_{n}^{1}$, resp. $\rho_{n}^{2}$, is an irreducible character of $G$ of degree $\left(q^{n}+1\right)\left(q^{n}-q\right) / 2(q-1)$, resp. $\left(q^{n}-1\right)\left(q^{n}+q\right) / 2(q-1)$.

Next we briefly recall the definition of complex Weil representations of $S U_{2 n}(q)$. Consider the class function $\zeta_{n}: g \mapsto(-q)^{\operatorname{dim}_{\mathbb{F}^{2}} \operatorname{Ker}(g-1)}$ of $S U_{2 n}(q)$ (where $\operatorname{Ker}(g-1)$ is the fixed point subspace of $g$ on the natural module $\widetilde{M}:=\mathbb{F}_{q^{2}}^{2 n}$. One can prove 
(cf. [TZ2]) that $\zeta_{n}$ decomposes into irreducible constituents as $\sum_{i=0}^{q} \zeta_{n}^{i}$, where

$$
\zeta_{n}^{i}(g)=\frac{1}{q+1} \sum_{j=0}^{q} \tilde{\xi}^{i j}(-q)^{\operatorname{dim}_{\mathbb{F}^{2}} \operatorname{Ker}\left(g-\xi^{j}\right)}
$$

for any $g \in S U_{2 n}(q)$ (and $\operatorname{Ker}\left(g-\xi^{j}\right)$ is calculated on $\widetilde{M}$ ). The $\zeta_{n}^{i}, 0 \leq i \leq q$ are the (irreducible) complex Weil representations of $S U_{2 n}(q)$ (regardless if $q$ is even or odd); cf. [Ge, [Hw1], [S].

We can identify $\widetilde{M}$ with $M \otimes_{\mathbb{F}_{q}} \mathbb{F}_{q^{2}}$ and embed $G=S p_{2 n}(q)$ in $S U_{2 n}(q)$ (cf. for instance $\mathrm{KLi}]$ ). Since the $G$-module $M$ is self-dual, it follows that $\operatorname{dim} \operatorname{Ker}\left(g-\xi^{j}\right)=$ $\operatorname{dim} \operatorname{Ker}\left(g-\xi^{-j}\right)$ for any $g$ and $j$. It follows that

$$
\left.\zeta_{n}^{i}\right|_{G}=\left.\zeta_{n}^{q+1-i}\right|_{G} \text { for } 1 \leq i \leq q .
$$

As shown in $[\mathrm{T}]$, the characters $\left.\zeta_{n}^{i}\right|_{G}$ with $1 \leq i \leq q / 2$ are distinct irreducible characters of $G$ (of degree $\left(q^{2 n}-1\right) /(q+1)$ ), and we denote by the same symbol $\zeta_{n}^{i}$. On the other hand,

$$
\left.\zeta_{n}^{0}\right|_{G}=\alpha_{n}+\beta_{n}
$$

where $\alpha_{n}$, resp. $\beta_{n}$, is an irreducible character of $G$ of degree $\left(q^{n}-1\right)\left(q^{n}-q\right) / 2(q+1)$, resp. $\left(q^{n}+1\right)\left(q^{n}+q\right) / 2(q+1)$. Observe that $\alpha_{n}(\bmod \ell)$ is irreducible, since its degree meets the Landazuri-Seitz-Zalesskii bound $d(G)$.

Definition 3.6. (i) The $q / 2+1$ characters $\rho_{n}^{1}, \rho_{n}^{2}$, and $\tau_{n}^{i}$ with $1 \leq i \leq q / 2-1$ are called (complex) linear-Weil characters of $G$. The nontrivial irreducible constituents of reductions modulo $\ell$ of these $q / 2+1$ characters are called linear-Weil characters of $G$ in characteristic $\ell$.

(ii) The $q / 2+2$ characters $\alpha_{n}, \beta_{n}$, and $\zeta_{n}^{i}$ with $1 \leq i \leq q / 2$ are called unitaryWeil characters of $G$. The nontrivial irreducible constituents of reductions modulo $\ell$ of these $q / 2+2$ characters are called unitary-Weil characters of $G$ in characteristic $\ell$.

(iii) In general, a Weil character of $G$ is either a linear-Weil or a unitary-Weil (Brauer) character.

Weil characters behave well when restricted to certain naturally embedded subgroups:

Lemma 3.7. Let $G=S p_{2 n}(q)$ and let $\rho$ be a Weil character of $G$. Let $H \simeq S p_{2 m}(q)$ be a standard subgroup of $G$, and let $K \simeq S p_{2 k}\left(q^{l}\right)$, where $k l=n$, be naturally embedded in $G$. Let $\phi$ be any nontrivial irreducible constituent of $\left.\rho\right|_{H}$ and let $\psi$ be any nontrivial irreducible constituent of $\left.\rho\right|_{K}$.

(i) Assume that $\rho$ is a linear-Weil character. Then $\phi$, resp. $\psi$, is also a linearWeil character.

(ii) Assume that $\rho$ is a unitary-Weil character. Then $\phi$ is also a unitary-Weil character. If $l$ is even, then $\psi$ is linear-Weil, and if $l$ is odd, then $\psi$ is unitary-Weil.

Proof. Clearly, $\left.\tau_{n}\right|_{H}=q^{2 n-2 m} \tau_{m}$ and $\left.\zeta_{n}\right|_{H}=q^{2 n-2 m} \zeta_{m}$, whence the statements follow for $\phi$. Next, consider the characters $\tau_{k, q^{l}} \quad: \quad g \mapsto\left(q^{l}\right)^{\operatorname{dim}_{q^{l}}} \operatorname{Ker}(g-1)$ and $\zeta_{k, q^{l}}: g \mapsto\left(-q^{l}\right)^{\operatorname{dim}_{\mathbb{F}^{2 l}} \operatorname{Ker}(g-1)}$ of $K$, which play the roles that $\tau_{n}$ and $\zeta_{n}$ play for $G$. Then $\left.\tau_{n}\right|_{K}=\tau_{k, q^{l}}$, and $\left.\zeta_{n}\right|_{K}=\tau_{k, q^{l}}$ if $l$ is even and $\zeta_{k, q^{l}}$ if $l$ is odd. Hence the statements follow for $\psi$. 
The following lemma is particularly useful in computing the values of Weil characters at unipotent elements:

Lemma 3.8. Let $g \in G=S p_{2 n}(q)$ be any element and $i>0$. If $(|g|, q-1)=1$, then $\tau_{n}^{i}(g)=\rho_{n}^{1}(g)+\rho_{n}^{2}(g)+1$. If $(|g|, q+1)=1$, then $\zeta_{n}^{i}(g)=\alpha_{n}(g)+\beta_{n}(g)-1$.

Proof. Assume $(|g|, q-1)=1$. Then $\operatorname{dim} \operatorname{Ker}\left(g-\delta^{j}\right)=0$ if $j>0$. By (1) it follows for any $k$ that

$$
\tau_{n}^{k}(g)=-2 \delta_{k, 0}+\frac{q^{\operatorname{dim} \operatorname{Ker}(g-1)}+\sum_{j=1}^{q-2} \tilde{\delta}^{j k}}{q-1}=-\delta_{k, 0}+\frac{q^{\operatorname{dim} \operatorname{Ker}(g-1)}-1}{q-1} .
$$

Using this formula for $k=0$ and for $k=i>0$, we get $\tau_{n}^{i}(g)-\tau_{n}^{0}(g)=1$, which yields the statement for $\tau_{n}^{i}(g)$ because of (3) . The proof for $\zeta_{n}^{i}(g)$ is similar.

\section{Cross Characteristic Representations of Small groups}

In this section we consider the groups $S p_{4}(q), S p_{6}(2)$, and $S p_{8}(2)$.

The character table of $S p_{4}(q)$ has been computed in $\mathrm{E}$, and we will keep the notation used there.

Proposition 4.1. Let $G=S p_{4}(q)$ and suppose that $\varphi \in \operatorname{IBr}_{\ell}(G)$ satisfies $\left(\mathcal{W}_{2}^{\varepsilon}\right)$ for some $\varepsilon= \pm$. Then one of the following holds:

(i) $\varphi$ is either trivial or a Weil character.

(ii) $q=2, \ell=3, \varepsilon=+$, and $\varphi$ is the unique irreducible 3 -Brauer character of degree 6 .

(iii) $q=2, \ell \neq 3, \varepsilon=+$, and $\varphi=\widehat{\chi_{12}(1)}$ (of degree 10). Conversely, if $\varphi$ satisfies one of $(\mathrm{i})-(\mathrm{iii})$, then $\varphi$ satisfies $\left(\mathcal{W}_{2}^{\varepsilon}\right)$ for some $\varepsilon= \pm$.

Proof. The Levi subgroup $L_{2}=G L_{2}(q)$ acts on $Z_{2}^{\#}$ with three orbits of length $q-1$, resp. $q^{2}-1,\left(q^{2}-1\right)(q-1)$, whose representatives belong to class $A_{31}$, resp. $A_{2}, A_{32}$, of $G$. On the other hand, $L_{2}$ acts on $\operatorname{IBr}_{\ell}\left(Z_{2}\right) \backslash\{1\}$ with three orbits, $\mathcal{O}_{1}$, $\mathcal{O}_{2}^{+}$, and $\mathcal{O}_{2}^{-}$. Define

$$
\omega_{1}=\sum_{\alpha \in \mathcal{O}_{1}} \alpha, \omega_{2}^{+}=\sum_{\alpha \in \mathcal{O}_{2}^{+}} \alpha, \omega_{2}^{-}=\sum_{\alpha \in \mathcal{O}_{2}^{-}} \alpha .
$$

It is easy to compute the character values of these three characters on $Q_{2}$ :

\begin{tabular}{|c|c|c|c|c|}
\hline & 1 & $A_{31}$ & $A_{2}$ & $A_{32}$ \\
\hline$\omega_{1}$ & $q^{2}-1$ & $q^{2}-1$ & -1 & -1 \\
\hline$\omega_{2}^{+}$ & $q\left(q^{2}-1\right) / 2$ & $-q(q+1) / 2$ & $q(q-1) / 2$ & $-q / 2$ \\
\hline$\omega_{2}^{-}$ & $q(q-1)^{2} / 2$ & $-q(q-1) / 2$ & $-q(q-1) / 2$ & $q / 2$ \\
\hline
\end{tabular}

Therefore, $\left.\chi\right|_{Z_{2}}=a(\chi) \cdot 1+b(\chi) \omega_{1}+c(\chi) \omega_{2}^{+}+d(\chi) \omega_{2}^{-}$, where

$$
\left\{\begin{array}{l}
c(\chi)=\left(\chi(1)+(q-1) \chi\left(A_{2}\right)-\chi\left(A_{31}\right)-(q-1) \chi\left(A_{32}\right)\right) / q^{3}, \\
d(\chi)=\left(\chi(1)-(q+1) \chi\left(A_{2}\right)-\chi\left(A_{31}\right)+(q+1) \chi\left(A_{32}\right)\right) / q^{3} .
\end{array}\right.
$$

Clearly, $\varphi$ satisfies $\left(\mathcal{W}_{2}^{+}\right)$if and only if $d(\varphi)=0$, and $\varphi$ satisfies $\left(\mathcal{W}_{2}^{-}\right)$if and only if $c(\varphi)=0$. Hence the proposition in the case of complex representations follows from direct calculation using $[\mathrm{E}]$. The irreducible Brauer characters of $G$ are also available from [Wh1, and direct calculation proves the proposition in the modular case. Observe that the character $\varphi$ mentioned in (ii) is $\widehat{\theta_{4}}+1-\widehat{\theta_{2}}-\widehat{\theta_{3}}-\widehat{\theta_{5}}$. 
For the converse, we apply (7) to the character $\tau_{2}$. If $g=\left(\begin{array}{cc}I_{2} & X \\ 0 & I_{2}\end{array}\right)$ is any element in $Z_{2}$, then $\tau_{2}(g)=q^{2+\operatorname{dim} \operatorname{Ker}(X)}$. It follows that $\tau_{2}$ takes value $q^{2}$, resp. $q^{3}, q^{2}$, on classes $A_{31}$, resp. $A_{2}, A_{32}$. Hence

$$
\left.\tau_{2}\right|_{Z_{2}}=\left(2 q^{2}-1\right) \cdot 1_{Z_{2}}+(q-1) \omega_{1}+(2 q-2) \omega_{2}^{+} .
$$

In particular, $\left(\mathcal{W}_{2}^{+}\right)$holds for $\tau_{2}$. Similarly,

$$
\left.\zeta_{2}\right|_{Z_{2}}=1_{Z_{2}}+(q+1) \omega_{1}+(2 q+2) \omega_{2}^{-} .
$$

In particular, $\left(\mathcal{W}_{2}^{-}\right)$holds for $\zeta_{2}$. Now if $\varphi$ is trivial or a Weil character, then by definition $\varphi$ is a constituent of $\widehat{\tau_{2}}$ or $\widehat{\zeta_{2}}$, and therefore $\varphi$ satisfies $\left(\mathcal{W}_{2}^{\varepsilon}\right)$ for some $\varepsilon$.

Corollary 4.2. Let $\varphi \in \operatorname{IBr}_{\ell}\left(S p_{2 n}(q)\right)$ with $n \geq 2$. If $\varphi$ is a linear-Weil character, then $\varphi$ satisfies $\left(\mathcal{W}_{2}^{+}\right)$. If $\varphi$ is a unitary-Weil character, then $\varphi$ satisfies $\left(\mathcal{W}_{2}^{-}\right)$.

Proof. Let $G=S p_{2 n}(q)$ and consider $H=\operatorname{Stab}_{G}\left(e_{3}, \ldots, e_{n}, f_{3}, \ldots, f_{n}\right)$. Then $H \simeq$ $S p_{4}(q)$. Moreover, if we consider the parabolic subgroup $P_{2}(H):=S t a b_{H}\left(\left\langle e_{1}, e_{2}\right\rangle_{\mathbb{F}_{q}}\right)$ of $H$, then its subgroup $Z_{2}(H):=O_{2}\left(P_{2}(H)\right)$ is exactly the subgroup $Z_{2}$ for the parabolic subgroup $P_{2}=S t a b_{G}\left(\left\langle e_{1}, e_{2}\right\rangle_{\mathbb{F}_{q}}\right)$ of $G$. Observe that $\left.\tau_{n}\right|_{Z_{2}}=\left.q^{2 n-4} \cdot \tau_{2}\right|_{Z_{2}}$, and $\left.\zeta_{n}\right|_{Z_{2}}=\left.q^{2 n-4} \cdot \zeta_{2}\right|_{Z_{2}}$. Hence $\left(\mathcal{W}_{2}^{+}\right)$holds for $\tau_{n}$ by (88), and $\left(\mathcal{W}_{2}^{-}\right)$holds for $\zeta_{n}$ by (9).

One of the main goals of the paper is to prove that the converse of Corollary 4.2 is also true (except for $(n, q)=(2,2)$ and $(3,2))$.

Lemma 4.3. Let $G=S p_{6}(2)$ or $S p_{8}(2)$, and $\varphi \in \operatorname{IBr}_{\ell}(G)$. Then $\varphi$ satisfies $\left(\mathcal{W}_{2}^{\varepsilon}\right)$ for some $\varepsilon= \pm$ if and only if one of the following holds:

(i) $\varphi$ is either trivial or a Weil character.

(ii) $G=S p_{6}(2), \varepsilon=+$ and $\varphi=\widehat{\chi_{4}}$ (where $\chi_{4}$ is an irreducible complex character of $G$ of degree 21 in the notation of [Atlas]).

Proof. Consider a standard subgroup $H \simeq S p_{4}(2)$. Then elements of class $A_{2}$, resp. $A_{31}, A_{32}$, of $H$ (in the notation of [E]) belong to class $2 A$, resp. $2 B, 2 C$, of $G$ (in the notation of [Atlas]). By Corollary [3.5, $\varphi$ satisfies $\left(\mathcal{W}_{2}^{+}\right)$if and only if $\left(\mathcal{W}_{2}^{+}\right)$ holds for $\left.\varphi\right|_{H}$, which is, by the virtue of (7), equivalent to

$$
\chi(1)-3 \chi(2 A)-\chi(2 B)+3 \chi(2 C)=0 .
$$

Similarly, $\varphi$ satisfies $\left(\mathcal{W}_{2}^{-}\right)$if and only if

$$
\chi(1)+\chi(2 A)-\chi(2 B)-\chi(2 C)=0 .
$$

Now the lemma follows from direct calculation using character values of $G$ given in Atlas and [JLPW]. (Note that in the case $G=S p_{8}(2)$, Proposition 5.7 (below) implies that $\varphi$ can satisfy $\left(\mathcal{W}_{2}^{\varepsilon}\right)$ only when for any nontrivial $Q_{1}$-character $\lambda$ occurring in $\left.\varphi\right|_{Q_{1}}$, the inertia group $\operatorname{Stab}_{P_{1}}(\lambda)$ fixes a subspace of dimension 1 on the corresponding homogeneous component, and hence $\varphi(1) \leq 9180$. Thus it suffices to check the irreducible Brauer characters of degree $\leq 9180$.) 


\section{THE LOCAL PROPERTY $\left(\mathcal{W}_{1}\right)$}

Now we turn our attention to $Q_{1}$. Clearly, $Q_{1}$ is elementary abelian. Next, $L_{1}=L_{1}^{\prime} \times T_{1}$, where $L_{1}^{\prime} \simeq S p_{2 n-2}(q)$ and $T_{1} \simeq \mathbb{Z}_{q-1}$. We will write elements of $P_{1}=\operatorname{Stab}_{G}\left(\left\langle e_{1}\right\rangle_{\mathbb{F}_{q}}\right)$ with respect to the basis $\left(e_{1}, e_{2}, \ldots, e_{n}, f_{2}, \ldots, f_{n}, f_{1}\right)$. The conjugation by $\operatorname{diag}(1, X, 1)$, where $X \in S p_{2 n-2}(q)$, sends any $[A, C] \in Q_{1}$ to $[X A, C]$, and the conjugation by $\boldsymbol{c}_{\alpha}:=\operatorname{diag}\left(\alpha, I_{2 n-2}, \alpha^{-1}\right) \in T_{1}$, where $\alpha \in \mathbb{F}_{q}^{\bullet}$, sends $[A, C]$ to $\left[\alpha A, \alpha^{2} C\right]$. The map $[A, C] \mapsto C$ defines an $L_{1}^{\prime}$-invariant nondegenerate quadratic form on $Q_{1} \simeq \mathbb{F}_{q}^{2 n-1}$. We recall the following

Lemma 5.1 ([LaS, Lemma 2.2]). Let $V$ be an $n$-dimensional vector space over a field $\mathbb{F}_{q}, q=p^{f}$, and let $\mathbb{F}$ be an algebraically closed field of characteristic other than $p$. If $\lambda$ is a nontrivial linear character of (the additive group) $V$ over $\mathbb{F}$, then $\operatorname{Ker}(\lambda)$ contains a unique hyperplane of $V$.

Lemma 5.1 implies that $P_{1}$ acts on $\operatorname{IBr}_{\ell}\left(Q_{1}\right)$ with four orbits: $\left\{1_{Q_{1}}\right\}, \mathcal{O}_{1}$ of length $q^{2 n-2}-1$, and $\mathcal{O}_{2}^{\varepsilon}$ of length $q^{n-1}\left(q^{n-1}+\varepsilon\right)(q-1) / 2$ for $\varepsilon= \pm$. Here $\mathcal{O}_{1}$ is the set of nontrivial characters that are trivial on $Z_{1}=Z\left(P_{1}^{\prime}\right)$, and $\mathcal{O}_{2}^{\varepsilon}$ consists of nontrivial characters that are trivial on a hyperplane of type $\varepsilon$ (with respect to the above quadratic form). Denote $K_{\lambda}:=\operatorname{Stab}_{P_{1}}(\lambda), I_{\lambda}:=\operatorname{Stab}_{L_{1}}(\lambda), J_{\lambda}:=\operatorname{Stab}_{L_{1}^{\prime}}(\lambda)$. In particular, $I_{\lambda}=J_{\lambda} \simeq O_{2 n-2}^{\varepsilon}(q)$ for any $\lambda \in \mathcal{O}_{2}^{\varepsilon}$.

Definition 5.2. Let $V$ be any $\mathbb{F} G$-module. We say that $V$ has property $\left(\mathcal{W}_{1}\right)$ if for any nontrivial linear character $\lambda$ occuring in $\left.V\right|_{Q_{1}}$, all $I_{\lambda}$-composition factors on the $\lambda$-homogeneous component $V_{\lambda}$ of $V$ are of dimension 1 .

Even though property $\left(\mathcal{W}_{2}^{\varepsilon}\right)$ is more transparent than $\left(\mathcal{W}_{1}\right)$, it turns out that it is more convenient to work with $\left(\mathcal{W}_{1}\right)$ while classifying low-dimensional representations. In order to clarify the relationship between $\left(\mathcal{W}_{2}^{\varepsilon}\right)$ and $\left(\mathcal{W}_{1}\right)$ we need some auxiliary statements.

Lemma 5.3. Let $\chi$ be any Brauer character of a subgroup $I$ of a finite group $X$. Then for any subgroup $Y$ of $X,\left.\operatorname{Ind}_{I}^{X}(\chi)\right|_{Y}$ contains the Brauer character $\operatorname{Ind}_{Y \cap I}^{Y}\left(\left.\chi\right|_{Y \cap I}\right)$.

Proof. By Mackey's formula, $\left.\operatorname{Ind}_{I}^{X}(\chi)\right|_{Y}=\sum_{t \in Y \backslash X / I} \operatorname{Ind}_{Y \cap I^{t}}^{Y}\left(\left.\chi^{t}\right|_{Y \cap I^{t}}\right)$, where $I^{t}=$ $t I t^{-1}$ and $\chi^{t}(x)=\chi\left(t^{-1} x t\right)$. Hence the statement follows.

Lemma 5.4. Consider the subgroup $Z_{2}$ of $H=S p_{4}(q)$.

(i) Let $A=\left\{[a]_{1}:=\left(\begin{array}{cccc}1 & 0 & a & 0 \\ 0 & 1 & 0 & 0 \\ 0 & 0 & 1 & 0 \\ 0 & 0 & 0 & 1\end{array}\right) \mid a \in \mathbb{F}_{q}\right\}$. If $\nu$ is any $\mathbb{F} A$-character which is not trivial on $A$, then $\operatorname{Ind}_{A}^{Z_{2}}(\nu)$ intersects all three orbits $\mathcal{O}_{1}, \mathcal{O}_{2}^{+}$, and $\mathcal{O}_{2}^{-}$of $Z_{2}$-characters.

(ii) Let $A=\left\{[a]_{2}:=\left(\begin{array}{llll}1 & 0 & 0 & a \\ 0 & 1 & a & 0 \\ 0 & 0 & 1 & 0 \\ 0 & 0 & 0 & 1\end{array}\right) \mid a \in \mathbb{F}_{q}\right\}$. If $\nu$ is any $\mathbb{F} A$-character which is not trivial on $A$, then $\operatorname{Ind}_{A}^{Z_{2}}(\nu)$ intersects both orbits $\mathcal{O}_{2}^{+}$and $\mathcal{O}_{2}^{-}$of $Z_{2}$-characters. 
(iii) Let $A=\left\{[a, b]_{3}:=\left(\begin{array}{cccc}1 & 0 & a & b \\ 0 & 1 & b & b^{2} \\ 0 & 0 & 1 & 0 \\ 0 & 0 & 0 & 1\end{array}\right) \mid a=0,1, b \in \mathbb{F}_{q}\right\}$. Suppose that $\nu$ is any $\mathbb{F} A$-character whose generalized kernel does not contain $[1,0]_{3}$ and $[0,1]_{3}$. Then $\operatorname{Ind}_{A}^{Z_{2}}(\nu)$ intersects the orbit $\mathcal{O}_{2}^{+}$of $Z_{2}$-characters. If $q \geq 4$, then $\operatorname{Ind}_{A}^{Z_{2}}(\nu)$ also intersects $\mathcal{O}_{2}^{-}$.

Proof. (i) Since $\nu$ is not trivial on $A, \nu$ contains a nontrivial $A$-character $\mu:[a]_{1} \mapsto$ $(-1)^{\operatorname{tr}_{\mathbb{F}_{q} / \mathbb{F}_{2}}(a \alpha)}$ for some $\alpha \in \mathbb{F}_{q}^{\bullet}$. Choose $\beta, \gamma \in \mathbb{F}_{q}$ such that the polynomial $\alpha t^{2}+$ $\gamma t+\beta$ is irreducible over $\mathbb{F}_{q}$. Consider the $Z_{2}$-characters $\lambda_{i}=\lambda_{B_{i}}$ for $i=1,2,3$, where $B_{1}=\left(\begin{array}{cc}\alpha & 0 \\ 0 & 0\end{array}\right), B_{2}=\left(\begin{array}{cc}\alpha & 1 \\ 0 & 0\end{array}\right), B_{3}=\left(\begin{array}{cc}\alpha & \gamma \\ 0 & \beta\end{array}\right)$. Clearly, $\left.\lambda_{i}\right|_{A}=\mu$, whence $\operatorname{Ind}_{A}^{Z_{2}}(\nu)$ contains $\lambda_{i}$ for $i=1,2,3$. It remains to observe that $\lambda_{1} \in \mathcal{O}_{1}, \lambda_{2} \in \mathcal{O}_{2}^{+}$, and $\lambda_{3} \in \mathcal{O}_{2}^{-}$.

(ii) Since $\nu$ is not trivial on $A, \nu$ contains a nontrivial $A$-character $\mu:[a]_{2} \mapsto$ $(-1)^{\operatorname{tr}_{\mathbb{F}_{q} / \mathbb{F}_{2}}(a \gamma)}$ for some $\gamma \in \mathbb{F}_{q}^{\bullet}$. Choose $\alpha, \beta \in \mathbb{F}_{q}$ such that the polynomial $\alpha t^{2}+$ $\gamma t+\beta$ is irreducible over $\mathbb{F}_{q}$. Consider the $Z_{2}$-characters $\lambda_{i}=\lambda_{B_{i}}$ for $i=1,2$, where $B_{1}=\left(\begin{array}{cc}\alpha & \gamma \\ 0 & 0\end{array}\right), B_{2}=\left(\begin{array}{cc}\alpha & \gamma \\ 0 & \beta\end{array}\right)$. Clearly, $\left.\lambda_{i}\right|_{A}=\mu$, whence $\operatorname{Ind}_{A}^{Z_{2}}(\nu)$ contains $\lambda_{i}$ for $i=1,2$. It remains to observe that $\lambda_{1} \in \mathcal{O}_{2}^{+}$and $\lambda_{2} \in \mathcal{O}_{2}^{-}$.

(iii) Since the generalizer kernel of $\nu$ does not contain $[1,0]_{3}, \nu$ contains an $A$ character $\mu:[a, b]_{3} \mapsto(-1)^{\operatorname{tr}_{\mathbb{F}_{q} / \mathbb{F}_{2}}(b \gamma)}$ for some $\gamma \in \mathbb{F}_{q}$. Consider the $Z_{2}$-character $\lambda_{B}$, where $B=\left(\begin{array}{cc}0 & 1 \\ 0 & \gamma^{2}+1\end{array}\right)$. Clearly, $\left.\lambda_{B}\right|_{A}=\mu$, whence $\operatorname{Ind}_{A}^{Z_{2}}(\nu)$ contains $\lambda_{B}$, and $\lambda_{B} \in \mathcal{O}_{2}^{+}$.

Next assume that $q \geq 4$. Since the generalized kernel of $\nu$ does not contain $[0,1]_{3}, \nu$ contains an $A$-character $\mu^{\prime}:[a, b]_{3} \mapsto(-1)^{a \alpha+\operatorname{tr}_{\mathbb{F}_{q} / \mathbb{F}_{2}}(b \beta)}$ for some $\alpha=0,1$ and $\beta \in \mathbb{F}_{q}^{\bullet}$. Since $q \geq 4$, there is an $x \in \mathbb{F}_{q}^{\bullet}$ such that $\operatorname{tr}_{\mathbb{F}_{q} / \mathbb{F}_{2}}(x)=\alpha$. Let $V_{2}=\left\{t+t^{2} \mid t \in \mathbb{F}_{q}\right\}$. Since $\left|V_{2}\right|=q / 2 \leq q-2$, there is a $y_{0} \in \mathbb{F}_{q}^{\bullet} \backslash\left(1+x^{-1} V_{2}\right)$. Let $y=\beta y_{0}^{-1 / 2} \in \mathbb{F}_{q}^{\bullet}$. Then $\beta^{2}=y^{2} y_{0} \notin y^{2}+y^{2} x^{-1} V_{2}$. Finally, let $z=\beta^{2}-y^{2}$ and $C=\left(\begin{array}{ll}x & y \\ 0 & z\end{array}\right)$. The choice of $x, y, z$ ensures that $\left.\lambda_{C}\right|_{A}=\mu^{\prime}$, whence $\operatorname{Ind}_{A}^{Z_{2}}(\nu)$ contains $\lambda_{C}$. Claim that $\lambda_{C} \in \mathcal{O}_{2}^{-}$. Assume the contrary. Then there is some $s \in \mathbb{F}_{q}$ such that $x s^{2}+y s+z=0$. It follows that $x y^{-2}\left(\beta^{2}-y^{2}\right)=t^{2}+t \in V_{2}$ for $t=x s y^{-1}$, i.e. $\beta^{2} \in y^{2}+y^{2} x^{-1} V_{2}$, a contradiction.

Lemma 5.5. Consider the subgroup

$$
Z_{4}=O_{2}\left(\operatorname{Stab}_{H}\left(\left\langle e_{1}, \ldots, e_{4}\right\rangle_{\mathbb{F}_{q}}\right)\right)=\left\{[X]_{4}:=\left(\begin{array}{cc}
I_{4} & X \\
0 & I_{4}
\end{array}\right) \mid X \in \mathcal{H}_{4}(q)\right\}
$$

of $H=S p_{8}(q)$, and let $A=\operatorname{Stab}_{Z_{4}}\left(f_{4}\right)$. Suppose that $\nu$ is any $\mathbb{F} A$-character such that $\operatorname{Ker}(\nu) \nsupseteq A_{0}$, where $A_{0}=\left\{[X]_{4} \in A \mid X \in \mathcal{H}_{4}^{0}(q)\right\}$. Then $\operatorname{Ind}_{A}^{Z_{4}}(\nu)$ intersects at least one of the two orbits $\mathcal{O}_{4}^{+}$and $\mathcal{O}_{4}^{-}$of $Z_{4}$-characters.

Proof. Clearly, any linear character of $A$ is of the form $\mu_{C}:[X]_{4} \mapsto(-1)^{\operatorname{tr}_{\mathbb{F}_{q} / \mathbb{F}_{2}}(\operatorname{Tr}(C X))}$ for some $C \in M_{4}(q)$ with the last column and the last row equal to 0 . We may also consider the corresponding quadratic form $q_{C}$ on $\left\langle f_{1}, \ldots, f_{4}\right\rangle_{\mathbb{F}_{q}}$. Observe that if $\operatorname{rank}\left(q_{C}\right) \leq 1$, then $C$ is diagonal and so $\operatorname{Ker}\left(\mu_{C}\right) \geq A_{0}$. Hence our assumption 
on $\nu$ implies that $\nu$ contains a character $\mu_{C}$ with $\operatorname{rank}\left(q_{C}\right) \geq 2$. We may choose $f_{3}$ such that $f_{3}$ is contained in the radical of the bilinear form associated to $q_{C}$. Also, $\mu_{C}=\mu_{C^{\prime}}$ whenever $C-C^{\prime}$ belongs to $\mathcal{H}_{4}^{0}(q)$ and has the last column and the last row equal to 0 . Hence we may assume that $C=\left(\begin{array}{llll}a & b & 0 & 0 \\ c & d & 0 & 0 \\ 0 & 0 & e & 0 \\ 0 & 0 & 0 & 0\end{array}\right)$ for some $a, b, c, d, e \in \mathbb{F}_{q}$ and $b \neq c$. Define $B=\left(\begin{array}{cccc}a & b & 0 & 0 \\ c & d & 0 & 0 \\ 0 & 0 & e & 1 \\ 0 & 0 & 0 & 0\end{array}\right)$. Then $\left.\lambda_{B}\right|_{A}=\mu_{C}$, whence $\operatorname{Ind}_{A}^{Z_{4}}(\nu)$ contains $\lambda_{B}$. It remains to observe that $\operatorname{rank}\left(q_{B}\right)=4$.

Lemma 5.6. Consider the subgroup

$$
Z_{3}=O_{2}\left(\operatorname{Stab}_{H}\left(\left\langle e_{1}, e_{2}, e_{3}\right\rangle_{\mathbb{F}_{q}}\right)\right)=\left\{[X]_{5}:=\left(\begin{array}{cc}
I_{3} & X \\
0 & I_{3}
\end{array}\right) \mid X \in \mathcal{H}_{3}(q)\right\}
$$

of $H=S p_{6}(q)$, and let $A=S t a b_{Z_{3}}\left(f_{3}\right)$. Suppose that $\nu$ is any $\mathbb{F} A$-character such that $\operatorname{Ker}(\nu) \nsupseteq A_{0}$, where $A_{0}=\operatorname{Stab}_{Z_{3}}\left(f_{1}, f_{3}\right)$. Then $\operatorname{Ind}_{A}^{Z_{3}}(\nu)$ intersects the orbit $\mathcal{O}_{3}$ of $Z_{3}$-characters.

Proof. We assume that $\nu$ contains the $A$-character $\mu_{C}:[X]_{5} \mapsto(-1)^{\operatorname{tr}_{\mathbb{F}_{q} / \mathbb{F}_{2}}(\operatorname{Tr}(C X))}$ for some $C=\left(\begin{array}{lll}a & b & 0 \\ c & d & 0 \\ 0 & 0 & 0\end{array}\right)$, with $A_{0} \not \leq \operatorname{Ker}\left(\mu_{C}\right)$. The last condition means that $d \neq 0$. We may also consider the corresponding quadratic form $q_{C}$ on $\left\langle f_{1}, f_{2}, f_{3}\right\rangle_{\mathbb{F}_{q}}$. If $\operatorname{rank}\left(q_{C}\right)=2$, i.e. $b \neq c$, then define $B:=\left(\begin{array}{lll}a & b & 0 \\ c & d & 0 \\ 0 & 0 & 1\end{array}\right)$. If $\operatorname{rank}\left(q_{C}\right)=1$, i.e. $b=c$, then define $B:=\left(\begin{array}{lll}a & 0 & 1 \\ 0 & d & 0 \\ 0 & 0 & 0\end{array}\right)$. In both cases, $\operatorname{rank}\left(q_{B}\right)=3$, and $\left.\lambda_{B}\right|_{A}=\mu_{C}$, whence $\operatorname{Ind}_{A}^{Z_{3}}(\nu)$ contains $\lambda_{B}$.

Proposition 5.7. Let $G=S p_{2 n}(q)$ with $n \geq 3$ and $(n, q) \neq(3,2)$. Suppose that an $\mathbb{F} G$-module $V$ satisfies $\left(\mathcal{W}_{2}^{\varepsilon}\right)$ for some $\varepsilon= \pm$. Then $V$ also satisfies $\left(\mathcal{W}_{1}\right)$.

Proof. Assume the contrary. Then there is a nontrivial linear character $\lambda$ of $Q_{1}$ such that $I_{\lambda}:=\operatorname{Stab}_{L_{1}}(\lambda)$ has a composition factor of degree $>1$ on $V_{\lambda}$, the $\lambda$-homogeneous component of $V$. Clearly, $V$ contains the $P_{1}$-submodule $V^{\prime}:=$ $\sum_{\lambda^{\prime} \in \mathcal{O}} V_{\lambda^{\prime}}$ and $V^{\prime}=\operatorname{Ind}_{K_{\lambda}}^{P_{1}}\left(V_{\lambda}\right)$, where $\mathcal{O}$ is the orbit containing $\lambda$ and $K_{\lambda}=$ $\operatorname{Stab}_{P_{1}}(\lambda)$. Consider the standard subgroup $H=\operatorname{Stab}_{G}\left(e_{3}, \ldots, e_{n}, f_{3}, \ldots, f_{n}\right) \simeq$ $S p_{4}(q)$ of $G$ and the subgroup $Z_{2}=\operatorname{Stab}_{H}\left(e_{1}, e_{2}\right)$ of $H$. Let $K$ denote the kernel of $K_{\lambda}$ on $V_{\lambda}$.

1) First we assume that $\lambda \in \mathcal{O}_{1}$. We will assume that $P_{1}^{\prime}=\operatorname{Stab}_{G}\left(e_{3}\right)$. Then we may identify $K_{\lambda} \cap P_{1}^{\prime}$ with $\operatorname{Stab}_{G}\left(e_{3}, f_{2}+\left\langle e_{3}\right\rangle_{\mathbb{F}_{q}}\right)$. In this case $K_{\lambda} \cap Z_{2}=\operatorname{Stab}_{Z_{2}}\left(f_{2}\right)$ is the subgroup $A$ defined in Lemma5.4(i). Assume $\left.V_{\lambda}\right|_{A}$ contains a nontrivial linear character $\nu$. By Lemma 5.3, $\left.V^{\prime}\right|_{Z_{2}}$ contains $\operatorname{Ind}_{A}^{Z_{2}}(\nu)\left(\right.$ as $\left.Z_{2}<P_{1}^{\prime}\right)$, and $\operatorname{Ind}_{A}^{Z_{2}}(\nu)$ intersects both $\mathcal{O}_{2}^{+}$and $\mathcal{O}_{2}^{-}$by Lemma $5.4(\mathrm{i})$. It follows that $V$ cannot satisfy $\left(\mathcal{W}_{2}^{\varepsilon}\right)$, a contradiction. Therefore $A$ acts trivially on $V_{\lambda}$. 
We have shown that $K \geq A$. Observe that

$$
K_{\lambda} \cap P_{1}^{\prime}=Q_{1}: J_{\lambda}
$$

where $J_{\lambda}:=\operatorname{Stab}_{L_{1}^{\prime}}(\lambda)=\operatorname{Stab}_{G}\left(e_{3}, f_{3}, f_{2}\right)$ plays the role of the subgroup $P_{1}^{\prime}$ in the standard subgroup $\operatorname{Stab}_{G}\left(e_{3}, f_{3}\right) \simeq S p_{2 n-2}(q)$. Next, $J_{\lambda}=\left[q^{2 n-3}\right]: S$, where $S=\operatorname{Stab}_{G}\left(e_{2}, e_{3}, f_{2}, f_{3}\right) \simeq S p_{2 n-4}(q)$. Clearly, $A<K \cap S$. Thus $K \cap S$ is a normal subgroup of $S \simeq S p_{2 n-4}(q)$ that contains a long root subgroup $A$. It follows that $K \geq S$. Now $K \cap J_{\lambda}$ is a normal subgroup of $J_{\lambda}=\left[q^{2 n-3}\right]: S p_{2 n-4}(q)$ that contains $S p_{2 n-4}(q)$. Since $n \geq 3$, this implies that $K \geq J_{\lambda}$. Observe that $Q_{1}$ acts on $V_{\lambda}$ as scalars \pm 1 , and $I_{\lambda} / J_{\lambda} \simeq \mathbb{Z}_{q-1}$. It follows that all composition factors of the $I_{\lambda}$-module $V_{\lambda}$ are of dimension 1 , a contradiction.

2) Next we assume that $\lambda \in \mathcal{O}_{2}^{\alpha}$ for some $\alpha= \pm$. We will assume that $P_{1}=S t a b_{G}\left(\left\langle e_{n}\right\rangle_{\mathbb{F}_{q}}\right)$. In this case $I_{\lambda}=O(Q)<L_{1}^{\prime}=S t a b_{G}\left(e_{n}, f_{n}\right)$, where $Q$ is a quadratic form of type $\alpha$ on $\left\langle e_{1}, \ldots, e_{n-1}, f_{1}, \ldots, f_{n-1}\right\rangle_{\mathbb{F}_{q}}$ (and the associated bilinear form is $(\cdot, \cdot))$.

Assume $n \geq 4$ or $(n, \alpha)=(3,+)$. Then we may choose $e_{1}, e_{2}, f_{1}, f_{2}$ such that $Q$ is totally singular on $\left\langle e_{1}, e_{2}\right\rangle_{\mathbb{F}_{q}}$. In this case, $K_{\lambda} \cap Z_{2}$ is the subgroup $A$ defined in Lemma 5.4(ii). Assume $\left.V_{\lambda}\right|_{A}$ contains a nontrivial linear character $\nu$. By Lemma 5.3, $\left.V^{\prime}\right|_{Z_{2}}$ contains $\operatorname{Ind}_{A}^{Z_{2}}(\nu)\left(\right.$ as $\left.Z_{2}<P_{1}^{\prime}\right)$, and $\operatorname{Ind}_{A}^{Z_{2}}(\nu)$ intersects both $\mathcal{O}_{2}^{+}$and $\mathcal{O}_{2}^{-}$ by Lemma 5.4(ii). It follows that $V$ cannot satisfy $\left(\mathcal{W}_{2}^{\varepsilon}\right)$, a contradiction. Therefore $A$ acts trivially on $V_{\lambda}$. We have shown that $K \cap I_{\lambda}$ contains the subgroup $A$ of order $q$. Recall that $I_{\lambda}=O_{2 n-2}^{\alpha}(q)=\Omega_{2 n-2}^{\alpha}(q): 2$. We claim that $K \geq \Omega_{2 n-2}^{\alpha}(q)$. Indeed, if $n \geq 4$, then $\Omega_{2 n-2}^{\alpha}(q)$ is simple and it is the unique proper nontrivial normal subgroup of $I_{\lambda}$, whence $K \geq \Omega_{2 n-2}^{\alpha}(q)$. Assume $(n, \alpha)=(3,+)$. Since $(n, q) \neq(3,2)$, we have $q \geq 4$. Now $K \cap \Omega_{4}^{+}(q)$ contains the subgroup $A \cap \Omega_{4}^{+}(q)$ of order at least 2 , and $\Omega_{4}^{+}(q)=S_{1} \times S_{2}$ with $S_{i} \simeq S L_{2}(q)$, whence $K \cap \Omega_{4}^{+}(q)$ is either $S_{1}, S_{2}$, or $S_{1} \times S_{2}$. But any element from $O_{4}^{+}(q) \backslash \Omega_{4}^{+}(q)$ interchanges $S_{1}$ and $S_{2}$, hence $K \geq \Omega_{4}^{+}(q)$ as stated. Now it is clear that all composition factors of the $I_{\lambda}$-module $V_{\lambda}$ are of dimension 1 , again a contradiction.

Assume that $(n, \alpha)=(3,-)$. Then we may choose $e_{1}, e_{2}, f_{1}, f_{2}$ such that $Q$ has type - , resp. + , when restricted to $\left\langle e_{1}, f_{1}\right\rangle_{\mathbb{F}_{q}}$, resp. $\left\langle e_{2}, f_{2}\right\rangle_{\mathbb{F}_{q}}$, and moreover $Q\left(e_{1}\right)=1$ and $Q\left(e_{2}\right)=Q\left(f_{2}\right)=0$. In this case, $K_{\lambda} \cap Z_{2}$ is the subgroup $A$ defined in Lemma 5.4(iii). Let $K^{*}$ be the generalized kernel of $K_{\lambda}$ on $V_{\lambda}$. Assume $K^{*} \cap A=1$. By Lemma $\left.5.3 V^{\prime}\right|_{Z_{2}}$ contains $\operatorname{Ind}_{A}^{Z_{2}}\left(V_{\lambda}\right)\left(\right.$ as $\left.Z_{2}<P_{1}^{\prime}\right)$, and $\operatorname{Ind}_{A}^{Z_{2}}\left(V_{\lambda}\right)$ intersects both $\mathcal{O}_{2}^{+}$and $\mathcal{O}_{2}^{-}$by Lemma 5.4(iii) since $q \geq 4$. It follows that $V$ cannot satisfy $\left(\mathcal{W}_{2}^{\varepsilon}\right)$, a contradiction. Hence $K^{*} \cap A \neq 1$. Observe that $S L_{2}\left(q^{2}\right)$ is the unique proper nontrivial normal subgroup of $I_{\lambda}=O_{4}^{-}(q) \simeq S L_{2}\left(q^{2}\right): 2$, whence $K^{*} \geq S L_{2}\left(q^{2}\right), K \geq\left[K^{*}, K^{*}\right] \geq S L_{2}\left(q^{2}\right)$. It now follows that all composition factors of the $I_{\lambda}$-module $V_{\lambda}$ are of dimension 1 , again a contradiction.

Next we show that in general $\left(\mathcal{W}_{1}\right)$ implies $\left(\mathcal{W}_{2}^{\varepsilon}\right)$ for some $\varepsilon= \pm$.

Lemma 5.8. (i) Let $g \in S p(M)=S p_{2 n}(q)$. Then there is a g-invariant quadratic form on $M$ (that is, polarized to the symplectic form $(\cdot, \cdot)$ on $M)$.

(ii) Let $\kappa: O_{2 n}^{\epsilon}(q) \rightarrow\{ \pm 1\}$ be defined by $\kappa(g)=(-1)^{\operatorname{dim}_{\mathbb{F}_{q}} \operatorname{Ker}(g-1)}$. Then $\kappa$ is actually a group homomorphism, and $\operatorname{Ker}(\kappa)=\Omega_{2 n}^{\epsilon}(q)$.

Proof. (i) Let $g=s u$, where $s$ is the semisimple part and $u$ is the unipotent part of $g$. By [SS, Lemma 4.1], there is a $u$-invariant quadratic form $Q$ on $M$ that is 
polarized to $(\cdot, \cdot)$. Let $N=|s|$, and let $\tilde{Q}(x)=\sum_{i=0}^{N-1} Q\left(s^{i}(x)\right)$ for any $x \in M$. Then $\tilde{Q}$ is $g$-invariant and polarized to $(\cdot, \cdot)$ (since $N$ is odd).

(ii) Cf. Atlas, p. xii].

The map $\kappa$ defined in Lemma 5.8 ii) is called the quasideterminant.

Lemma 5.9. Let $G=S p_{2 n}(q)$ with $n \geq 2$, and consider a subgroup $H=O_{2 n}^{\alpha}(q)$ of $G$ for some $\alpha= \pm$. Let $\mu$ be a linear character of $H$. If $(n, q, \alpha)=(2,2,+)$, then assume in addition that $\mu=1_{H}$. Then $\mu$ is either $1_{H}$ or $\kappa$. If $\mu=1_{H}$, then all $G$-composition factors of $\operatorname{Ind}_{H}^{G}(\mu)$ are linear-Weil modules and satisfy $\mathcal{O}_{2}^{+}$. If $\mu=\kappa$, then all $G$-composition factors of $\operatorname{Ind}_{H}^{G}(\mu)$ are unitary-Weil modules and satisfy $\mathcal{O}_{2}^{-}$.

Proof. We fix some notation: $H_{\alpha}=O_{2 n}^{\alpha}(q), K_{\alpha}=\Omega_{2 n}^{\alpha}(q)$.

1) First we consider the case $\mu=1_{H}$ and prove the following formula:

$$
\operatorname{Ind}_{H_{+}}^{G}\left(1_{H_{+}}\right)+\operatorname{Ind}_{H_{-}}^{G}\left(1_{H_{-}}\right)=\tau_{n} .
$$

For, the left-hand side of (10) is exactly the permutation character of $G$ on the set $\Phi$ of quadratic forms on $M$ that are polarized to $(\cdot, \cdot)$. Let $g \in G$ be any element. By Lemma $5.8(\mathrm{i}), g$ fixes a point $Q \in \Phi$. Now $g$ fixes $Q^{\prime} \in \Phi$ if and only if $g$ fixes $\sqrt{Q+Q^{\prime}} \in \operatorname{Hom}_{\mathbb{F}_{q}}\left(M, \mathbb{F}_{q}\right)=M^{*}$. So the value of the left-hand side at $g$ is the number of $g$-fixed points on $M^{*}$, and therefore it equals $\tau_{n}(g)$. By Corollary 4.2 $\tau_{n}$ satisfies $\left(\mathcal{W}_{2}^{+}\right)$, so we are done with the case $\mu=1_{H}$.

2) Now we assume that $\mu_{H} \neq 1_{H}$. By assumption, in this case we have $(n, q, \alpha) \neq$ $(2,2,+)$. It follows that $K_{\alpha}$ is perfect, so $\mu=\kappa$. By Corollary 4.2 it suffices to prove

$$
\operatorname{Ind}_{H_{+}}^{G}\left(\left.\kappa\right|_{H_{+}}\right)+\operatorname{Ind}_{H_{-}}^{G}\left(\left.\kappa\right|_{H_{-}}\right)=\zeta_{n} .
$$

In view of (10), (11) is equivalent to the statement that the virtual character

$$
\phi:=\tau_{n}+\zeta_{n}-\left(\operatorname{Ind}_{K_{+}}^{G}\left(1_{K_{+}}\right)+\operatorname{Ind}_{K_{-}}^{G}\left(1_{K_{-}}\right)\right)
$$

is 0 .

First we show that $\phi(g) \geq 0$ for all $g \in G$. Indeed, assume $d_{g}:=\operatorname{dim}_{\mathbb{F}_{q}} \operatorname{Ker}(g-1)$ is even. Then

$$
\tau_{n}(g)+\zeta_{n}(g)=2 q^{d_{g}}=2 \tau_{n}(g)=\left(\operatorname{Ind}_{H_{+}}^{G}\left(2 \cdot 1_{H_{+}}\right)+\operatorname{Ind}_{H_{-}}^{G}\left(2 \cdot 1_{H_{-}}\right)\right)(g)
$$

(because of (10))

$$
\geq\left(\operatorname{Ind}_{K_{+}}^{G}\left(1_{K_{+}}\right)+\operatorname{Ind}_{K_{-}}^{G}\left(1_{K_{-}}\right)\right)(g)
$$

(as $\left(H_{\alpha}: K_{\alpha}\right)=2$ ), whence $\phi(g) \geq 0$. Assume $d_{g}$ is odd. Then $g$ cannot be contained in any $G$-conjugate of any $K_{ \pm}$by Lemma 5.8 (ii), so

$$
\left(\operatorname{Ind}_{K_{+}}^{G}\left(1_{K_{+}}\right)+\operatorname{Ind}_{K_{-}}^{G}\left(1_{K_{-}}\right)\right) \mid(g)=0 .
$$

Also, $\tau_{n}(g)+\zeta_{n}(g)=q^{d_{g}}+(-q)^{d_{g}}=0$, whence $\phi(g)=0$.

Since $\tau_{n}$, resp. $\zeta_{n}$, contains $1_{G}$ with multiplicity 2 , resp. $0,\left(\phi, 1_{G}\right)_{G}=0$. The latter formula, together with the above statement that $\phi(g) \geq 0$ for all $g \in G$, implies that $\phi \equiv 0$. 
Lemma 5.10. Let $G=S p_{2 n}(q)$ with $n \geq 2,(n, q) \neq(2,2),(3,2)$. Let $\mu$ be a linear character of $P_{1}^{\prime}$. Then $\mu=1_{P_{1}^{\prime}}$, and all $G$-composition factors of $\operatorname{Ind}_{P_{1}^{\prime}}^{G}(\mu)$ are linear-Weil modules and satisfy $\mathcal{O}_{2}^{+}$.

Proof. Since $n \geq 2$ and $(n, q) \neq(2,2)$, all $P_{1}^{\prime}$-orbits on $\operatorname{IBr}_{\ell}\left(Q_{1}\right)$ are of length $>1$. Hence $\operatorname{Ker}(\mu) \geq Q_{1}$ by Clifford's Theorem. Next, our assumption on $n, q$ implies that $P_{1}^{\prime} / Q_{1}=S p_{2 n-2}(q)$ is perfect. Thus $\mu=1_{P_{1}^{\prime}}$, whence $\operatorname{Ind}_{P_{1}^{\prime}}^{G}(\mu)=\tau_{n}-1_{G}$, and so we are done by Corollary 4.2 .

Recall that $I_{\lambda}=\operatorname{Stab}_{L_{1}}(\lambda)$ and $J_{\lambda}=S t a b_{L_{1}^{\prime}}(\lambda)$ for any $\lambda \in \operatorname{IBr}_{\ell}\left(Q_{1}\right)$.

Theorem 5.11. Let $G=S p_{2 n}(q)$ with $n \geq 3,(n, q) \neq(3,2),(4,2)$, and $V$ a nontrivial irreducible $\mathbb{F} G$-module. Suppose that $V$ satisfies $\left(\mathcal{W}_{1}\right)$. Then exactly one of the following holds:

(i) If $\lambda$ is any nontrivial linear $Q_{1}$-character occurring in $\left.V\right|_{Q_{1}}$, then $\lambda \in \mathcal{O}_{2}^{+} \cup$ $\mathcal{O}_{2}^{-}$, and $I_{\lambda}=O_{2 n-2}^{ \pm}(q)$ acts via the character $\kappa$ on the homogeneous component $V_{\lambda}$. Furthermore, $V$ satisfies $\left(\mathcal{W}_{2}^{-}\right)$.

(ii) If $\lambda$ is any nontrivial linear $Q_{1}$-character occurring in $\left.V\right|_{Q_{1}}$, then all composition factors of the $J_{\lambda}$-module $V_{\lambda}$ are trivial. Furthermore, $V$ satisfies $\left(\mathcal{W}_{2}^{+}\right)$.

In particular, $\left(\mathcal{W}_{1}\right)$ implies $\left(\mathcal{W}_{2}^{\varepsilon}\right)$ for some $\varepsilon= \pm$.

Proof. 1) Define $V_{1}=\sum_{\lambda \in \mathcal{O}_{1}} V_{\lambda}, V_{2}^{\varepsilon}=\sum_{\lambda \in \mathcal{O}_{2}^{\varepsilon}} V_{\lambda}$, where $V_{\lambda}$ is the $\lambda$-homogeneous component of $\left.V\right|_{Q_{1}}$ for any $\lambda \in \operatorname{IBr}_{\ell}\left(Q_{1}\right)$. Since $L_{1}$ is transitive on each of these orbits $\mathcal{O}_{1}, \mathcal{O}_{2}^{\varepsilon}$, the $L_{1}$-module $V_{1}$, resp. $V_{2}^{\varepsilon}$, is $\operatorname{Ind}_{I_{\lambda}}^{L_{1}}\left(V_{\lambda}\right)$. Observe that $L_{1}=$ $L_{1}^{\prime} \times \mathbb{Z}_{q-1}$. Moreover, $L_{1}^{\prime}$ acts transitively on $\mathcal{O}_{1}$, meanwhile each $\mathcal{O}_{2}^{\varepsilon}$ splits into $q-1 L_{1}^{\prime}$-orbits. It follows by Mackey's formula that $V_{1}=\operatorname{Ind}_{J_{\lambda}}^{L_{1}^{\prime}}\left(V_{\lambda}\right)$ and $V_{2}^{\varepsilon}=$ $(q-1) \operatorname{Ind}_{J_{\lambda}}^{L_{1}^{\prime}}\left(V_{\lambda}\right)$ as $L_{1}^{\prime}$-modules.

By assumption, all $J_{\lambda}$-composition factors on $V_{\lambda}$ are of dimension 1. Consider any $\lambda \in \mathcal{O}_{2}^{\varepsilon}$. Then Lemma 5.9 implies that the Brauer character of $J_{\lambda}=O_{2 n-2}^{\varepsilon}(q)$ on $V_{\lambda}$ is $a_{\varepsilon} \cdot 1_{J_{\lambda}}+b_{\varepsilon} \cdot \kappa$ for some $a_{\varepsilon}, b_{\varepsilon} \in \mathbb{Z}$, and all $L_{1}^{\prime}$-composition factors of $V_{2}^{\varepsilon}$ are (linear or unitary) Weil modules. Next let $\lambda \in \mathcal{O}_{1}$. Then Lemma 5.10 implies that the Brauer character of $J_{\lambda}$ on $V_{\lambda}$ is $c \cdot 1_{J_{\lambda}}$ for some $c \in \mathbb{Z}$, and all $L_{1}^{\prime}$-composition factors of $V_{1}$ are linear-Weil modules.

Observe that $V=C_{V}\left(Q_{1}\right) \oplus V_{1} \oplus V_{2}^{+} \oplus V_{2}^{-}$as a $P_{1}$-module. By Lemma 2.1 all $L_{1}^{\prime}$-composition factors of $C_{V}\left(Q_{1}\right)$ are also Weil modules.

2) Let $\mathfrak{W}_{+}$, resp. $\mathfrak{W}_{-}$, be the set of all linear-Weil, resp. unitary-Weil, irreducible Brauer characters of $L_{1}^{\prime}$ together with the trivial character. Observe that if $\rho \in$ $\mathfrak{W}_{+} \cap \mathfrak{W}_{-}$, then $\rho$ is trivial. Indeed, $\rho$ satisfies both $\left(\mathcal{W}_{2}^{+}\right)$and $\left(\mathcal{W}_{2}^{-}\right)$by Corollary 4.2. Hence, if $Z_{2}$ is a standard subgroup of type $Z_{2}$ for $L_{1}^{\prime}$, then all $Z_{2}$-characters in $\left.\rho\right|_{Z_{2}}$ are contained in the orbit $\mathcal{O}_{1}$. It follows that $\operatorname{Ker}(\rho)$ contains the subgroup $\left\{[0, C] \in Z_{2} \mid C \in \mathcal{H}_{2}^{0}(q)\right\}$ of order $q$, and so $\rho$ is trivial by irreducibility. Notice that we have used the assumption $n \geq 3$ here.

Given any $\mathbb{F} L_{1}^{\prime}$-module $M$ and any family $\mathcal{X}$ of simple $L_{1}^{\prime}$-modules, there is a largest submodule $M(\mathcal{X})$ of $M$ with all composition factors belonging to $\mathcal{X}$; cf. GMST, Lemma 4.3].

3) Let $\lambda \in \mathcal{O}_{2}^{\varepsilon}$. Then $I_{\lambda}=J_{\lambda}=O_{2 n-2}^{\varepsilon}(q)$, and the perfect subgroup $\Omega_{2 n-2}^{\varepsilon}(q)$ of $J_{\lambda}$ acts trivially on $V_{\lambda}$. Also, $Q_{1}$ acts scalarly on $V_{\lambda}$ (via the character $\lambda$ ). Hence, the $K_{\lambda}$-module $V_{\lambda}$ is semisimple, where $K_{\lambda}:=\operatorname{Stab}_{P_{1}}(\lambda)$. In particular, $V_{\lambda}=V_{\lambda}^{+} \oplus V_{\lambda}^{-}$, where $V_{\lambda}^{+}$, resp. $V_{\lambda}^{-}$, affords the $J_{\lambda}$-character $a_{\varepsilon} \cdot 1_{J_{\lambda}}$, resp. $b_{\varepsilon} \cdot \kappa$. 
Let $T=\left[V, Q_{1}\right], T_{+}=V_{1} \oplus \operatorname{Ind}_{K_{\lambda_{1}}}^{P_{1}}\left(V_{\lambda_{1}}^{+}\right) \oplus \operatorname{Ind}_{K_{\lambda_{2}}}^{P_{1}}\left(V_{\lambda_{2}}^{+}\right), T_{-}=\operatorname{Ind}_{K_{\lambda_{1}}}^{P_{1}}\left(V_{\lambda_{1}}^{-}\right) \oplus$ $\operatorname{Ind}_{K_{\lambda_{2}}}^{P_{1}}\left(V_{\lambda_{2}}^{-}\right)$, for fixed $\lambda_{1} \in \mathcal{O}_{2}^{+}$and $\lambda_{2} \in \mathcal{O}_{2}^{-}$. Then $T=T_{+} \oplus T_{-}$as $P_{1}$-modules.

Claim that $T\left(\mathfrak{W}_{+}\right)=T_{+}$. Indeed, $T\left(\mathfrak{W}_{+}\right) \supseteq T_{+}$by Lemmas 5.9 and 5.10 Assume that $T\left(\mathfrak{W}_{+}\right) \neq T_{+}$. Then $T_{-} \simeq T / T_{+}$contains a simple $L_{1}^{\prime}$-submodule with character $\rho \in \mathfrak{W}_{+}$. On the other hand, the construction of $T_{-}$implies by Lemma 5.9 that $\rho \in \mathfrak{W}_{-}$. Hence $\rho=1_{L_{1}^{\prime}}$ according to 2$)$. Thus $T_{-}$contains the trivial $L_{1}^{\prime}$-module as a submodule. We may therefore assume that

$$
0 \neq \operatorname{Hom}_{L_{1}^{\prime}}\left(1_{L_{1}^{\prime}}, \operatorname{Ind}_{J_{\lambda_{i}}}^{L_{1}^{\prime}}(\kappa)\right) \simeq \operatorname{Hom}_{J_{\lambda_{i}}}\left(1_{J_{\lambda_{i}}}, \kappa\right)
$$

for some $i=1$ or 2 . But the last hom-space is 0 as $J_{\lambda_{i}}=O_{2 n-2}^{ \pm}(q)$ and $\kappa=-1$ on $O_{2 n-2}^{ \pm}(q) \backslash \Omega_{2 n-2}^{ \pm}(q)$, a contradiction.

Thus $T\left(\mathfrak{W}_{+}\right)=T_{+}$; in particular, $T\left(\mathfrak{W}_{+}\right)$is $P_{1}$-stable.

4) Let $U=C_{V}\left(Q_{1}\right)$. Since $Q_{1}$ acts trivially on $U$ and $L_{1}=L_{1}^{\prime} \times \mathbb{Z}_{q-1}, U\left(\mathfrak{W}_{+}\right)$ is also $P_{1}$-stable. It follows that $V\left(\mathfrak{W}_{+}\right)=T\left(\mathfrak{W}_{+}\right) \oplus U\left(\mathfrak{W}_{+}\right)$is $P_{1}$-stable. On the other hand, it is clear that $V\left(\mathfrak{W}_{+}\right)$is stable under a standard subgroup $S:=S p_{2}(q)$ that centralizes $L_{1}^{\prime}$. Consequently, $V\left(\mathfrak{W}_{+}\right)$is stable under $\left\langle P_{1}, S\right\rangle=G$, and so $V\left(\mathfrak{W}_{+}\right)=0$ or $V$ by irreducibility.

Assume $V\left(\mathfrak{W}_{+}\right)=0$. Then $V_{1}=0$ and $a_{\varepsilon}=0$ for all $\varepsilon= \pm$. Also, $T=T_{-}$, and so all $L_{1}^{\prime}$-composition factors of $T$ are unitary-Weil modules. The same holds for $U$ as well by Lemma 2.1. Hence the $L_{1}^{\prime}$-module $V$ satisfies $\left(\mathcal{W}_{2}^{-}\right)$by Corollary 4.2 and (i) holds.

Assume $V\left(\mathfrak{W}_{+}\right)=V$. Then $T_{-}=0$, and $b_{\varepsilon}=0$ for all $\varepsilon= \pm$. Also, all $L_{1}^{\prime}$ composition factors of $V$ are linear-Weil modules. Hence the $L_{1}^{\prime}$-module $V$ satisfies $\left(\mathcal{W}_{2}^{+}\right)$by Corollary 4.2 and (ii) holds.

Finally, the argument in 2) (applied to $G$ in place of $L_{1}^{\prime}$ ) shows that exactly one of the cases (i), (ii) holds for $V$, since $V$ is nontrivial.

Let $b^{\epsilon}(n, q)$ denote the improved Landazuri-Seitz-Zalesskii lower bound for faithful $\mathbb{F} \Omega_{2 n}^{\epsilon}(q)$-representations as stated in [Ho]. In particular,

$$
b^{-}(n, q)=\frac{\left(q^{n}+1\right)\left(q^{n-1}-q\right)}{q^{2}-1}-1
$$

if $n \geq 4$, except for $(n, q)=(4,2),(4,4),(5,2)$, where one has to decrease the bound by 2. Define

$$
\mathfrak{d}(n, q)= \begin{cases}b^{-}(n-1, q) \cdot q^{n-1}\left(q^{n-1}-1\right)(q-1) / 2, & \text { if } n \geq 5 \\ \left(q^{4}-1\right)\left(q^{3}-1\right) q^{2}, & \text { if } n=4\end{cases}
$$

Observe that

$$
\mathfrak{d}(n, q)=\frac{q^{4 n-6}}{2} \cdot\left(1-\frac{1}{q}+O\left(\frac{1}{q^{2}}\right)\right)
$$

provided that $n \geq 5$.

Theorem 5.12. Let $G=S p_{2 n}(q)$ with $n \geq 4$ and $(n, q) \neq(4,2)$, $(5,2)$, and let $V \in \operatorname{IBr}_{\ell}(G)$. Then either $\operatorname{dim}(V) \geq \mathfrak{d}(n, q)$, or $V$ satisfies $\left(\mathcal{W}_{1}\right)$.

Proof. Assume the contrary: $\operatorname{dim}(V)<\mathfrak{d}(n, q)$, but $V$ violates $\left(\mathcal{W}_{1}\right)$. Then there is a nontrivial linear character $\lambda$ of $Q_{1}$ such that $I_{\lambda}$ has a composition factor of dimension $>1$ on the homogeneous component $V_{\lambda}$ of $V$. 
First assume that $\lambda \in \mathcal{O}_{2}^{\varepsilon}$. Then $I_{\lambda}=\Omega_{2 n-2}^{\varepsilon}(q): 2$ and $\Omega_{2 n-2}^{\varepsilon}(q)$ is simple since $n \geq 4$. The assumption on $\lambda$ implies that $\operatorname{dim}(V) \geq\left|\mathcal{O}_{2}^{\varepsilon}\right| \cdot b^{\varepsilon}(n-1, q) \geq \mathfrak{d}(n, q)$, a contradiction. Hence $\lambda \in \mathcal{O}_{1}$.

Define $t=4$ for $n \geq 5$ and $t=3$ for $n=4$. Consider the standard subgroup

$$
H=\operatorname{Stab}_{G}\left(e_{t+1}, \ldots, e_{n}, f_{t+1}, \ldots, f_{n}\right) \simeq S p_{2 t}(q)
$$

of $G$ and the subgroup $Z_{t}=\operatorname{Stab}_{H}\left(e_{1}, \ldots, e_{t}\right)$ of $H$. Let $K$ denote the kernel of $K_{\lambda}:=\operatorname{Stab}_{P_{1}}(\lambda)$ on $V_{\lambda}$. We will assume that $P_{1}^{\prime}=S \operatorname{Stab} b_{G}\left(e_{t+1}\right)$. Then we may identify $K_{\lambda} \cap P_{1}^{\prime}$ with $\operatorname{Stab}_{G}\left(e_{t+1}, f_{t}+\left\langle e_{t+1}\right\rangle_{\mathbb{F}_{q}}\right)$. In this case $K_{\lambda} \cap Z_{t}=S t a b_{Z_{t}}\left(f_{t}\right)$ is the subgroup $A$ defined in Lemma 5.5 when $n \geq 5$ and in Lemma 5.6 when $n=4$. Assume the subgroup $A_{0}$ of $A$ (as defined in Lemma 5.5, respectively in Lemma 5.6) does not act trivially on $V_{\lambda}$. By Lemma 5.3. $\left.V\right|_{Z_{t}}$ contains $\operatorname{Ind}_{A}^{Z_{t}}\left(V_{\lambda}\right)$ (as $Z_{t}<P_{1}^{\prime}$ ), and $\operatorname{Ind}_{A}^{Z_{t}}\left(V_{\lambda}\right)$ intersects some orbit $\mathcal{O}_{t}^{\epsilon}$ of $Z_{t}$-characters, according to Lemma 5.5, respectively Lemma 5.6. In other words, the $\mathbb{F} P_{t}$-module $V$ does not satisfy $\left(\mathcal{W}_{t-1}\right)$. By Lemma $3.4(i i),\left.V\right|_{Z_{n}}$ does not satisfy $\left(\mathcal{W}_{t-1}\right)$. In other words, $\left.V\right|_{Z_{n}}$ affords some orbit $\mathcal{O}_{s}^{\epsilon}$ of $Z_{n}$-characters, with $s \geq t$. Observe that

$$
\left|\mathcal{O}_{1}^{ \pm}\right| \leq\left|\mathcal{O}_{2}^{ \pm}\right| \leq\left|\mathcal{O}_{3}^{ \pm}\right| \leq \ldots \leq\left|\mathcal{O}_{n}^{ \pm}\right|
$$

if $q \geq 4$ or if $n$ is odd, and

$$
\left|\mathcal{O}_{1}^{ \pm}\right| \leq\left|\mathcal{O}_{2}^{ \pm}\right| \leq \ldots \leq\left|\mathcal{O}_{n-2}^{ \pm}\right| \leq \min \left\{\left|\mathcal{O}_{n-1}^{ \pm}\right|,\left|\mathcal{O}_{n}^{ \pm}\right|\right\}
$$

if $q=2$ and $n$ is even. Since we are assuming $(n, q) \neq(4,2)$, it follows that $\operatorname{dim}(V) \geq\left|\mathcal{O}_{4}^{ \pm}\right|>\mathfrak{d}(n, q)$ if $n \geq 5$, and $\operatorname{dim}(V) \geq\left|\mathcal{O}_{3}\right|=\mathfrak{d}(n, q)$ if $n=4$, again a contradiction.

We have shown that $K \geq A_{0}$. Observe that $K_{\lambda} \cap P_{1}^{\prime}=Q_{1}: J_{\lambda}$, where $J_{\lambda}:=$ $S t a b_{L_{1}^{\prime}}(\lambda)=\operatorname{Stab}_{G}\left(e_{t+1}, f_{t+1}, f_{t}\right)$ plays the role of the subgroup $P_{1}^{\prime}$ in the standard subgroup $\operatorname{Stab}_{G}\left(e_{t+1}, f_{t+1}\right) \simeq S p_{2 n-2}(q)$. Next, $J_{\lambda}=\left[q^{2 n-3}\right]: S$, where $S=$ $\operatorname{Stab}_{G}\left(e_{t}, e_{t+1}, f_{t}, f_{t+1}\right) \simeq S p_{2 n-4}(q)$. Clearly, $A_{0}<K \cap S$. Thus $K \cap S$ is a normal subgroup of $S \simeq S p_{2 n-4}(q)$ of order $\geq\left|A_{0}\right| \geq q$. Since $n \geq 4$ and $(n, q) \neq(4,2)$, it follows that $K \geq S$. Now $K \cap J_{\lambda}$ is a normal subgroup of $J_{\lambda}=\left[q^{2 n-3}\right]: S p_{2 n-4}(q)$ that contains $S p_{2 n-4}(q)$. Since $n \geq 4$, this implies that $K \geq J_{\lambda}$. Observe that $Q_{1}$ acts on $V_{\lambda}$ as scalars \pm 1 , and $I_{\lambda} / J_{\lambda} \simeq \mathbb{Z}_{q-1}$. It follows that all composition factors of the $I_{\lambda}$-module $V_{\lambda}$ are of dimension 1 , contrary to the choice of $\lambda$.

Remark 5.13. Theorem 5.12 remains true for $(n, q)=(5,2)$ if we set $\mathfrak{d}(5,2)=$ $\left|\mathcal{O}_{2}^{+}\right| \cdot b^{+}(4,2)=3808$.

\section{LOW-DIMENSIONAL COMPLEX REPRESENTATIONS OF $S p_{2 n}(q)$}

Irreducible complex representations of $G=S p_{2 n}(q)$ have been classified up to degree $\left(q^{2 n}-1\right) / 2(q+1)$; cf. [TZ1, Theorem 5.5]. In this section we extend this classification up to degree $\left(q^{2 n}-1\right)\left(q^{n-1}-1\right)\left(q^{n-1}-q^{2}\right) / 2\left(q^{4}-1\right)$ (generically). Since the argument is pretty much the same as in [TZ1], we will only sketch the proof. Since $q$ is even, we may identify $G$ with its dual group. Lusztig's classification of irreducible characters of $G[\mathrm{~L}]$ parametrizes $\chi \in \operatorname{Irr}(G)$ by a pair $\left((s), \chi_{u}\right)$, where $(s)$ is a $G$-conjugacy class of a semisimple element $s \in G$ and $\chi_{u}$ is a unipotent character of $C_{G}(s)$. In this case $\chi(1)=\left(G: C_{G}(s)\right)_{2^{\prime}} \cdot \chi_{u}(1)$. Furthermore, unipotent characters of $G$ are parametrized by symbols $\left(\begin{array}{l}\lambda \\ \mu\end{array}\right)$ of a certain kind as explained in C] $§ 13.8]$. 
Theorem 6.1. Let $G=S p_{2 n}(q), n \geq 1$, and let $\chi \in \operatorname{Irr}(G)$.

(A) Suppose $n \geq 3$ and $\chi$ is unipotent. Then one of the following holds:

(A1) $\chi \in\left\{1_{G}, \alpha_{n}, \beta_{n}, \rho_{n}^{1}, \rho_{n}^{2}\right\}$.

(A2) $n=3, q=2$, and $\chi(1)$ is at least $q^{4}(q-1)^{2}\left(q^{2}+q+1\right) / 2$, which is the degree of the unipotent character labelled by $\left(\begin{array}{ccc}0 & 1 & 2 \\ & 1\end{array}\right)$.

(A3) $n=3, q>2$, and $\chi(1)$ is at least $q^{2}\left(q^{4}+q^{2}+1\right)$, which is the degree of the unipotent character labelled by $\left(\begin{array}{c}0 \\ n-1\end{array}\right)$.

(A4) $n \geq 4$, and $\chi(1)$ is at least $\left(q^{2 n}-1\right)\left(q^{n-1}-1\right)\left(q^{n-1}-q^{2}\right) / 2\left(q^{4}-1\right)$, which is the degree of the unipotent character labelled by $\left(\begin{array}{ccc}0 & 2 & n-1 \\ -\end{array}\right)$.

(B) Suppose $\chi$ is not unipotent. Then $\chi(1) \geq\left(q^{2 n}-1\right) /(q+1)$. Moreover, if $n \geq 3$ and $(n, q) \neq(3,2)$, then one of the following holds:

(B1) $\chi(1) \geq\left(q^{2 n}-1\right)\left(q^{2 n-2}-1\right) /\left(q^{2}-1\right)(q+1)$.

(B2) $\chi \in\left\{\tau_{n}^{i} \mid 1 \leq i \leq q / 2-1\right\} \cup\left\{\zeta_{n}^{i} \mid 1 \leq i \leq q / 2\right\}$.

(B3) $n=3,4, \chi(1)=\prod_{i=1}^{n}\left(q^{i}+\gamma^{i}\right), \gamma= \pm$, and $\chi$ is parametrized by $\left((s), 1_{C}\right)$, where $C:=C_{G}(s) \simeq G L_{n}^{\gamma}(q)$.

(B4) $n=5, \chi(1)=\prod_{i=1}^{5}\left(q^{i}+(-1)^{i}\right)$, and $\chi$ is parametrized by $\left((s), 1_{C}\right)$, where $C:=C_{G}(s) \simeq G U_{5}(q)$.

(B5) $n \geq 4, q=2, \chi(1)=\left(q^{2 n}-1\right)\left(q^{n-1}-\gamma\right)\left(q^{n-1}-\gamma q\right) / 2\left(q^{2}-1\right)(q+1)$, $\gamma= \pm$, and $\chi$ is parametrized by $\left((s), \chi_{u}\right)$, where $C_{G}(s) \simeq \mathbb{Z}_{q+1} \times S p_{2 n-2}(q)$ and $\chi_{u}=1 \otimes \alpha_{n-1}$, resp. $1 \otimes \beta_{n-1}$, for $\gamma=+$, resp. for $\gamma=-$.

Proof. (A) Assume that $\chi$ is unipotent. Define

$$
D(n)=\left(q^{2 n}-1\right)\left(q^{n-1}-1\right)\left(q^{n-1}-q^{2}\right) / 2\left(q^{4}-1\right)
$$

when $n \geq 4$ and $D(3)=q^{2}\left(q^{4}+q^{2}+1\right)$. Notice that $D(n) / D(n-1)<q^{5}$ and $D(n)<q^{4 n-5} / 2$, provided that $n \geq 4$. Also, $D(n)>q^{4 n-6} / 2$ if $n \geq 8$ and $D(n)>q^{4 n-7} / 2$ if $n \geq 5$.

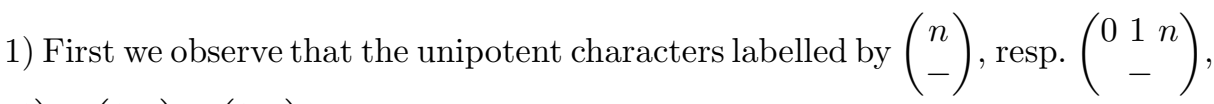

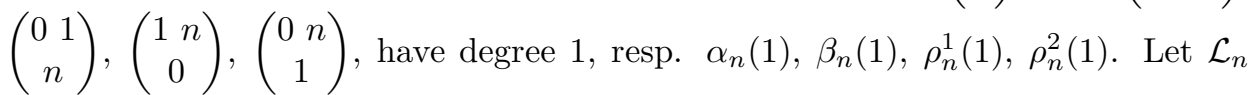
be the set of these five symbols. We will actually prove by induction on $n \geq 3$ and $(n, q) \neq(3,2)$ that if $\chi=\chi^{\lambda, \mu}$ with $\left(\begin{array}{l}\lambda \\ \mu\end{array}\right) \notin \mathcal{L}_{n}$, then $\chi(1) \geq D(n)$. The induction base $n=3$ can be checked using $\mathrm{Lu}$, and the case $(n, q)=(4,2)$ can be checked using Atlas. So for the induction step we will assume that $n \geq 4$ and that $(n, q) \neq(4,2)$. Assume that $\chi$ is the unipotent character $\chi^{\lambda, \mu}$ labelled by $\left(\begin{array}{l}\lambda \\ \mu\end{array}\right)=$ $\left(\begin{array}{ccccc}\lambda_{1} & \lambda_{2} & \lambda_{3} & \ldots & \lambda_{a} \\ \mu_{1} & \mu_{2} & \ldots & \mu_{b}\end{array}\right)$, where $0 \leq \lambda_{1}<\lambda_{2}<\ldots<\lambda_{a}, 0 \leq \mu_{1}<\mu_{2}<\ldots<\mu_{b}, a-b$ is odd and positive, $\left(\lambda_{1}, \mu_{1}\right) \neq(0,0)$, and

$$
\sum_{i} \lambda_{i}+\sum_{j} \mu_{j}-\left[\left(\frac{a+b-1}{2}\right)^{2}\right]=n .
$$


The integer $n$ is called the rank of $\left(\begin{array}{l}\lambda \\ \mu\end{array}\right)$. We will assume $\left(\begin{array}{l}\lambda \\ \mu\end{array}\right) \notin \mathcal{L}_{n}$ and proceed by induction on $b$.

2) Here we consider the case $b=0$. Then $a \geq 1$ is odd. We may assume that $a \geq 3$, and $\lambda \neq(0,1, n),(0,2, n-1)$.

First we assume that $a=3$. If $\lambda_{1}=0$, then $\lambda=(0, k, n+1-k)$, where $3 \leq k<(n+1) / 2$; in particular $n \geq 6$. It is easy to check that $\chi(1)>D(n)$ in this case. If $\lambda_{1} \geq 1$, then $\chi(1)>q^{4 n-4} / 2>D(n)$, cf. [TZ1, p. 2119]. Now we may assume that $a \geq 5$.

Suppose $\lambda_{1} \geq 1$. Consider the unipotent character $\chi^{\prime}$ labelled by the symbol $\left(\begin{array}{c}\lambda^{\prime} \\ \mu^{\prime}\end{array}\right)$ of rank $n-1$, where $\lambda^{\prime}=\left(\lambda_{1}-1, \lambda_{2}, \ldots, \lambda_{a}\right)$, and $\mu^{\prime}=\mu$. Observe that $\chi(1) / \chi^{\prime}(1) \geq q^{2\left(n-\lambda_{1}\right)} / 2$. But $n-\lambda_{1} \geq(a-1) \lambda_{1}+\left(a^{2}-1\right) / 4 \geq 10$, and $\chi^{\prime}(1) \geq$ $D(n-1)$ by induction hypothesis. It follows that $\chi(1)>q^{19} D(n-1)>D(n)$. Now we may assume that $\lambda_{1}=0$.

Next we assume that $\lambda_{i}-\lambda_{i-1} \geq 2$ for some $i \geq 2$. Consider the unipotent character $\chi^{\prime}$ labelled by the symbol $\left(\begin{array}{c}\lambda^{\prime} \\ \mu^{\prime}\end{array}\right)$ of rank $n-1$, where $\lambda^{\prime}=\left(\lambda_{1}, \ldots, \lambda_{i-1}, \lambda_{i}-\right.$ $\left.1, \lambda_{i+1}, \ldots, \lambda_{a}\right)$, and $\mu^{\prime}=\mu$. Observe that $\chi(1) / \chi^{\prime}(1)>q^{2\left(n-\lambda_{i}\right)} / 2$, and $n-1 \geq 6$. By induction hypothesis, $\chi^{\prime}(1) \geq D(n-1)$. Hence, if $n-\lambda_{i} \geq 3$, then $\chi(1)>$ $q^{5} D(n-1)>D(n)$. If $n-\lambda_{i} \leq 2$, then actually $i=a=5, \lambda=(0,1,2,3, n-2)$ and so $\chi(1)>D(n)$.

Now we may assume that $\lambda=(0,1,2, \ldots, a-2, a-1)$. Consider $\chi^{\prime}=\chi^{\lambda^{\prime}, \mu^{\prime}}$, where $\lambda^{\prime}=(0,1,2, \ldots, a-4, a-3)$, and $\mu^{\prime}=\mu$. Then $\chi(1) / \chi^{\prime}(1) \geq q^{4 n-2}$, and so $\chi(1)>q^{4 n-2}>D(n)$.

3) From now on we may assume that $b \geq 1$. At this point we suppose that $\left(\lambda_{1}, \mu_{1}\right) \neq(1,0)$ and $\lambda_{1} \geq 1$. Consider the character $\chi^{\prime}$ labelled by the symbol $\left(\begin{array}{c}\lambda^{\prime} \\ \mu^{\prime}\end{array}\right)$ of rank $n-1$, where $\lambda^{\prime}=\left(\lambda_{1}-1, \lambda_{2}, \ldots, \lambda_{a}\right)$ and $\mu^{\prime}=\mu$. If $n-\lambda_{1} \leq 2$,

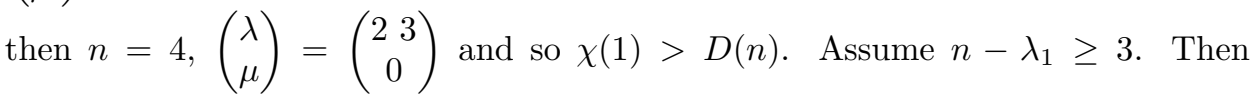
$\chi(1) / \chi^{\prime}(1)>q^{2\left(n-\lambda_{1}\right)} / 2 \geq q^{5}$. In the case $\left(\begin{array}{c}\lambda^{\prime} \\ \mu^{\prime}\end{array}\right) \in \mathcal{L}_{n-1}$ one can easily check that $\chi(1)>D(n)$. If $\left(\begin{array}{l}\lambda^{\prime} \\ \mu^{\prime}\end{array}\right) \notin \mathcal{L}_{n-1}$, then $\chi^{\prime}(1)>D(n-1)$ by induction hypothesis, whence $\chi(1)>D(n)$.

Similarly, for $\left(\lambda_{1}, \mu_{1}\right) \neq(0,1)$ and $\mu_{1} \geq 1$, we consider the character $\chi^{\prime}$ labelled by the symbol $\left(\begin{array}{c}\lambda^{\prime} \\ \mu^{\prime}\end{array}\right)$ of rank $n-1$, where $\lambda^{\prime}=\lambda$ and $\mu^{\prime}=\left(\mu_{1}-1, \mu_{2}, \ldots, \mu_{b}\right)$. If $n-\mu_{1} \leq 2$, then one directly checks that $\chi(1)>D(n)$. Assume $n-\mu_{1} \geq 3$. Then $\chi(1) / \chi^{\prime}(1)>q^{2\left(n-\mu_{1}\right)} / 2 \geq q^{5}$. In the case $\left(\begin{array}{c}\lambda^{\prime} \\ \mu^{\prime}\end{array}\right) \in \mathcal{L}_{n-1}, \chi(1)>D(n)$ also holds. If $\left(\begin{array}{c}\lambda^{\prime} \\ \mu^{\prime}\end{array}\right) \notin \mathcal{L}_{n-1}$, then $\chi^{\prime}(1)>D(n-1)$ by induction hypothesis, whence $\chi(1)>D(n)$.

It remains to consider the case where $\left\{\lambda_{1}, \mu_{1}\right\}=\{0,1\}$. 
4) Here we suppose that $\left(\lambda_{1}, \mu_{1}\right)=(1,0)$ but $\lambda \neq(1,2, \ldots, a)$. Then there exists an $i \geq 2$ such that $\lambda_{i} \geq \lambda_{i-1}+2$. If $a=2$, then $\left(\begin{array}{l}\lambda \\ \mu\end{array}\right) \in \mathcal{L}_{n}$, hence $a \geq 3$. Consider the character $\chi^{\prime}$ labelled by the symbol $\left(\begin{array}{l}\lambda^{\prime} \\ \mu^{\prime}\end{array}\right)$ of rank $n-1$, where $\lambda^{\prime}=\left(\lambda_{1}, \ldots, \lambda_{i-1}, \lambda_{i}-1, \lambda_{i+1}, \ldots, \lambda_{a}\right)$, and $\mu^{\prime}=\mu$. Since $a \geq 3,\left(\begin{array}{c}\lambda^{\prime} \\ \mu^{\prime}\end{array}\right) \notin \mathcal{L}_{n-1}$ and so $\chi^{\prime}(1)>D(n-1)$. Observe that $\chi(1) / \chi^{\prime}(1)>q^{2\left(n-\lambda_{i}+1\right)} / 2$; cf. [TZ1, p. 2121]. Therefore, if $n-\lambda_{i} \geq 2$, then $\chi(1) / \chi^{\prime}(1)>q^{5}$ and we are done. Assume $n-\lambda_{i} \leq 1$. Then $i=a \geq 3$. If $n-\lambda_{a}=1$, then for $n \geq 5$,

$$
\begin{gathered}
\frac{\chi(1)}{\chi^{\prime}(1)}=\frac{q^{2 n}-1}{q^{2 \lambda_{a}}-1} \cdot \prod_{i^{\prime}<a} \frac{q^{\lambda_{a}}-q^{\lambda_{i^{\prime}}}}{q^{\lambda_{a}-1}-q^{\lambda_{i^{\prime}}}} \cdot \prod_{j} \frac{q^{\lambda_{a}}+q^{\mu_{j}}}{q^{\lambda_{a}-1}+q^{\mu_{j}}} \\
\geq \frac{q^{2 n}-1}{q^{2 n-2}-1} \cdot \frac{q^{n-1}-q}{q^{n-2}-q} \cdot \frac{q^{n-1}-q^{2}}{q^{n-2}-q^{2}} \cdot \frac{q^{n-1}+1}{q^{n-2}+1}
\end{gathered}
$$

and $\chi^{\prime}(1)>D(n-1)$, whence $\chi(1)>D(n)$. Assume that $n \leq \lambda_{a}$. Then $\left(\begin{array}{l}\lambda \\ \mu\end{array}\right)=$

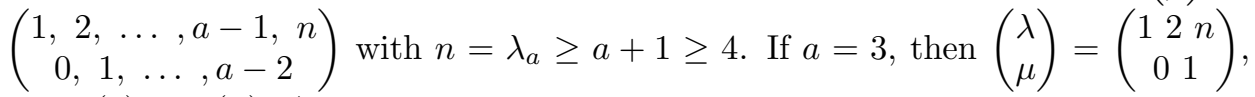
and $\chi(1)>D(n)$. Assume $a \geq 4$. In this case

$$
\begin{aligned}
\chi(1) / \chi^{\prime}(1) & =\prod_{j=1}^{a-1}\left(\frac{q^{n}-q^{j}}{q^{n-1}-q^{j}} \cdot \frac{q^{n}+q^{j-1}}{q^{n-1}+q^{j-1}}\right) \\
& =\frac{\left(q^{n}+1\right)\left(q^{n-1}-1\right)}{\left(q^{n-a}-1\right)\left(q^{n-a+1}+1\right)}>q^{2 a-2} \geq q^{6},
\end{aligned}
$$

and we are again done.

Similarly, suppose that $\left(\lambda_{1}, \mu_{1}\right)=(0,1)$ but $\mu \neq(1,2, \ldots, b)$; in particular, $b \geq 2$. Then there exists an index $j \geq 2$ such that $\mu_{j} \geq \mu_{j-1}+2$. Consider the character $\chi^{\prime}$ labelled by the symbol $\left(\begin{array}{l}\lambda^{\prime} \\ \mu^{\prime}\end{array}\right)$ of rank $n-1$, where $\lambda^{\prime}=\lambda$ and $\mu^{\prime}=\left(\mu_{1}, \ldots, \mu_{j-1}, \mu_{j}-1, \mu_{j+1}, \ldots, \mu_{b}\right)$. Since $b \geq 2,\left(\begin{array}{c}\lambda^{\prime} \\ \mu^{\prime}\end{array}\right) \notin \mathcal{L}_{n-1}$ and so $\chi^{\prime}(1)>$ $D(n-1)$. Observe that $\chi(1) / \chi^{\prime}(1)>q^{2\left(n-\mu_{j}+1\right)} / 2$; cf. [TZ1, p. 2122]. Therefore, if $n-\mu_{j} \geq 2$, then $\chi(1) / \chi^{\prime}(1)>q^{5}$ and we are done. Assume $n-\mu_{j} \leq 1$. Then $j=b$. If $n-\mu_{b}=1$, then $\left(\lambda_{1}, \lambda_{2}\right)=(0,1)$,

$$
\begin{gathered}
\frac{\chi(1)}{\chi^{\prime}(1)}=\frac{q^{2 n}-1}{q^{2 \mu_{b}}-1} \cdot \prod_{i} \frac{q^{\lambda_{i}}+q^{\mu_{b}}}{q^{\lambda_{i}}+q^{\mu_{b}-1}} \cdot \prod_{j^{\prime}<b} \frac{q^{\mu_{b}}-q^{\mu_{j^{\prime}}}}{q^{\mu_{b}-1}-q^{\mu_{j^{\prime}}}} \\
\quad \geq \frac{q^{2 n}-1}{q^{2 n-2}-1} \cdot \frac{q^{n-1}+1}{q^{n-2}+1} \cdot \frac{q^{n-1}+q}{q^{n-2}+q} \cdot \frac{q^{n-1}-q}{q^{n-2}-q},
\end{gathered}
$$

and $\chi^{\prime}(1)>D(n-1)$, whence $\chi(1)>D(n)$. Assume that $n \leq \mu_{b}$. Then $\left(\begin{array}{l}\lambda \\ \mu\end{array}\right)=$ $\left(\begin{array}{c}0,1,2 \ldots, a-2, a-1 \\ 1,2, \ldots, a-2, n\end{array}\right)$ with $n=\mu_{b} \geq a$ and $b=a-1$. If $a=3$, then 


$$
\begin{aligned}
& \left(\begin{array}{l}
\lambda \\
\mu
\end{array}\right)=\left(\begin{array}{ccc}
0 & 1 & 2 \\
1 & n
\end{array}\right) \text {, and } \chi(1)>D(n) \text {. Assume } a \geq 4 \text {. In this case } \\
& \chi(1) / \chi^{\prime}(1)=\prod_{i=0}^{a-1} \frac{q^{n}+q^{i}}{q^{n-1}+q^{i}} \cdot \prod_{i=1}^{a-2} \frac{q^{n}-q^{i}}{q^{n-1}-q^{i}}=\frac{\left(q^{n}+1\right)\left(q^{n-1}-1\right)}{\left(q^{n-a}+1\right)\left(q^{n-a+1}-1\right)}>q^{2 a-3} \geq q^{5},
\end{aligned}
$$

and we are again done.

5) Here we suppose that $\mu_{1}=0$ and $\lambda=(1,2, \ldots, a)$. Then consider the character $\chi^{\prime}$ labelled by the symbol

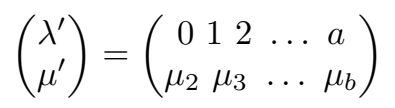

of the same rank $n$, but with the parameter $b^{\prime}=b-1$. If $\left(\begin{array}{c}\lambda^{\prime} \\ \mu^{\prime}\end{array}\right) \in \mathcal{L}_{n}$, then

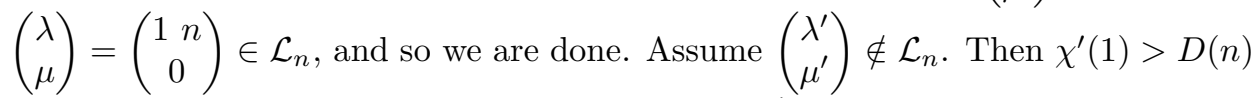
by the induction hypothesis (on $b)$. But $\chi(1)>\chi^{\prime}(1)$ (cf. [TZ1 p. 2122]), so we are done again.

Similarly, suppose that $\lambda_{1}=0, \mu=(1,2, \ldots, b)$, but $\lambda \neq(0,1, \ldots, a-1)$. Then there exists an $i \geq 2$ such that $\lambda_{i} \geq \lambda_{i-1}+2$. Consider the character $\chi^{\prime}$ labelled by the symbol $\left(\begin{array}{c}\lambda^{\prime} \\ \mu^{\prime}\end{array}\right)$ of rank $n-1$, where $\lambda^{\prime}=\left(\lambda_{1}, \ldots, \lambda_{i-1}, \lambda_{i}-1, \lambda_{i+1}, \ldots, \lambda_{a}\right)$, and $\mu^{\prime}=\mu$. If $\left(\begin{array}{l}\lambda^{\prime} \\ \mu^{\prime}\end{array}\right) \in \mathcal{L}_{n-1}$, then $\left(\begin{array}{l}\lambda \\ \mu\end{array}\right)=\left(\begin{array}{c}0 \\ 1\end{array}\right) \in \mathcal{L}_{n}$, and so we are done. So we may assume that $\chi^{\prime}(1)>D(n-1)$. Observe that $\chi(1) / \chi^{\prime}(1)>q^{2\left(n-\lambda_{i}\right)+1} / 2$; cf. [TZ1, p. 2123]. Therefore, if $n-\lambda_{i} \geq 3$, then $\chi(1) / \chi^{\prime}(1)>q^{6}$ and we are done. Assume $n-\lambda_{i} \leq 2$. If $i \leq a-1$, then the last condition implies that $i=a-1$ and $\left(\lambda_{a-1}, \lambda_{a}\right)=(n-2, n-1)$, whence

$$
\begin{aligned}
\frac{\chi(1)}{\chi^{\prime}(1)} & =\frac{q^{2 n}-1}{q^{2 \lambda_{i}}-1} \cdot \frac{q^{\lambda_{a}}-q^{\lambda_{i}}}{q^{\lambda_{a}}-q^{\lambda_{i}-1}} \cdot \prod_{i^{\prime}<i} \frac{q^{\lambda_{i}}-q^{\lambda_{i^{\prime}}}}{q^{\lambda_{i}-1}-q^{\lambda_{i^{\prime}}}} \cdot \prod_{j} \frac{q^{\lambda_{i}}+q^{\mu_{j}}}{q^{\lambda_{i}-1}+q^{\mu_{j}}} \\
& \geq \frac{q^{2 n}-1}{q^{2 n-4}-1} \cdot \frac{q^{n-1}-q^{n-2}}{q^{n-1}-q^{n-3}} \cdot \frac{q^{n-2}-1}{q^{n-3}-1} \cdot \frac{q^{n-2}+q}{q^{n-3}+q}>q^{5},
\end{aligned}
$$

and so we are done. Now we may assume that $i=a$ and $\left(\lambda_{1}, \ldots, \lambda_{a-1}\right)=$ $(0,1, \ldots, a-2)$. If $a=2$, then $\left(\begin{array}{l}\lambda \\ \mu\end{array}\right)=\left(\begin{array}{c}0 \\ 1\end{array}\right) \in \mathcal{L}_{n}$, so we may also assume that $a \geq 3$. If $n-\lambda_{a} \geq 1$, then

$$
\chi(1) / \chi^{\prime}(1)=\frac{q^{2 n}-1}{q^{2 \lambda_{a}}-1} \cdot \prod_{i^{\prime}=0}^{a-2} \frac{q^{\lambda_{a}}-q^{i^{\prime}}}{q^{\lambda_{a}-1}-q^{i^{\prime}}} \cdot \prod_{j=1}^{b} \frac{q^{\lambda_{a}}+q^{j}}{q^{\lambda_{a}-1}+q^{j}}>q^{5},
$$

and we are again done. Assume $n \leq \lambda_{a}$. In this case $n=\lambda_{a} \geq a$. If $a=3,4$ then

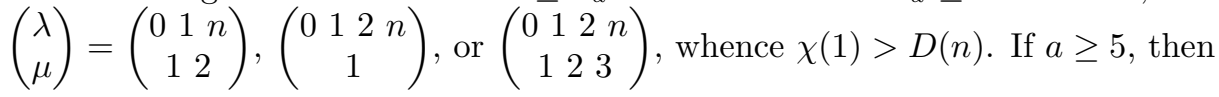

$$
\chi(1) / \chi^{\prime}(1) \geq \frac{q^{n}-1}{q^{n-1}-1} \cdot \frac{q^{n}-q}{q^{n-1}-q} \cdot \frac{q^{n}-q^{2}}{q^{n-1}-q^{2}} \cdot \frac{q^{n}-q^{3}}{q^{n-1}-q^{3}} \cdot \frac{q^{n}+q}{q^{n-1}+q}>q^{5},
$$

and we are again done. 
6) Finally, we consider the case where $\left(\begin{array}{l}\lambda \\ \mu\end{array}\right)=\left(\begin{array}{c}0,1,2, \ldots, a-1 \\ 1,2, \ldots, b\end{array}\right)$. Consider the character $\chi^{\prime}$ labelled by the symbol $\left(\begin{array}{c}\lambda^{\prime} \\ \mu^{\prime}\end{array}\right)=\left(\begin{array}{c}0,1,2, \ldots, a-2 \\ 1,2, \ldots, b-1\end{array}\right)$ of rank $n-1$. Observe that $\left(\begin{array}{c}\lambda^{\prime} \\ \mu^{\prime}\end{array}\right) \notin \mathcal{L}_{n-1}$, whence $\chi^{\prime}(1)>D(n-1)$. On the other hand, $\chi(1) / \chi^{\prime}(1)=q^{a+b-2}\left(q^{2 n}-1\right) /\left(q^{b}-1\right)\left(q^{a-1}+1\right)>\left(q^{2 n}-1\right) / q$, so $\chi(1)>D(n)$ as desired.

(B) We proceed by induction on $n$. The case $n=1$ is trivial. The case $n=2$ can be checked using $[\mathrm{E}$, and the case $(n, q)=(3,2)$ follows from Atlas. Hence for the induction step we may assume that $n \geq 3$ and $(n, q) \neq(3,2)$. Assume that $\chi$ is labelled by $\left((s), \chi_{u}\right)$, where $C:=C_{G}(s)$ is not equal to $G$. Set $E(n):=$ $\left(q^{2 n}-1\right)\left(q^{2 n-2}-1\right) /\left(q^{2}-1\right)(q+1)$.

1) One can show that $C=D \times S p_{2 m}(q)$, where $D \simeq \prod_{i=1}^{t} G L_{a_{i}}^{\alpha_{i}}\left(q^{k_{i}}\right), a_{i}, k_{i} \in \mathbb{N}$, $\alpha_{i}= \pm, m<n$, and $m+\sum_{i=1}^{t} k_{i} a_{i}=n$. Here we consider the case $m \geq 1$. Clearly, $\left(S p_{2 n-2 m}(q): D\right)_{2^{\prime}}$ is at least the smallest nonunipotent degree of $S p_{2 n-2 m}(q)$, which is $\left(q^{2 n-2 m}-1\right) /(q+1)$ by induction hypothesis. Hence

$$
\begin{aligned}
\chi(1) \geq(G: C)_{2^{\prime}}= & \left(G:\left(S p_{2 n-2 m}(q) \times S p_{2 m}(q)\right)\right)_{2^{\prime}} \cdot\left(S p_{2 n-2 m}(q): D\right)_{2^{\prime}} \\
& \geq \frac{q^{2 n-2 m}-1}{q+1} \cdot \prod_{i=1}^{m} \frac{q^{2(n-i+1)}-1}{q^{2 i}-1} .
\end{aligned}
$$

In particular, if $1 \leq m \leq n-2$, then $\chi(1) \geq E(n)$. Assume $m=n-1$. Then $D \simeq \mathbb{Z}_{q-\beta}$ with $\beta= \pm$. Observe that there are exactly $q / 2-1$, resp. $q / 2, G$ conjugacy classes of such elements $s$ for $\beta=+$, resp. for $\beta=-$. If $\chi_{u}(1)=1$, then $\chi(1)=\left(q^{2 n}-1\right) /(q-\beta)$. Assume $\chi_{u}(1)>1$. Then

$$
\chi_{u}(1) \geq\left(q^{n-1}-1\right)\left(q^{n-1}-q\right) / 2(q+1)
$$

by [LaS]. It follows that $\chi(1) \geq E(n)$ if $n \geq 3$ and $q \geq 4$, or if $n \geq 4$ and $D=\mathbb{Z}_{q-1}$. Assume that $n \geq 4$ and $(q, D)=\left(2, \mathbb{Z}_{q+1}\right)$. According to part $(\mathrm{A})$, either $\chi_{u}(1)=\left(q^{n-1}-\gamma\right)\left(q^{n-1}-\gamma q\right) / 2(q+1)$ for some $\gamma= \pm$ (and there is exactly one such a character for each $\gamma)$ or $\chi_{u}(1)>\left(q^{2 n-2}-1\right) /\left(q^{2}-1\right)$. In the former case (B5) holds, and in the latter case $\chi(1)>E(n)$.

Now we may assume that $m=0$; in particular, $C=D$.

2) Assume $n=3$; in particular, $q \geq 4$. If $D \neq G L_{3}^{\gamma}(q)$, then $\chi(1) \geq E(n)$. So $D=G L_{3}^{\gamma}(q)$. In this case, if $\chi_{u}(1)>1$, then $\chi_{u}(1)>q+1$ and so $\chi(1)>E(n)$. If $\chi_{u}(1)=1$, then (B3) holds.

Assume $n=4$. If $D \neq G L_{4}^{\gamma}(q)$, then $\chi(1) \geq E(n)$. So $D=G L_{4}^{\gamma}(q)$. In this case, if $\chi_{u}(1)>1$, then $\chi_{u}(1)>q+1$ and so $\chi(1)>E(n)$. If $\chi_{u}(1)=1$, then (B3) holds.

Assume $n \geq 5$. If $D \neq G U_{n}(q)$, then one can check that $\chi(1) \geq E(n)$. So $D=G U_{n}(q)$. In this case, if $\chi_{u}(1)>1$ or if $n \geq 6$, then again $\chi(1)>E(n)$. If $\chi_{u}(1)=1$ and $n=5$, then (B4) holds.

(C) We have completed the proof of Theorem 6.1, except that we have not identified the set $\left\{\chi^{\lambda, \mu} \mid\left(\begin{array}{l}\lambda \\ \mu\end{array}\right) \in \mathcal{L}_{n}\right\}$ with $\left\{1_{G}, \alpha_{n}, \beta_{n}, \rho_{n}^{1}, \rho_{n}^{2}\right\}$ and the set of $q-1$ non-unipotent characters of degree $\left(q^{2 n}-1\right) /(q \pm 1)$ with $\left\{\tau_{n}^{i} \mid 1 \leq i \leq q / 2-1\right\} \cup\left\{\zeta_{n}^{i} \mid\right.$ $1 \leq i \leq q / 2\}$. If $(n, q)=(3,2)$ or $(4,2)$, then this identification can be established 
using [Atlas]. Assume $n \geq 3$ and $(n, q) \neq(3,2),(4,2)$. Then these characters are exactly those of degree $\leq\left(q^{2 n}-1\right) /(q-1)$, and this identification follows immediately by degree comparison.

Theorem 6.1 immediately yields the following bound result:

Corollary 6.2. Let $G=S p_{2 n}(q)$ with $n \geq 3$ and $(n, q) \neq(3,2)$. Let

$$
D(n, q):= \begin{cases}(q-1)\left(q^{2}+1\right)\left(q^{3}-1\right), & n=3, \\ \left(q^{2 n}-1\right)\left(q^{n-1}-1\right)\left(q^{n-1}-q^{2}\right) / 2\left(q^{4}-1\right), & n \geq 4 .\end{cases}
$$

Then for any $\chi \in \operatorname{Irr}(G)$, either $\chi(1) \geq D(n, q)$, or $\chi$ is one of the $q+4$ characters $1_{G}, \alpha_{n}, \beta_{n}, \rho_{n}^{1}, \rho_{n}^{2}, \zeta_{n}^{i}, \tau_{n}^{i}$.

Notice that $D(n, q)=\frac{1}{2} q^{4 n-6} \cdot\left(1+q^{-4}+O\left(q^{-5}\right)\right)$.

Corollary 6.3. Let $G=S p_{2 n}(q)$ with $n \geq 3$ and $(n, q) \neq(3,2)$. The semisimple characters labelled by $s=\operatorname{diag}\left(\delta^{j}, \delta^{-j}, I_{2 n-2}\right)$ with $1 \leq j \leq q / 2-1$ are exactly the characters $\tau_{n}^{i}$. The semisimple characters labelled by $s \in G$ conjugate to $\operatorname{diag}\left(\xi^{j}, \xi^{-j}, I_{2 n-2}\right)$ with $1 \leq j \leq q / 2$ are exactly the characters $\zeta_{n}^{i}$.

Proof. Degree comparison using Theorem 6.1(B).

\section{Dual Pairs and BRANChing RUles For Weil Representations}

7.1. The dual pair $S p_{2 n}(q) \times O_{2}^{-}(q)$. We recall the construction [T] of the dual pair $S p_{2 n}(q) \times O_{2}^{-}(q)$ in characteristic 2 . Let $U=\mathbb{F}_{q^{2}}^{n}=\left\langle a_{1}, \ldots, a_{n}\right\rangle_{\mathbb{F}_{q^{2}}}$ be endowed with standard Hermitian form $\circ$ (that is, semilinear on the first component and that has $I_{n}$ as a Gram matrix in the given basis). Let $\bar{\alpha}=\alpha^{q}$ for any $\alpha \in \mathbb{F}_{q^{2}}$, and $\bar{u}=\sum_{i=1}^{n} \bar{\alpha}_{i} a_{i}$ for any $u=\sum_{i=1}^{n} \alpha_{i} a_{i} \in U$. We can define two nondegenerate symplectic forms, one on the $\mathbb{F}_{q}$-space $U:(u, v)=u \circ v+v \circ u$, and another on the $\mathbb{F}_{2}$-space $U:\langle u, v\rangle=\operatorname{tr}_{\mathbb{F}_{q} / \mathbb{F}_{2}}((u, v))$. Next we consider

$$
E=\mathbb{C}^{q^{2^{n}}}=\left\langle e_{u} \mid u \in U\right\rangle_{\mathbb{C}} .
$$

The dual pair $S p_{2 n}(q) \times O_{2}^{-}(q)$ will be defined inside $G L(E)$. For each $v \in U$ consider the following elements of $G L(E)$ :

$$
f_{v}: e_{u} \mapsto(-1)^{\langle u, v\rangle} e_{u}, \quad t_{v}: e_{u} \mapsto e_{u+v} .
$$

Then $P:=\left\langle f_{v}, t_{v} \mid v \in U\right\rangle \simeq 2^{1+4 n f}$ is an extraspecial 2-subgroup of $G L(E)$. Let

$$
\mathcal{N}:=\left\{X \in G L(E) \mid X P X^{-1}=P, \operatorname{det}(X)= \pm 1\right\} .
$$

It is known that $\mathcal{N} / Z(\mathcal{N}) P \geq \Omega_{4 n f}^{+}(2)$ contains canonical subgroups $\bar{H} \simeq G U_{2 n}(q)$ and $\bar{S} \simeq S p_{2 n}(q)$. In particular, if $g \in S p_{2 n}(q)=S p(U,(\cdot, \cdot))$, then $g f_{u} g^{-1}=f_{g(u)}$, $g t_{u} g^{-1}=t_{g(u)}$. Let $\widehat{H}$, resp. $\widehat{S}$, be the complete inverse image of $\bar{H}$, resp. of $\bar{S}$, in $\mathcal{N}$. Fix $\theta \in \mathbb{F}_{q^{2}}^{\bullet}$ of order $q+1$, and consider the following two elements of $\mathcal{N}$ :

$$
\vartheta: e_{u} \mapsto e_{\theta u}, \quad j: e_{u} \mapsto e_{\bar{u}} .
$$

Clearly, $D:=\langle\boldsymbol{\vartheta}, \boldsymbol{j}\rangle \simeq O_{2}^{-}(q)$. It is shown in [T] that $C_{\widehat{S}}(\boldsymbol{\vartheta}, \boldsymbol{j})=Z(\mathcal{N}) \times S$, where

$$
S=C_{\widehat{S}}(\boldsymbol{\vartheta}, \boldsymbol{j})^{(\infty)} \simeq S p_{2 n}(q),
$$

provided that $(n, q) \neq(1,2),(2,2),(3,2),(1,4)$. The subgroup $S \times D$ is the desired dual pair $S p_{2 n}(q) \times O_{2}^{-}(q)$. On the other hand, under the same assumption on $(n, q), C_{\widehat{H}}(\boldsymbol{\vartheta})=Z(\mathcal{N}) \times H$, where $H=O^{2}\left(C_{\widehat{H}}(\boldsymbol{\vartheta})\right) \simeq G U_{2 n}(q)$. The subgroup 
$H \times\langle\boldsymbol{\vartheta}\rangle$ forms the dual pair $G U_{2 n}(q) \times G U_{1}(q)$; these two dual pairs $H \times\langle\boldsymbol{\vartheta}\rangle$ and $S \times D$ form what is called a pair of see-saw dual pairs in the terminology of Kudla $\mathrm{Ku}$.

Let $\omega_{n}$ denote the character of $S \times D$ acting on $E$. It is also shown in [T] that $\left.\omega_{n}\right|_{S}=\zeta_{n}$, and moreover one can label the irreducible characters of $D$ as $\beta=1_{D}$, $\alpha$ of degree 1 , and $\nu_{i}, 1 \leq i \leq q / 2$, of degree 2 such that

$$
\left.\omega_{n}\right|_{S \times D}=\alpha_{n} \otimes \alpha+\beta_{n} \otimes \beta+\sum_{i=1}^{q / 2} \zeta_{n}^{i} \otimes \nu_{i} .
$$

Assume $q \geq 8$, and let $k, l \in \mathbb{N}$ be such that $k+l=n$. We can repeat the above construction but with $n$ replaced throughout by $k$, resp. by $l$, and subscript 1 , resp. 2, attached to all letters $U, E, P, \mathcal{N}, S, D, \boldsymbol{\vartheta}$, and $\boldsymbol{j}$. Thus we get the dual pair $S_{1} \times D_{1} \simeq S p_{2 k}(q) \times O_{2}^{-}(q)$ inside $G L\left(E_{1}\right)$ with character $\omega_{k}$, and the dual pair $S_{2} \times D_{2} \simeq S p_{2 l}(q) \times O_{2}^{-}(q)$ inside $G L\left(E_{2}\right)$ with character $\omega_{l}$. Now we can identify $U$ with $U_{1} \oplus U_{2}$. This in turn identifies $E$ with $E_{1} \otimes E_{2}, P$ with $P_{1} \otimes P_{2}, \vartheta$ with $\boldsymbol{\vartheta}_{1} \otimes \boldsymbol{\vartheta}_{2}, \boldsymbol{j}$ with $\boldsymbol{j}_{1} \otimes \boldsymbol{j}_{2}$. This identification also embeds $\mathcal{N}_{1} \otimes \mathcal{N}_{2}$ in $\mathcal{N}, \bar{S}_{1} \times \bar{S}_{2}$ in $\bar{S}$, and $\widehat{S}_{1} \times \widehat{S}_{2}$ in $\widehat{S}$. Suppose $g_{1} \in S_{1}$ and $g_{2} \in S_{2}$. Then $g_{1} \otimes g_{2}$ centralizes both $\vartheta$ and $\boldsymbol{j}$, and belongs to $\widehat{S}$. It follows by (12) that

$$
S_{1} \times S_{2}=\left(S_{1} \times S_{2}\right)^{(\infty)}<C_{\widehat{S}}(\boldsymbol{\vartheta}, \boldsymbol{j})^{(\infty)}=S .
$$

Moreover, if $g:=g_{1} \otimes g_{2}, x:=\boldsymbol{\vartheta}^{a} \boldsymbol{j}^{b}$ (for some $a, b \in \mathbb{Z}$ ), then

$$
x g=\left(\boldsymbol{\vartheta}_{1} \otimes \boldsymbol{\vartheta}_{2}\right)^{a}\left(\boldsymbol{j}_{1} \otimes \boldsymbol{j}_{2}\right)^{b}\left(g_{1} \otimes g_{2}\right)=\boldsymbol{\vartheta}_{1}^{a} \boldsymbol{j}_{1}^{b} g_{1} \otimes \boldsymbol{\vartheta}_{2}^{a} \boldsymbol{j}_{2}^{b} g_{2}=x_{1} g_{1} \otimes x_{2} g_{2},
$$

where $x_{1}=\boldsymbol{\vartheta}_{1}^{a} \boldsymbol{j}_{1}^{b}, x_{2}=\boldsymbol{\vartheta}_{2}^{a} \boldsymbol{j}_{2}^{b}$. Taking trace and identifying $x_{1}, x_{2}$ with $x$ (which causes no loss in computing $\omega_{n}, \omega_{k}$, and $\omega_{l}$ ) we obtain

Lemma 7.1. Let $q \geq 8$ and let $S p_{2 k}(q) \times S p_{2 l}(q)$ be a standard subgroup of $S p_{2 n}(q)$ with $k+l=n$. Then $\omega_{n}(x g)=\omega_{k}\left(x g_{1}\right) \cdot \omega_{l}\left(x g_{2}\right)$ for any $x \in O_{2}^{-}(q)$ and any $g=\left(g_{1}, g_{2}\right) \in S p_{2 k}(q) \times S p_{2 l}(q)$.

Proposition 7.2. Let $S=S p_{2 n}(q)$ with $n \geq 3$ and let $H=S p_{2 n-2}(q)$ be a standard subgroup of $S$. Then

$$
\begin{gathered}
\left.\alpha_{n}\right|_{H}=q \alpha_{n-1}+(q-1) \sum_{k=1}^{q / 2} \zeta_{n-1}^{k},\left.\beta_{n}\right|_{H}=q \beta_{n-1}+(q-1) \sum_{k=1}^{q / 2} \zeta_{n-1}^{k}, \\
\left.\zeta_{n}^{i}\right|_{H}=\zeta_{n-1}^{i}+(q-1)\left(2 \sum_{k=1}^{q / 2} \zeta_{n-1}^{k}+\alpha_{n-1}+\beta_{n-1}\right)
\end{gathered}
$$

for $1 \leq i \leq q / 2$.

Proof. 1) The standard embedding of $H$ in $S$ can be written as $h \in H \mapsto g=$ $\operatorname{diag}(1,1, h) \in S$. In the notation of $\S 3$ it follows that $\operatorname{dim}_{\mathbb{F}_{q^{2}}} \operatorname{Ker}\left(g-\xi^{j}\right)$ equals $2+\operatorname{dim}_{\mathbb{F}_{q^{2}}} \operatorname{Ker}\left(h-\xi^{j}\right)$ if $j=0$ and $\operatorname{dim}_{\mathbb{F}_{q^{2}}} \operatorname{Ker}\left(h-\xi^{j}\right)$ if $j \neq 0$. Hence (4) implies that

$$
\left.\zeta_{n}^{i}\right|_{H}=\sum_{j \neq i, k \neq j} \zeta_{n-1}^{k}=q \zeta_{n-1}^{i}+(q-1) \sum_{k \neq i} \zeta_{n-1}^{k}=\zeta_{n-1}^{i}+(q-1) \sum_{k=0}^{q} \zeta_{n-1}^{k}
$$


for any $i=0,1, \ldots, q$. In particular, we get the desired formula for $i \neq 0$, since $\zeta_{n-1}^{0}=\alpha_{n-1}+\beta_{n-1}$. In order to prove the formulae for $\left.\alpha_{n}\right|_{H}$ and $\left.\beta_{n}\right|_{H}$, it suffices to prove one of them, since we have just shown that

$$
\left.\zeta_{n}^{0}\right|_{H}=q\left(\alpha_{n-1}+\beta_{n-1}\right)+2(q-1) \sum_{k=1}^{q / 2} \zeta_{n-1}^{k} .
$$

2) Here we assume that $q \geq 8$. According to (13)), $\beta_{n}$ is just the $S$-character of the $1_{D}$-homogeneous component of $\omega_{n}$, whence

$$
\beta_{n}(g)=\frac{1}{2(q+1)} \sum_{x \in D} \omega_{n}(x g) .
$$

If $g=\operatorname{diag}(1,1, h)=1 \otimes h$ as in 1$)$, then $\omega_{n}(x g)=\omega_{1}(x) \cdot \omega_{n-1}(x h)$ by Lemma 7.1. Moreover, if $0 \leq a \leq q$ and $b=0,1$, then $\omega_{1}\left(\boldsymbol{\vartheta}^{a} \boldsymbol{j}^{b}\right)$ equals $q^{2}$, resp. $q$, or 1 , provided that $(a, b)=(0,0)$, resp. $b=1$, or $(a, b)=(\neq 0,0)$. It follows that

$$
\begin{aligned}
& \beta_{n}(g)=\left(q^{2} \omega_{n-1}(h)+\sum_{a=1}^{q} \omega_{n-1}\left(\boldsymbol{\vartheta}^{a} h\right)+q \sum_{a=0}^{q} \omega_{n-1}\left(\boldsymbol{\vartheta}^{a} \boldsymbol{j} h\right)\right) / 2(q+1), \\
& \beta_{n-1}(h)=\left(\omega_{n-1}(h)+\sum_{a=1}^{q} \omega_{n-1}\left(\boldsymbol{\vartheta}^{a} h\right)+\sum_{a=0}^{q} \omega_{n-1}\left(\boldsymbol{\vartheta}^{a} \boldsymbol{j} h\right)\right) / 2(q+1) .
\end{aligned}
$$

Therefore

$$
\begin{gathered}
\beta_{n}(g)-q \beta_{n-1}(h)=\frac{q-1}{2(q+1)}\left(q \omega_{n-1}(h)-\sum_{a=1}^{q} \omega_{n-1}\left(\boldsymbol{\vartheta}^{a} h\right)\right) \\
=\frac{q-1}{2(q+1)}\left((q+1) \omega_{n-1}(h)-\sum_{a=0}^{q} \omega_{n-1}\left(\boldsymbol{\vartheta}^{a} h\right)\right)=\frac{q-1}{2} \cdot \omega_{n-1}(h)-\frac{q-1}{2} \cdot \zeta_{n-1}^{0}(h) \\
=\frac{q-1}{2}\left(\alpha_{n-1}+\beta_{n-1}+2 \sum_{k=1}^{q / 2} \zeta_{n-1}^{k}\right)(h)-\frac{q-1}{2}\left(\alpha_{n-1}+\beta_{n-1}\right)(h) \\
=(q-1) \sum_{k=1}^{q / 2} \zeta_{n-1}^{k}(h),
\end{gathered}
$$

and so we are done.

3) Now we consider the case $q=2,4$; in particular, $q+1$ is prime. By (14), there are nonnegative integers $a, b, c_{i}$ with $a, b \leq q$ and $\sum_{i=1}^{q / 2} c_{i} \leq q(q-1)$ such that $\left.\beta_{n}\right|_{H}=a \alpha_{n-1}+b \beta_{n-1}+\sum_{i=1}^{q / 2} c_{i} \zeta_{n-1}^{i}$. Since the degrees of $\beta_{n}, \beta_{n-1}$, and $\zeta_{n-1}^{i}$ are divisible by $q^{n-1}+1$, so is $a \alpha_{n-1}(1)$. But $0 \leq a \leq q$, hence $a=0$. Comparing the degree we see $q(q-b)\left(q^{n-2}+1\right)=\left(2 \sum_{i} c_{i}-q(q-1)\right)\left(q^{n-1}-1\right)$.

Claim that $b=q$ and $\sum_{i} c_{i}=q(q-1) / 2$. Indeed, the above equality implies that $(q-b)(q+1)$ is divisible by $q^{n-1}-1$. If $n \geq 4$, then it follows that $b=q$. If $n=3$ but $b \neq q$, then $b=1$ and $\sum_{i} c_{i}=q^{2} / 2$. However this would imply $\beta_{3}(t)=-\left(q^{4}-q^{3}+2 q^{2}-q\right) / 2$ for any transvection $t \in H$, contrary to [Lu].

We have shown that $\left.\beta_{n}\right|_{H}=q \beta_{n-1}+\sum_{i=1}^{q / 2} c_{i} \zeta_{n-1}^{i}$ with $\sum_{i} c_{i}=q(q-1) / 2$. Recall that we are assuming $q+1$ is prime, so $\Gamma:=\operatorname{Gal}(\mathbb{Q}(\xi) / \mathbb{Q})$ is cyclic. Formula (49) shows that $\Gamma$ acts transitively on the set $\left\{\zeta_{n-1}^{i} \mid 1 \leq i \leq q / 2\right\}$; indeed, the Galois automorphism $\xi \mapsto \xi^{k}$ sends $\zeta_{n-1}^{i}$ to $\zeta_{n-1}^{i k}$. On the other hand, $\beta_{n}$, as the 
only irreducible constituent of degree $\left(q^{n}+1\right)\left(q^{n}+q\right) / 2(q+1)$ of the rationalvalued character $\zeta_{n}$, is $\Gamma$-stable. Similarly, $\beta_{n-1}$ is $\Gamma$-stable. It therefore follows that $c_{1}=\ldots=c_{q / 2}=q-1$, as stated.

In what follows we need the character values at some particular elements of $G$; namely a transvection (equivalently, a long-root element) $\boldsymbol{t}:=\operatorname{diag}\left(\left(\begin{array}{ll}1 & 1 \\ 0 & 1\end{array}\right), I_{2 n-2}\right)$, a short-root element $s:=\operatorname{diag}\left(\left(\begin{array}{llll}1 & 0 & 0 & 1 \\ 0 & 1 & 0 & 0 \\ 0 & 1 & 1 & 0 \\ 0 & 0 & 0 & 1\end{array}\right), I_{2 n-4}\right)$, a double-transvection $\boldsymbol{d}:=\operatorname{diag}\left(\left(\begin{array}{ll}1 & 1 \\ 0 & 1\end{array}\right),\left(\begin{array}{ll}1 & 1 \\ 0 & 1\end{array}\right), I_{2 n-4}\right)$, and semisimple elements

$$
\boldsymbol{c}_{j}:=\operatorname{diag}\left(\delta^{j}, \delta^{-j}, 1, \ldots, 1\right), \quad 1 \leq j \leq(q-2) / 2,
$$

where all matrices are written with respect to the basis

$$
\left(e_{1}, f_{1}, e_{2}, f_{2}, e_{3}, f_{3}, \ldots, e_{n}, f_{n}\right) .
$$

If we embed the corresponding elements in a standard subgroup $S p_{4}(q)$, then $\boldsymbol{t}, \boldsymbol{s}$, and $\boldsymbol{d}$ belong to classes $A_{2}, A_{31}$, and $A_{32}$ (in the notation of [E]), respectively.

Corollary 7.3. Let $G=S p_{2 n}(q)$ with $n \geq 2$ and let $1 \leq i \leq q / 2,1 \leq j \leq(q-2) / 2$. Then

$$
\begin{gathered}
\alpha_{n}(\boldsymbol{t})=\beta_{n}(\boldsymbol{t})=-\frac{q^{2 n-1}-q}{2(q+1)}, \zeta_{n}^{i}(\boldsymbol{t})=-\frac{q^{2 n-1}+1}{q+1}, \\
\alpha_{n}(\boldsymbol{s})=\frac{q^{2 n-2}-q^{n+1}-q^{n}+q}{2(q+1)}, \beta_{n}(\boldsymbol{s})=\frac{q^{2 n-2}+q^{n+1}+q^{n}+q}{2(q+1)}, \\
\zeta_{n}^{i}(\boldsymbol{s})=\frac{q^{2 n-2}-1}{q+1}, \\
\alpha_{n}(\boldsymbol{d})=\beta_{n}(\boldsymbol{d})=\frac{q^{2 n-2}+q}{2(q+1)}, \zeta_{n}^{i}(\boldsymbol{d})=\frac{q^{2 n-2}-1}{q+1}, \\
\alpha_{n}\left(\boldsymbol{c}_{j}\right)=\frac{q^{2 n-2}-q^{n}-q^{n-1}+q}{2(q+1)}, \beta_{n}\left(\boldsymbol{c}_{j}\right)=\frac{q^{2 n-2}+q^{n}+q^{n-1}+q}{2(q+1)}, \\
\zeta_{n}^{i}\left(\boldsymbol{c}_{j}\right)=\frac{q^{2 n-2}-1}{q+1} .
\end{gathered}
$$

Proof. The character values of $\zeta_{n}^{i}$ for $0 \leq i \leq q / 2$ are computed using (4). In particular, we know the character values of $\zeta_{n}^{0}=\alpha_{n}+\beta_{n}$. We will deal with $\alpha_{n}$ and $\beta_{n}$ by induction on $n$. The induction base $n=2$ can be checked using [E]. For the induction step assume $n \geq 3$. We may also assume that $\boldsymbol{t}, \boldsymbol{s}, \boldsymbol{d}, \boldsymbol{c}_{j}$ are contained in a standard subgroup $H \simeq S p_{2 n-2}(q)$. By Proposition 7.2 . $\left(\beta_{n}-\alpha_{n}\right)(x)=$ $q\left(\beta_{n-1}-\alpha_{n-1}\right)(x)$ for any $x \in\left\{\boldsymbol{t}, \boldsymbol{s}, \boldsymbol{d}, \boldsymbol{c}_{j}\right\}$. Since we know $\alpha_{n}+\beta_{n}$ and we know $\left(\beta_{n-1}-\alpha_{n-1}\right)(x)$ by induction, the stated formulae follow easily.

Recall that for any $Q_{1}$-character $\lambda \in \mathcal{O}_{2}^{\varepsilon}, K_{\lambda}:=\operatorname{Stab}_{P_{1}}(\lambda)=Q_{1}: I_{\lambda}$, where $I_{\lambda}:=\operatorname{Stab}_{L_{1}}(\lambda)$ equals $J_{\lambda}:=\operatorname{Stab}_{L_{1}^{\prime}}(\lambda) \simeq O_{2 n-2}^{\varepsilon}(q)$. Let $\hat{\kappa}$ be the character of $K_{\lambda}$, where $\left.\hat{\kappa}\right|_{Q_{1}}=\lambda$ and $\left.\hat{\kappa}\right|_{I_{\lambda}}=\kappa$. In what follows, the $P_{1}$-module $\boldsymbol{B}_{\varepsilon}$ is defined to be $\operatorname{Ind}_{K_{\lambda}}^{P_{1}}(\hat{\kappa})$. In the next statement, we will consider $\alpha_{n-1} \otimes 1_{T_{1}}$ as a character of $L_{1}=L_{1}^{\prime} \times T_{1}$, and then inflate it to $P_{1}=Q_{1} L_{1}$. Similarly for $\beta_{n-1} \otimes 1_{T_{1}}, \zeta_{n-1}^{i} \otimes 1_{T_{1}}$. 
Proposition 7.4. Let $G=S p_{2 n}(q)$ with $n \geq 3,(n, q) \neq(3,2),(4,2)$, and let $1 \leq i \leq q / 2$. Then

$\left.\alpha_{n}\right|_{P_{1}}=\alpha_{n-1} \otimes 1_{T_{1}}+\boldsymbol{B}_{-},\left.\beta_{n}\right|_{P_{1}}=\beta_{n-1} \otimes 1_{T_{1}}+\boldsymbol{B}_{+},\left.\zeta_{n}^{i}\right|_{P_{1}}=\zeta_{n-1}^{i} \otimes 1_{T_{1}}+\boldsymbol{B}_{+}+\boldsymbol{B}_{-}$.

Proof. 1) Let $\phi \in\left\{\alpha_{n}, \beta_{n}, \zeta_{n}^{i}\right\}$ and let $V$ be a module affording $\phi$. By Corollary 4.2. $\phi$ satisfies $\left(\mathcal{W}_{2}^{-}\right)$, whence $\phi$ satisfies $\left(\mathcal{W}_{1}\right)$ by Proposition 5.7. It follows that $\phi$ satisfies the conclusion of Theorem [5.11(i). Thus there are nonnegative integers $c, d$ such that $\left.V\right|_{P_{1}}=C_{V}\left(Q_{1}\right)+c \boldsymbol{B}_{+}+d \boldsymbol{B}_{-}$and moreover $(c, d) \neq(0,0)$. By Proposition 7.2, there are nonnegative integers $a, b, e$ such that

$$
\operatorname{dim}\left(C_{V}\left(Q_{1}\right)\right)=a \alpha_{n-1}(1)+b \beta_{n-1}(1)+e \zeta_{n-1}^{1}(1) .
$$

First we consider the case $\phi=\alpha_{n}$. Observe that $\alpha_{n}(1)=\alpha_{n-1}(1)+\boldsymbol{B}_{-}(1)<$ $2 \boldsymbol{B}_{-}(1)<\boldsymbol{B}_{-}(1)+\boldsymbol{B}_{+}(1)$, and $\boldsymbol{B}_{+}(1)<\alpha_{n}(1)<\alpha_{n-1}(1)+\boldsymbol{B}_{+}(1)$ if $n \geq 4$ and $(n, q) \neq(4,2)$, and $\alpha_{n}(1)<\boldsymbol{B}_{+}(1)$ if $n=3$. It follows that $(c, d)=(0,1)$ and $C_{V}\left(Q_{1}\right)$ affords the $L_{1}^{\prime}$-character $\alpha_{n-1}$. Thus we have proved the desired formula for $\left.\alpha_{n}\right|_{P_{1}^{\prime}}$. In particular,

$$
\left.\boldsymbol{B}_{-}\right|_{L_{1}^{\prime}}=(q-1)\left(\alpha_{n-1}+\sum_{k=1}^{q / 2} \zeta_{n-1}^{k}\right)
$$

Next we consider the case $\phi=\beta_{n}$. Since $\beta_{n}(1)<2 \boldsymbol{B}_{-}(1)<\boldsymbol{B}_{+}(1)+\boldsymbol{B}_{-}(1)$, $\{c, d\}=\{0,1\}$. Assume $d=1$. Then $\left.\beta_{n}\right|_{L_{1}^{\prime}}$ contains $\alpha_{n-1}$ by (16), contrary to Proposition 7.2. Hence $(c, d)=(1,0)$. Now $\operatorname{dim}\left(C_{V}\left(Q_{1}\right)\right)=\beta_{n-1}(1)$, whence (15) implies that $(a, b, e)=(0,1,0)$ (since $n \geq 3$ and $(n, q) \neq(3,2),(4,2))$. Thus we have proved the desired formula for $\left.\beta_{n}\right|_{P_{1}^{\prime}}$. In particular,

$$
\left.\boldsymbol{B}_{+}\right|_{L_{1}^{\prime}}=(q-1)\left(\beta_{n-1}+\sum_{k=1}^{q / 2} \zeta_{n-1}^{k}\right)
$$

Now we consider the case $\phi=\zeta_{n}^{i}$. Applying Proposition 7.2 and (16), (17) to $\left.\zeta_{n}^{i}\right|_{P_{1}}=C_{V}\left(Q_{1}\right)+c \boldsymbol{B}_{+}+d \boldsymbol{B}_{-}$, we get

$$
\begin{gathered}
\zeta_{n-1}^{i}+(q-1)\left(\alpha_{n-1}+\beta_{n-1}+2 \sum_{k=1}^{q / 2} \zeta_{n-1}^{k}\right) \\
=C_{V}\left(Q_{1}\right)+c(q-1)\left(\beta_{n-1}+\sum_{k=1}^{q / 2} \zeta_{n-1}^{k}\right)+d(q-1)\left(\alpha_{n-1}+\sum_{k=1}^{q / 2} \zeta_{n-1}^{k}\right) .
\end{gathered}
$$

Since all $\alpha_{n-1}, \beta_{n-1}$, and $\zeta_{n-1}^{k}$ are irreducible and distinct, we see that $c \leq 1$ and $d \leq 1$. If $(c, d)=(1,0)$, then $\alpha_{n-1}$ appears in $C_{V}\left(Q_{1}\right)$ but not in $\left[V, Q_{1}\right]$, contrary to Lemma 2.1. Similarly, $(c, d) \neq(0,1)$. Thus $c=d=1$, and $C_{V}\left(Q_{1}\right)=\zeta_{n-1}^{i}$ as $L_{1}^{\prime}$-module, and so we have proved the desired formula for $\left.\zeta_{n}^{i}\right|_{P_{1}^{\prime}}$.

2) Consider any $g \in P_{1} \backslash P_{1}^{\prime}$. Write $g=\left(\begin{array}{ccc}\delta^{j} & * & * \\ 0 & h & * \\ 0 & 0 & \delta^{-j}\end{array}\right)$ in the basis $\left(e_{1}, \ldots, e_{n}\right.$, $\left.f_{2}, \ldots, f_{n}, f_{1}\right)$, for some $\delta^{j} \neq 1$ and $h \in L_{1}^{\prime}$. For any $\lambda \in \mathcal{O}_{2}^{\varepsilon}, \operatorname{Stab}_{P_{1}}(\lambda)<P_{1}^{\prime}$, whence $\boldsymbol{B}_{+}(g)=\boldsymbol{B}_{-}(g)=0$. Therefore, in order to finish the proof of the proposition, it suffices to show that $\zeta_{n}^{i}(g)=\zeta_{n-1}^{i}(h)$ for any $i=0,1, \ldots, q / 2$. Indeed, for any $\xi^{k}$ we have $\delta^{ \pm j} \neq \xi^{k}$, and so direct computation shows that

$$
\left|\left\{v \in W \otimes_{\mathbb{F}_{q}} \mathbb{F}_{q^{2}} \mid g(v)=\xi^{k} v\right\}\right|=\left|\left\{w \in\left\langle e_{2}, \ldots, e_{n}, f_{2}, \ldots, f_{n}\right\rangle_{\mathbb{F}_{q^{2}}} \mid h(w)=\xi^{k} w\right\}\right|,
$$


i.e. $\operatorname{dim}_{\mathbb{F}_{q^{2}}} \operatorname{Ker}\left(g-\xi^{k}\right)=\operatorname{dim}_{\mathbb{F}_{q^{2}}} \operatorname{Ker}\left(h-\xi^{k}\right)$. Now (4) yields that $\zeta_{n}^{i}(g)=\zeta_{n-1}^{i}(h)$, as desired.

Corollary 7.5. Let $G=S p_{2 n}(q)$ with $n \geq 2$. Write $q+1=\ell^{a} r$ with $(r, \ell)=1$.

(i) Let $\varphi$ be any nontrivial composition factor of $\widehat{\alpha}_{n}, \widehat{\beta}_{n}$, or $\hat{\zeta}_{n}^{i}$. Then $\varphi$ is of form $\rho-a \cdot 1_{G}$, where $\rho \in\left\{\widehat{\alpha}_{n}, \widehat{\beta}_{n}, \hat{\zeta}_{n}^{j} \mid 1 \leq j \leq(r-1) / 2\right\}$, and $a=0$ or 1 . Moreover, $a=1$ if and only if $\ell \mid(q+1)$ and $\rho=\widehat{\beta}_{n}$.

(ii) The Brauer characters $\widehat{\alpha}_{n}, \widehat{\beta}_{n}, \hat{\zeta}_{n}^{j}$ with $1 \leq j \leq(r-1) / 2$ each have a unique nontrivial irreducible constituent, and these $(r+3) / 2$ constituents are paiwise distinct.

Proof. (i) The statement for $n=2$, resp. for $(n, q)=(3,2)$ and $(4,2)$, can be checked directly using [Wh1, resp. JJLPW]. We will proceed by induction on $n$ and assume $n \geq 3$ and $(n, q) \neq(3,2),(4,2)$.

1) It was established in the proof of [DT, Theorem 7.2] that

$$
\hat{\zeta}_{n}^{i}-\hat{\zeta}_{n}^{j}=\delta_{i, 0}-\delta_{j, 0},
$$

whenever $0 \leq i, j \leq q$ and $i \equiv j(\bmod r)$. Together with (5) and (6), (18) implies that any nontrivial composition factor of $\hat{\zeta}_{n}^{i}$ is a composition factor of $\widehat{\alpha}_{n}, \widehat{\beta}_{n}$, or $\hat{\zeta}_{n}^{j}$ with $1 \leq j \leq(r-1) / 2$.

2) Consider the semisimple character $\phi_{j}$ labelled by $s_{j} \in G$ conjugate to $\operatorname{diag}\left(\xi^{j}, \xi^{-j}, I_{2 n-2}\right)$ with $1 \leq j \leq(r-1) / 2$. Since the $\ell^{\prime}$-part $s_{j}^{\prime}$ of $s_{j}$ is nontrivial semisimple and $\phi_{j}(1)=\left(q^{2 n}-1\right) /(q+1)=\left(G: C_{G}\left(s_{j}^{\prime}\right)\right)_{2^{\prime}}$, [HM] Proposition 1] implies that $\widehat{\phi}_{j}$ is irreducible. Moreover, these $(r-1) / 2$ characters $\phi_{j}$ belong to $(r-1) / 2$ distinct nonunipotent $\ell$-blocks, since the $s_{j}^{\prime}$ are pairwise nonconjugate and nontrivial. On the other hand, by Corollary 6.3 $\left\{\phi_{j} \mid 1 \leq j \leq(r-1) / 2\right\} \subseteq\left\{\zeta_{n}^{i} \mid\right.$ $1 \leq i \leq q / 2\}$. The result of 1$)$ shows that there are at most $(r-1) / 2$ distinct irreducible Brauer characters of degree $\left(q^{2 n}-1\right) /(q+1)$ that can arise as composition factors of $\hat{\zeta}_{n}^{i}$. We conclude that the Brauer characters $\hat{\zeta}_{n}^{j}$ with $1 \leq j \leq(r-1) / 2$ are irreducible and pairwise distinct.

3) Since $\alpha_{n}$ meets the Landazuri-Seitz-Zalesskii bound $d(G), \widehat{\alpha}_{n}$ is irreducible. Next assume $\varphi$ is a nontrivial composition factor of $\widehat{\beta}_{n}$ and $\varphi \neq \widehat{\beta}_{n}$. Clearly, $\varphi$ cannot be trivial on $Q_{1}$. On the other hand, $\left.\beta_{n}\right|_{P_{1}}=\beta_{n-1} \otimes 1_{T_{1}}+\boldsymbol{B}_{+}$by Proposition 7.4, and $\widehat{\boldsymbol{B}}_{+}$is irreducible over $P_{1}$. It follows that $\left.\varphi\right|_{P_{1}}$ contains $\widehat{\boldsymbol{B}}_{+}$. Now any composition factor of $\widehat{\beta}_{n}-\varphi$ is trivial on $Q_{1}$ and therefore equal to $1_{G}$. Thus $\varphi=\widehat{\beta}_{n}-a \cdot 1_{G}$ with $a \geq 1$. Let $\psi$ be any $P_{1}$-composition factor of $\varphi-\widehat{\boldsymbol{B}}_{+}$. Then $\psi$ is trivial on $Q_{1}$ and $\left.\psi\right|_{L_{1}^{\prime}}$ is contained in $\widehat{\beta}_{n-1}$. By induction hypothesis and by the assumption on $\varphi,\left.\psi\right|_{L_{1}^{\prime}}$ equals $1_{L_{1}^{\prime}}$ or $\widehat{\beta}_{n-1}-1_{L_{1}^{\prime}}$. If the first possibility realizes for all such $\psi$, then we come to the conclusion that the Brauer character $\left.\widehat{\beta}_{n}\right|_{P_{1}}-\widehat{\boldsymbol{B}}_{+}$is trivial on $P_{1}$, contrary to Proposition 7.4. Hence the second possibility must occur for at least one such $\psi$, whence $\ell \mid(q+1)$ and $\varphi=\widehat{\beta}_{n}-1_{G}$.

Conversely, assume $\ell \mid(q+1)$. Then (6i) and (18) imply that $\widehat{\alpha}_{n}+\widehat{\beta}_{n}=\left.\hat{\zeta}_{n}^{0}\right|_{G}=$ $1_{G}+\hat{\zeta}_{n}^{r}$, whence $1_{G}$ must occur either in $\widehat{\alpha}_{n}$ or $\widehat{\beta}_{n}$. But $\widehat{\alpha}_{n}$ is irreducible, hence $1_{G}$ enters $\widehat{\beta}_{n}$ and the above discussion shows that $\widehat{\beta}_{n}-1_{G}$ is irreducible.

(ii) follows from (i) and degree comparison. 
7.2. The dual pair $S p_{2 n}(q) \times O_{2}^{+}(q)$. We recall the construction [T] of the dual pair $S p_{2 n}(q) \times O_{2}^{+}(q)$ in characteristic 2. Let $U=\mathbb{F}_{q}^{2 n}$ be endowed with standard symplectic form $(\cdot, \cdot)$. We will also consider the $\mathbb{F}_{2}$-symplectic form $\langle u, v\rangle=$ $\operatorname{tr}_{\mathbb{F}_{q} / \mathbb{F}_{2}}((u, v))$ on $U$, and let

$$
E=\mathbb{C}^{q^{2^{n}}}=\left\langle e_{u} \mid u \in U\right\rangle_{\mathbb{C}} .
$$

Clearly, $S:=S p_{2 n}(q)$ acts on $E$ via $g: e_{u} \mapsto e_{g(u)}$. Fix $\delta \in \mathbb{F}_{q}^{\bullet}$ of order $q-1$, and consider the following endomorphisms of $E$ :

$$
\delta: e_{u} \mapsto e_{\delta u}
$$

(for any $u \in U$ ) and

$$
j: e_{0} \mapsto e_{0}, e_{v} \mapsto \frac{1}{q^{n}+1} \sum_{0 \neq w \in U,\langle v, w\rangle=0} e_{w}-\frac{q^{n}+2}{q^{n}\left(q^{n}+1\right)} \sum_{w \in U,\langle w, v\rangle \neq 0} e_{w}
$$

(for any $0 \neq v \in U$ ). One can check that $D:=\langle\boldsymbol{\delta}, \boldsymbol{j}\rangle \simeq O_{2}^{+}(q)$ and that $D$ centralizes $S$. The subgroup $S \times D$ of $G L(E)$ is the desired dual pair $S p_{2 n}(q) \times O_{2}^{+}(q)$. Let $\omega_{n}$ denote the character of $S \times D$ acting on $E$. It is shown in [T] that $\left.\omega_{n}\right|_{S}=\tau_{n}$, and moreover one can label the irreducible characters of $D$ as $\beta=1_{D}, \alpha$ of degree 1 , and $\mu_{i}, 1 \leq i \leq(q-2) / 2$, of degree 2 such that

$$
\left.\omega_{n}\right|_{S \times D}=\left(\rho_{n}^{1}+1_{S}\right) \otimes \alpha+\left(\rho_{n}^{2}+1_{S}\right) \otimes \beta+\sum_{i=1}^{(q-2) / 2} \tau_{n}^{i} \otimes \mu_{i} .
$$

We can repeat the above construction but with $n$ replaced throughout by $n-1$, resp. by 1 , and subscript 1 , resp. 2, attached to all letters $U, E, S, D, \delta$, and $j$. Thus we get the dual pair $S_{1} \times D_{1} \simeq S p_{2 n-2}(q) \times O_{2}^{+}(q)$ inside $G L\left(E_{1}\right)$ with character $\omega_{n-1}$, and the dual pair $S_{2} \times D_{2} \simeq S p_{2}(q) \times O_{2}^{+}(q)$ inside $G L\left(E_{2}\right)$ with character $\omega_{1}$. Now we can identify $U$ with $U_{1} \oplus U_{2}$. This in turn identifies $E$ with $E_{1} \otimes E_{2}$ and $\boldsymbol{\delta}$ with $\boldsymbol{\delta}_{1} \otimes \boldsymbol{\delta}_{2}$. This identification also embeds $S_{1} \otimes S_{2}$ in $S$. In what follows, we denote $x_{1}:=\boldsymbol{\delta}_{1}^{a} \boldsymbol{j}_{1}^{b}$ and $x_{2}:=\boldsymbol{\delta}_{2}^{a} \boldsymbol{j}_{2}^{b}$ for $x=\boldsymbol{\delta}^{a} \boldsymbol{j}^{b}$.

Lemma 7.6. Let $S p_{2 n-2}(q) \times S p_{2}(q)$ be a standard subgroup of $S p_{2 n}(q)$. Then $\omega_{n}(g x)=\omega_{n-1}\left(h x_{1}\right) \cdot \omega_{1}\left(x_{2}\right)$ for any $x \in O_{2}^{+}(q)$ and any $g=h \otimes 1 \in S p_{2 n-2}(q) \times$ $S p_{2}(q)$.

Proof. Suppose first that $x=\boldsymbol{\delta}^{a}$. Then $g x=(h \otimes 1)\left(\boldsymbol{\delta}_{1} \otimes \boldsymbol{\delta}_{2}\right)^{a}=h \boldsymbol{\delta}_{1}^{a} \otimes \boldsymbol{\delta}_{2}^{a}=h x_{1} \otimes x_{2}$, whence the statement follows by taking trace.

Next assume that $x=\boldsymbol{\delta}^{a} \boldsymbol{j}$. Then $\omega_{1}\left(x_{2}\right)=q$. Let

$$
\begin{aligned}
N & :=\left|\left\{w \in U \mid w \neq 0,\left\langle\boldsymbol{\delta}^{-a} g^{-1}(w), w\right\rangle=0\right\}\right|, \\
N_{1} & :=\left|\left\{w \in U_{1} \mid w \neq 0,\left\langle\boldsymbol{\delta}_{1}^{-a} h^{-1}(w), w\right\rangle=0\right\}\right| .
\end{aligned}
$$

Then $\omega_{n}(g x)=2 q^{-n}(N+1)-q^{n}, \omega_{n-1}\left(h x_{1}\right)=2 q^{1-n}\left(N_{1}+1\right)-q^{n-1}$. Direct computation shows that $N+1=q^{2}\left(N_{1}+1\right)$, whence $\omega_{n}(g x)=q \omega_{n-1}\left(h x_{1}\right)$ as stated.

Proposition 7.7. Let $S=S p_{2 n}(q)$ with $n \geq 2$, and let $H=S p_{2 n-2}(q)$ be a standard subgroup of $S$. Then, for $1 \leq i \leq q / 2-1$,

$$
\begin{aligned}
& \left.\rho_{n}^{1}\right|_{H}=(q+1) \cdot 1_{H}+(q+1) \rho_{n-1}^{1}+\rho_{n-1}^{2}+(q+1) \sum_{k=1}^{(q-2) / 2} \tau_{n-1}^{k}, \\
& \left.\rho_{n}^{2}\right|_{H}=(q+1) \cdot 1_{H}+(q+1) \rho_{n-1}^{2}+\rho_{n-1}^{1}+(q+1) \sum_{k=1}^{(q-2) / 2} \tau_{n-1}^{k}, \\
& \left.\tau_{n}^{i}\right|_{H}=\tau_{n-1}^{i}+(q+1)\left(2 \cdot 1_{H}+2 \sum_{k=1}^{(q-2) / 2} \tau_{n-1}^{k}+\rho_{n-1}^{1}+\rho_{n-1}^{2}\right) .
\end{aligned}
$$


Proof. 1) The standard embedding of $H$ in $S$ can be written as $h \in H \mapsto g=h \otimes 1 \in$ $S$. In the notation of $\S 3$ it follows that $\operatorname{dim}_{\mathbb{F}_{q}} \operatorname{Ker}\left(g-\delta^{j}\right)$ equals $2+\operatorname{dim}_{\mathbb{F}_{q}} \operatorname{Ker}\left(h-\delta^{j}\right)$ if $j=0$ and $\operatorname{dim}_{\mathbb{F}_{q}} \operatorname{Ker}\left(h-\delta^{j}\right)$ if $j \neq 0$. Hence (1) implies that

$\left.\tau_{n}^{i}\right|_{H}=\tau_{n-1}^{i}+(q+1) \tau_{n-1}=\tau_{n-1}^{i}+(q+1)\left(2 \cdot 1_{H}+2 \sum_{k=1}^{(q-2) / 2} \tau_{n-1}^{k}+\rho_{n-1}^{1}+\rho_{n-1}^{2}\right)$

for any $i=1, \ldots, q-2$. Similarly,

$$
\left.\tau_{n}^{0}\right|_{H}=2(q+1) \cdot 1_{H}+(q+2)\left(\rho_{n-1}^{1}+\rho_{n-1}^{2}\right)+2(q+1) \sum_{k=1}^{(q-2) / 2} \tau_{n-1}^{k} .
$$

2) According to (19), $\rho_{n}^{2}+1_{S}$ is just the $S$-character of the $1_{D}$-homogeneous component of $\omega_{n}$, whence

$$
\rho_{n}^{2}(g)=\frac{1}{2(q-1)} \sum_{x \in D} \omega_{n}(g x)-1
$$

If $g=h \otimes 1$ as in 1), then $\omega_{n}(g x)=\omega_{n-1}(h x) \cdot \omega_{2}(x)$ by Lemma 7.6 Here we have identified $x_{1}$ and $x_{2}$ with no loss. Moreover, if $0 \leq a \leq q$ and $b=0,1$, then $\omega_{2}\left(\boldsymbol{\delta}^{a} \boldsymbol{j}^{b}\right)$ equals $q^{2}$, resp. $q$, or 1 , provided that $(a, b)=(0,0)$, resp. $b=1$, or $(a, b)=(\neq 0,0)$. It follows that

$$
\begin{aligned}
& \rho_{n}^{2}(g)=\left(q^{2} \omega_{n-1}(h)+\sum_{a=1}^{q-2} \omega_{n-1}\left(h \boldsymbol{\delta}^{a}\right)+q \sum_{a=0}^{q-2} \omega_{n-1}\left(h \boldsymbol{\delta}^{a} \boldsymbol{j}\right)\right) / 2(q-1)-1, \\
& \rho_{n-1}^{2}(h)=\left(\omega_{n-1}(h)+\sum_{a=1}^{q-2} \omega_{n-1}\left(h \boldsymbol{\delta}^{a}\right)+\sum_{a=0}^{q-2} \omega_{n-1}\left(h \boldsymbol{\delta}^{a} \boldsymbol{j}\right)\right) / 2(q-1)-1 .
\end{aligned}
$$

Therefore

$$
\begin{gathered}
\rho_{n}^{2}(g)-q \rho_{n-1}^{2}(h)=\frac{1}{2}\left(q \omega_{n-1}(h)-\sum_{a=1}^{q-2} \omega_{n-1}\left(h \boldsymbol{\delta}^{a}\right)\right)+(q-1) \\
=\frac{1}{2}\left((q+1) \omega_{n-1}(h)-\left(\sum_{a=0}^{q-2} \omega_{n-1}\left(\boldsymbol{\delta}^{a} h\right)-2\right)\right)=\frac{q+1}{2} \cdot \omega_{n-1}(h)-\frac{q-1}{2} \cdot \tau_{n-1}^{0}(h) \\
=\frac{q+1}{2}\left(2 \cdot 1_{H}+\rho_{n-1}^{1}+\rho_{n-1}^{2}+2 \sum_{k=1}^{(q-2) / 2} \tau_{n-1}^{k}\right)(h)-\frac{q-1}{2}\left(\rho_{n-1}^{1}+\rho_{n-1}^{2}\right)(h) \\
=(q+1) \cdot 1_{H}+\rho_{n-1}^{1}+\rho_{n-1}^{2}+(q+1) \sum_{k=1}^{(q-2) / 2} \tau_{n-1}^{k}(h) .
\end{gathered}
$$

Thus we have proved the branching formula for $\rho_{n}^{2}$, and so we are also done for $\rho_{n}^{1}$ because of (20). 
Corollary 7.8. Let $G=S p_{2 n}(q)$ with $n \geq 2$ and let $1 \leq i, j \leq q / 2-1$. Then

$$
\begin{gathered}
\rho_{n}^{1}(\boldsymbol{t})=\rho_{n}^{2}(\boldsymbol{t})=\frac{q^{2 n-1}-q}{2(q-1)}, \tau_{n}^{i}(\boldsymbol{t})=\frac{q^{2 n-1}-1}{q-1}, \\
\rho_{n}^{1}(\boldsymbol{s})=\frac{q^{2 n-2}-q^{n+1}+q^{n}-q}{2(q-1)}, \rho_{n}^{2}(\boldsymbol{s})=\frac{q^{2 n-2}+q^{n+1}-q^{n}-q}{2(q-1)}, \tau_{n}^{i}(\boldsymbol{s})=\frac{q^{2 n-2}-1}{q-1}, \\
\rho_{n}^{1}(\boldsymbol{d})=\rho_{n}^{2}(\boldsymbol{d})=\frac{q^{2 n-2}-q}{2(q-1)}, \tau_{n}^{i}(\boldsymbol{d})=\frac{q^{2 n-2}-1}{q-1}, \\
\rho_{n}^{1}\left(\boldsymbol{c}_{j}\right)=\frac{\left(q^{n-1}+1\right)\left(q^{n-1}-q\right)}{2(q-1)}+1, \rho_{n}^{2}\left(\boldsymbol{c}_{j}\right)=\frac{\left(q^{n-1}-1\right)\left(q^{n-1}+q\right)}{2(q-1)}+1, \\
\tau_{n}^{i}\left(\boldsymbol{c}_{j}\right)=\frac{q^{2 n-2}-1}{q-1}+\tilde{\delta}^{i j}+\tilde{\delta}^{-i j}
\end{gathered}
$$

Proof. Argue as in the proof of Corollary 7.3 using Proposition 7.7 .

For any linear $Q_{1}$-character $\lambda$, let $\hat{\lambda}$ be the character of $K_{\lambda}=Q_{1}: I_{\lambda}$, where $\left.\hat{\lambda}\right|_{Q_{1}}=\lambda$ and $\left.\hat{\lambda}\right|_{I_{\lambda}}=1_{I_{\lambda}}$. In what follows, the $P_{1}$-module $\boldsymbol{A}_{\varepsilon}$ is defined to be $\operatorname{Ind}_{K_{\lambda}}^{P_{1}}(\hat{\lambda})$ for $\lambda \in \mathcal{O}_{2}^{\varepsilon}$, and the $P_{1}$-module $\boldsymbol{C}$ is defined to be $\operatorname{Ind}_{K_{\lambda}}^{P_{1}}(\hat{\lambda})$ for $\lambda \in \mathcal{O}_{1}$. Let $\delta_{j}$ be the linear character of $P_{1} / P_{1}^{\prime} \simeq T_{1}=\left\langle c_{1}\right\rangle$ that sends $c_{1}$ to $\tilde{\delta}^{j}$. In the next statement, we will consider $\rho_{n-1}^{1} \otimes 1_{T_{1}}$ as a character of $L_{1}=L_{1}^{\prime} \times T_{1}$, and then inflate it to $P_{1}=Q_{1} L_{1}$. Similarly for $\rho_{n-1}^{2} \otimes 1_{T_{1}}$ and $\tau_{n-1}^{i} \otimes 1_{T_{1}}$. We will also consider $\delta_{j}$ as a character of $P_{1}$.

Proposition 7.9. Let $G=S p_{2 n}(q)$ with $n \geq 3,(n, q) \neq(3,2),(4,2)$, and let $1 \leq i \leq q / 2-1$. Then

$$
\begin{gathered}
\left.\rho_{n}^{1}\right|_{P_{1}}=1_{P_{1}}+\rho_{n-1}^{1} \otimes 1_{T_{1}}+\boldsymbol{C}+\boldsymbol{A}_{-},\left.\quad \rho_{n}^{2}\right|_{P_{1}}=1_{P_{1}}+\rho_{n-1}^{2} \otimes 1_{T_{1}}+\boldsymbol{C}+\boldsymbol{A}_{+}, \\
\left.\tau_{n}^{i}\right|_{P_{1}}=\delta_{i}+\delta_{-i}+\tau_{n-1}^{i} \otimes 1_{T_{1}}+\boldsymbol{C}\left(\delta_{i}+\delta_{-i}\right)+\boldsymbol{A}_{+}+\boldsymbol{A}_{-} .
\end{gathered}
$$

Proof. Define the following $Q_{1}$-characters:

$$
\omega_{1}:=\sum_{\lambda \in \mathcal{O}_{1}} \lambda, \omega_{2}^{+}:=\sum_{\lambda \in \mathcal{O}_{2}^{+}} \lambda, \omega_{2}^{-}:=\sum_{\lambda \in \mathcal{O}_{2}^{-}} \lambda .
$$

1) Here we show that $\left.\tau_{n}^{0}\right|_{P_{1}}$ contains $2 \boldsymbol{C}$; in particular, $\left(\rho_{n}^{1}+\rho_{n}^{2}\right)_{Q_{1}}$ contains $2 \omega_{1}$. Recall that $Q_{1}=\left\{[A, C] \mid A \in \mathbb{F}_{q}^{2 n-2}, C \in \mathbb{F}_{q}\right\}$ (with respect to the basis $\left.\left(e_{1}, \ldots, e_{n}, f_{2}, \ldots, f_{n}, f_{1}\right)\right)$, and we can write any character $\lambda \in \mathcal{O}_{1}$ in the form $\lambda=\lambda_{B}:[A, C] \mapsto(-1)^{\operatorname{tr}_{\mathbb{F}_{q} / \mathbb{F}_{2}}\left({ }^{t} b J_{n-1} A\right)}$ for some $0 \neq b \in \mathbb{F}_{q}^{2 n-2}$. For such $\lambda=\lambda_{B}$ we have $K_{\lambda}:=\operatorname{Stab}_{P_{1}}(\lambda)=Q_{1}: I_{\lambda}$, where

$$
I_{\lambda}=\left\{\operatorname{diag}\left(x, h, x^{-1}\right) \mid x \in \mathbb{F}_{q}^{\bullet}, h \in L_{1}^{\prime}, h(b)=x b\right\} .
$$

Identifying $\mathbb{F}_{q}^{2 n-2}$ with $\left\langle e_{2}, \ldots, f_{n}, f_{2}, \ldots, f_{n}\right\rangle_{\mathbb{F}_{q}}$ and choosing $b$ to be $e_{2}$, we see that

$$
K_{\lambda}=\left\{g \in G \mid g\left(e_{1}\right)=x e_{1}, g\left(e_{2}\right) \equiv x e_{2}\left(\bmod \left\langle e_{1}\right\rangle_{\mathbb{F}_{q}}\right), x \in \mathbb{F}_{q}^{\bullet}\right\} .
$$

By Mackey's formula and Frobenius' reciprocity,

$$
\left(\left.\operatorname{Ind}_{P_{1}}^{G}\left(1_{P_{1}}\right)\right|_{P_{1}}, \boldsymbol{C}\right)_{P_{1}}=\left.\left(1_{P_{1}},\left.\operatorname{Ind}_{P_{1}}^{G}(\boldsymbol{C})\right|_{P_{1}}\right)\right|_{P_{1}}=\sum_{g \in P_{1} \backslash G / K_{\lambda}}\left(1_{P_{1}^{g} \cap K_{\lambda}},\left.\hat{\lambda}\right|_{P_{1}^{g} \cap K_{\lambda}}\right)_{P_{1}^{g} \cap K_{\lambda}} .
$$

Choose $g_{1}, g_{2} \in G$ such that $g_{1}\left(e_{1}\right)=f_{1}$ and $g_{2}\left(e_{1}\right)=e_{2}$, and consider the following two conjugates $P_{11}:=P_{1}^{g_{1}}, P_{12}:=P_{1}^{g_{2}}$ of $P_{1}$. Observe that the double cosets 
$P_{1} g_{1} K_{\lambda}$ and $P_{1} g_{2} K_{\lambda}$ are distinct. One can also check that $K_{\lambda} \cap P_{11}=I_{\lambda} \leq \operatorname{Ker}(\hat{\lambda})$ and $K_{\lambda} \cap P_{12} \leq \operatorname{Ker}(\hat{\lambda})$. It follows that $\left(\left.\operatorname{Ind}_{P_{1}}^{G}\left(1_{P_{1}}\right)\right|_{P_{1}}, C\right)_{P_{1}} \geq 2$, as stated.

2) Notice that $\tau_{n}^{i}=\operatorname{Ind}_{P_{1}}^{G}\left(\delta_{i}\right)$. So by Mackey's formula and Frobenius' reciprocity, for any $j$ we have

$$
\begin{aligned}
\left(\left.\tau_{n}^{i}\right|_{P_{1}}, \boldsymbol{C} \delta_{j}\right)_{P_{1}} & =\left(\left.\operatorname{Ind}_{P_{1}}^{G}\left(\delta_{i}\right)\right|_{P_{1}}, \operatorname{Ind}_{K_{\lambda}}^{P_{1}}\left(\hat{\lambda} \delta_{i}\right)\right)_{P_{1}} \\
& =\sum_{g \in P_{1} \backslash G / K_{\lambda}}\left(\left.\delta_{i}^{g}\right|_{P_{1}^{g} \cap K_{\lambda}},\left.\hat{\lambda} \delta_{j}\right|_{P_{1}^{g} \cap K_{\lambda}}\right)_{P_{1}^{g} \cap K_{\lambda}} .
\end{aligned}
$$

Consider the elements $g_{1}$ and $g_{2}$ as in 1). For $j=i$ observe that $\delta_{i}^{g_{2}}$ and $\hat{\lambda} \delta_{i}$ agree on $P_{12}:=P_{1}^{g_{2}}$. For $j=-i$ observe that $\delta_{i}^{g_{1}}$ and $\hat{\lambda} \delta_{-i}$ agree on $P_{11}:=P_{1}^{g_{1}}$. It follows that $\left.\tau_{n}^{i}\right|_{P_{1}}$ contains both $\boldsymbol{C} \delta_{i}$ and $\boldsymbol{C} \delta_{-i}$. Also notice that the last two characters are distinct, as they take different values at $\operatorname{diag}\left(\delta, \delta, I_{n-2}, \delta^{-1}, I_{n-2}, \delta^{-1}\right)$.

3) Observe that $\rho_{n}^{1}(1)<\rho_{n}^{2}(1)<2 \cdot\left|\mathcal{O}_{1}\right|+\left|\mathcal{O}_{2}^{-}\right|$and $\left|\mathcal{O}_{2}^{-}\right|<\left|\mathcal{O}_{2}^{+}\right|$. On the other hand, $\left.\rho_{n}^{1}\right|_{Q_{1}}$ contains either $\omega_{2}^{-}$or $\omega_{2}^{+}$; similarly for $\left.\rho_{n}^{2}\right|_{Q_{1}}$. It follows that neither $\left.\rho_{n}^{1}\right|_{Q_{1}}$ nor $\left.\rho_{n}^{2}\right|_{Q_{1}}$ can contain $2 \omega_{1}$. Together with 1$)$, this implies that the multiplicity of $\boldsymbol{C}$ in $\left.\rho_{n}^{1}\right|_{P_{1}}$ and $\left.\rho_{n}^{2}\right|_{P_{1}}$ is exactly 1 (since $\left.\boldsymbol{C}\right|_{Q_{1}}=\omega_{1}$ ). Also by Frobenius' reciprocity, the multiplicity of $1_{P_{1}}$ in $\left.\rho_{n}^{1}\right|_{P_{1}}$ and $\left.\rho_{n}^{2}\right|_{P_{1}}$ is exactly 1 .

4) Let $V$ be a module affording the character $\rho_{n}^{1}$, and write $V=C_{V}\left(Z_{1}\right) \oplus\left[Z_{1}, V\right]$. The character of the $P_{1}$-module $\left[Z_{1}, V\right]$ can be written as $\phi_{+}+\phi_{-}$, where $\phi_{+} \mid Q_{Q_{1}}=$ $a \omega_{2}^{+}$and $\left.\phi_{-}\right|_{Q_{1}}=b \omega_{2}^{-}$for $0 \leq a, b \in \mathbb{Z}$. For any hyperplane $H$ of type $\varepsilon$ of $Q_{1}$ there are $(q-1)$ characters $\lambda \in \mathcal{O}_{2}^{\varepsilon}$ that are trivial on $H$, and their restrictions to $Z_{1} \simeq Q_{1} / H$ exhaust $\operatorname{Irr}\left(Z_{1}\right) \backslash\left\{1_{Z_{1}}\right\}$. It follows that

$\phi_{+}(\boldsymbol{t})=-\frac{\phi_{+}(1)}{q-1}=-a q^{n-1}\left(q^{n-1}+1\right) / 2, \phi_{-}(\boldsymbol{t})=-\frac{\phi_{-}(1)}{q-1}=-b q^{n-1}\left(q^{n-1}-1\right) / 2$

for any transvection $\boldsymbol{t} \in Z_{1}$. Thus

$$
\begin{gathered}
\rho_{n}^{1}(1)=\operatorname{dim}\left(C_{V}\left(Z_{1}\right)\right)+a q^{n-1}\left(q^{n-1}+1\right)(q-1) / 2+b q^{n-1}\left(q^{n-1}-1\right)(q-1) / 2 \\
\left(q^{2 n-1}-q\right) / 2(q-1)=\rho_{n}^{1}(\boldsymbol{t}) \\
=\operatorname{dim}\left(C_{V}\left(Z_{1}\right)\right)-a q^{n-1}\left(q^{n-1}+1\right) / 2-b q^{n-1}\left(q^{n-1}-1\right) / 2 .
\end{gathered}
$$

Therefore $a\left(q^{n-1}+1\right)+b\left(q^{n-1}-1\right)=q^{n-1}-1$, i.e. $(a, b)=(0,1)$. Also, $\operatorname{dim}\left(C_{V}\left(Z_{1}\right)\right)=1+\rho_{n-1}^{1}(1)+\left|\mathcal{O}_{1}\right|$. Since the $Q_{1}$-module $C_{V}\left(Z_{1}\right)$ contains the character $\omega_{1}$ by 3$)$, Proposition 7.7 now implies that the $P_{1}^{\prime}$-module $C_{V}\left(Z_{1}\right)$ affords the character $1+\rho_{n-1}^{1}+\left.\boldsymbol{C}\right|_{P_{1}^{\prime}}$. By Corollary 4.2, Proposition 5.7, and Theorem 5.11, for any $\lambda \in \mathcal{O}_{2}^{-}, K_{\lambda}$ acts on the $\lambda$-homogeneous component via $\hat{\lambda}$, whence $\phi_{-}=\boldsymbol{A}_{-}$. We have shown that

$$
\left.\rho_{n}^{1}\right|_{P_{1}}=1_{P_{1}}+\rho_{n-1}^{1} \otimes \alpha+\boldsymbol{C}+\boldsymbol{A}_{-}
$$

for some linear character $\alpha$ of $T_{1}$.

In the same fashion we can show that

$$
\left.\rho_{n}^{2}\right|_{P_{1}}=1_{P_{1}}+\rho_{n-1}^{2} \otimes \beta+\boldsymbol{C}+\boldsymbol{A}_{+}
$$

for some linear character $\beta$ of $T_{1}$.

5) From 4) it follows that $\left.\tau_{n}^{0}\right|_{Q_{1}}=\left(2+\tau_{n-1}^{0}(1)\right) \cdot 1_{Q_{1}}+2 \omega_{1}+\omega_{2}^{+}+\omega_{2}^{-}$. By Lemma $3.8 \tau_{n}^{i}(g)-\tau_{n}^{0}(g)=1$ for any $g \in Q_{1}$. Hence $\left.\tau_{n}^{i}\right|_{Q_{1}}=\left(2+\tau_{n-1}^{i}(1)\right) \cdot 1_{Q}+2 \omega_{1}+\omega_{2}^{+}+\omega_{2}^{-}$. By Corollary 4.2, Proposition 5.7, and Theorem 5.11, $\omega_{2}^{+}$lifts to the $P_{1}$-character $\boldsymbol{A}_{+}$, and $\omega_{2}^{-}$lifts to the $P_{1}$-character $\boldsymbol{A}_{-}$. Since $\left.\boldsymbol{C} \delta_{ \pm i}\right|_{Q_{1}}=\omega_{1}, 2 \omega_{1}$ lifts to the 
$P_{1}$-character $\boldsymbol{C}\left(\delta_{i}+\delta_{-i}\right)$ by the result of 2). Clearly, $\tau_{n}^{q-1-i}=\tau_{n}^{i}=\operatorname{Ind}_{P_{1}}^{G}\left(\delta_{i}\right)$, whence $\left.\tau_{n}^{i}\right|_{P_{1}}$ contains $\delta_{i}$ and $\delta_{-i}$ by Frobenius' reciprocity. It also follows from 4) that

$$
\left.\left(\boldsymbol{C}\left(\delta_{i}+\delta_{-i}\right)+\boldsymbol{A}_{-}+\boldsymbol{A}_{+}\right)\right|_{L_{1}^{\prime}}=2 q \cdot 1_{L_{1}^{\prime}}+(q+1)\left(2 \sum_{k=1}^{(q-2) / 2} \tau_{n-1}^{k}+\rho_{n-1}^{1}+\rho_{n-1}^{2}\right) .
$$

Together with Proposition 7.7 this implies that the constituent $\left(2+\tau_{n-1}^{i}(1)\right) \cdot 1_{Q_{1}}$ of $\left.\tau_{n}^{i}\right|_{Q_{1}}$ yields the $L_{1}^{\prime}$-character $2 \cdot 1_{L_{1}^{\prime}}+\tau_{n-1}^{i}$. We have shown that

$$
\left.\tau_{n}^{i}\right|_{P_{1}}=\delta_{i}+\delta_{-i}+\tau_{n-1}^{i} \otimes \gamma+\boldsymbol{C}\left(\delta_{i}+\delta_{-i}\right)+\boldsymbol{A}_{+}+\boldsymbol{A}_{-}
$$

for some linear character $\gamma$ of $T_{1}$.

6 ) It remains to identify the $T_{1}$-characters $\alpha, \beta$, and $\gamma$. Consider the semisimple elements $\boldsymbol{c}_{j}$ with $1 \leq j \leq q / 2-1$. Since $\boldsymbol{c}_{j} \in L_{1} \backslash L_{1}^{\prime}$ and $\operatorname{Stab}_{L_{1}}(\lambda)<L_{1}^{\prime}$ for any $\lambda \in \mathcal{O}_{2}^{\varepsilon}, \boldsymbol{A}_{+}\left(\boldsymbol{c}_{j}\right)=\boldsymbol{A}_{-}\left(\boldsymbol{c}_{j}\right)=0$. Also, $\boldsymbol{c}_{j}$ fixes no $\lambda \in \mathcal{O}_{1}$, whence $\left(\boldsymbol{C} \delta_{k}\right)\left(\boldsymbol{c}_{j}\right)=0$ for any $k$. Thus the result of 5) implies that $\tau_{n}^{i}\left(\boldsymbol{c}_{j}\right)=\tilde{\delta}^{i j}+\tilde{\delta}^{-i j}+\tau_{n-1}^{i}(1) \cdot \gamma\left(\boldsymbol{c}_{j}\right)$. But $\tau_{n}^{i}\left(\boldsymbol{c}_{j}\right)=\tilde{\delta}^{i j}+\tilde{\delta}^{-i j}+\tau_{n-1}^{i}(1)$ by Corollary [7.8. Consequently, $\gamma\left(\boldsymbol{c}_{j}\right)=1$ for any $j$, i.e. $\gamma=1_{T_{1}}$. In the same fashion one can show that $\alpha=\beta=1_{T_{1}}$.

Corollary 7.10. Let $G=S p_{2 n}(q)$ with $n \geq 2$. Write $q-1=\ell^{a} r$ with $(r, \ell)=1$.

(i) Let $\varphi$ be any nontrivial composition factor of $\hat{\rho}_{n}^{1}$, $\hat{\rho}_{n}^{2}$, or $\hat{\tau}_{n}^{i}$. Then $\varphi$ is of form $\rho-a \cdot 1_{G}$, where $\rho \in\left\{\hat{\rho}_{n}^{1}, \hat{\rho}_{n}^{2}, \hat{\tau}_{n}^{j} \mid 1 \leq j \leq(r-1) / 2\right\}$ and $a=0$ or 1 . Moreover, $a=1$ exactly when either $\ell \mid\left(q^{n}-1\right) /(q-1)$ and $\rho=\hat{\rho}_{n}^{1}$, or $\ell \mid\left(q^{n}+1\right)$ and $\rho=\hat{\rho}_{n}^{2}$.

(ii) The Brauer characters $\hat{\rho}_{n}^{1}, \hat{\rho}_{n}^{2}, \hat{\tau}_{n}^{j}$ with $1 \leq j \leq(r-1) / 2$, each have a unique nontrivial irreducible constituent, and these $(r+3) / 2$ constituents are pairwise distinct. This statement also holds for $n=1$ if we remove $\hat{\rho}_{1}^{1}$ from this list.

Proof. (i) The statement for $n=2$, resp. for $(n, q)=(3,2)$ and $(4,2)$, can be checked directly using Wh1, resp. JLPW. We will proceed by induction on $n$ and assume $n \geq 3$ and $(n, q) \neq(3,2),(4,2)$.

1) Arguing as in the proof of [DT, Theorem 7.2] one can show that

$$
\hat{\tau}_{n}^{i}-\hat{\tau}_{n}^{j}=-\delta_{i, 0}+\delta_{j, 0},
$$

whenever $0 \leq i, j \leq q-2$ and $i \equiv j(\bmod r)$. Together with (2) and (3), it implies that any nontrivial composition factor of $\hat{\tau}_{n}^{i}$ is a composition factor of $\hat{\rho}_{n}^{1}, \hat{\rho}_{n}^{2}$, or $\hat{\tau}_{n}^{j}$ with $1 \leq j \leq(r-1) / 2$.

2) Consider the semisimple character $\psi_{j}$ labelled by $s_{j} \in G$ conjugate to $\operatorname{diag}\left(\delta^{j}, \delta^{-j}, I_{2 n-2}\right)$ with $1 \leq j \leq(r-1) / 2$. Since the $\ell^{\prime}$-part $s_{j}^{\prime}$ of $s_{j}$ is nontrivial semisimple and $\psi_{j}(1)=\left(q^{2 n}-1\right) /(q-1)=\left(G: C_{G}\left(s_{j}^{\prime}\right)\right)_{2^{\prime}}$, [HM Proposition 1] implies that $\widehat{\psi}_{j}$ is irreducible. Moreover, these $(r-1) / 2$ characters $\psi_{j}$ belong to $(r-1) / 2$ distinct nonunipotent $\ell$-blocks, since the $s_{j}^{\prime}$ are pairwise nonconjugate and nontrivial. On the other hand, by Corollary 6.3, $\left\{\psi_{j} \mid 1 \leq j \leq(r-1) / 2\right\} \subseteq\left\{\tau_{n}^{i} \mid\right.$ $1 \leq i \leq q / 2-1\}$. The result of 1$)$ shows that there are at most $(r-1) / 2$ distinct irreducible Brauer characters of degree $\left(q^{2 n}-1\right) /(q-1)$ that can arise as composition factors of $\hat{\tau}_{n}^{i}$. We conclude that the Brauer characters $\hat{\tau}_{n}^{j}$ with $1 \leq j \leq(r-1) / 2$ are irreducible and pairwise distinct.

3) Notice that $1_{G}+\rho_{n}^{1}+\rho_{n}^{2}$ is the rank 3 permutation character for $G$, whence the statement that $\rho-a \cdot 1_{G} \in \operatorname{IBr}_{\ell}(G)$ for a suitable $a=0,1$ when $\rho \in\left\{\hat{\rho}_{n}^{1}, \hat{\rho}_{n}^{2}\right\}$ follows from $\left[\mathrm{Li}\right.$. Furthermore, it is shown in $[\mathrm{ST}]$ that $\hat{\rho}_{n}^{1}$ is reducible if and only 
if $\ell \mid\left(q^{n}-1\right) /(q-1)$, and $\hat{\rho}_{n}^{2}$ is reducible if and only if $\ell \mid\left(q^{n}+1\right)$. Thus (i) has been established.

(ii) follows from (i) if $n \geq 2$. The statement for $n=1$ is obvious.

In view of Corollaries 7.5] and [7.10, define

$$
\mathfrak{W}_{+}:=\left\{1_{G}, \hat{\rho}_{n}^{1}, \hat{\rho}_{n}^{2}, \hat{\tau}_{n}^{j} \mid 1 \leq j \leq(r-1) / 2\right\},
$$

where $r=(q-1)_{\ell^{\prime}}$, and

$$
\mathfrak{W}_{-}:=\left\{1_{G}, \widehat{\alpha}_{n}, \widehat{\beta}_{n}, \hat{\zeta}_{n}^{j} \mid 1 \leq j \leq\left(r^{\prime}-1\right) / 2\right\},
$$

where $r^{\prime}=(q+1)_{\ell^{\prime}}$. By Corollary 7.10, any linear-Weil (Brauer) character is a $\mathbb{Z}$ combination of characters from $\mathfrak{W}_{+}$. Similarly, any unitary-Weil (Brauer) character is a $\mathbb{Z}$-combination of characters from $\mathfrak{W}_{-}$by Corollary 7.5 .

Lemma 7.11. Let $G=S p_{2 n}(q)$.

(i) Let $n \geq 1$ and let $\varphi$ be a $\mathbb{Z}$-combination of trivial and linear-Weil Brauer characters. Suppose that $\left.\varphi\right|_{P_{1}}=0$. Then $\varphi=0$.

(ii) Let $n \geq 2$ and let $\varphi$ be a $\mathbb{Z}$-combination of trivial and unitary-Weil Brauer characters. Suppose that $\left.\varphi\right|_{P_{1}}=0$. Then $\varphi=0$.

Proof. (i) Write $(q-1)=\ell^{k} r$ with $(r, \ell)=1$. Then $\varphi=a \hat{\rho}_{n}^{1}+b \hat{\rho}_{n}^{2}+\sum_{i=1}^{(r-1) / 2} c_{i} \hat{\tau}_{n}^{i}+$ $d \cdot 1_{G}$ for some $a, b, c_{i}, d \in \mathbb{Z}$. The case $n=1$ can be checked directly using character table, so we assume that $n \geq 2$. By assumption, $\varphi(g)=0$ for $g=1, \boldsymbol{t}, \boldsymbol{s}, \boldsymbol{d}$, and $\boldsymbol{c}_{0}^{j}$ with $1 \leq j \leq(r-1) / 2$, where $\boldsymbol{c}_{0}:=\boldsymbol{c}_{\ell^{k}}$. The conditions $\varphi(1)=\varphi(\boldsymbol{t})=\varphi(\boldsymbol{s})=$ $\varphi(\boldsymbol{d})=0$ imply by Corollary 7.8 that $a=b=d=0$ and

$$
\sum_{i=1}^{(r-1) / 2} c_{i}=0
$$

Let $\tilde{\delta}_{0}:=\delta^{\ell^{k}}$. By Corollary 7.8 and (21),

$$
0=\varphi\left(\boldsymbol{c}_{0}^{j}\right)=\sum_{i=1}^{(r-1) / 2} c_{i}\left(\frac{q^{2 n-2}-1}{q-1}+\tilde{\delta}_{0}^{i j}+\tilde{\delta}_{0}^{-i j}\right)=\sum_{i=1}^{(r-1) / 2} c_{i}\left(\tilde{\delta}_{0}^{i j}+\tilde{\delta}_{0}^{-i j}\right) .
$$

Let $f(x):=\sum_{i=1}^{(r-1) / 2} c_{i}\left(x^{i}+x^{r-i}\right) \in \mathbb{Z}[x]$. It follows from (21) and (22) that $f\left(\tilde{\delta}_{0}^{j}\right)=0$ for $j=0,1, \ldots, r-1$. But $\operatorname{deg}(f) \leq r-1$, so $f \equiv 0$, i.e. $c_{i}=0$ for all $i$.

(ii) Write $(q+1)=\ell^{k} r$ with $(r, \ell)=1$. Then $\varphi=a \widehat{\alpha}_{n}+b \widehat{\beta}_{n}+\sum_{i=1}^{(r-1) / 2} c_{i} \hat{\zeta}_{n}^{i}+d \cdot 1_{G}$ for some $a, b, c_{i}, d \in \mathbb{Z}$. The case $n=2$, resp. $(n, q)=(3,2)$ or $(4,2)$, can be checked directly using the character table of $G$ [E], resp. [JLPW], so we assume that $n \geq 3$ and $(n, q) \neq(3,2),(4,2)$. By Proposition 7.4, the $Q_{1}$-fixed point part of $\varphi$ yields the (virtual) $L_{1}^{\prime}$-character

$$
\psi:=d \cdot 1_{L_{1}^{\prime}}+a \widehat{\alpha}_{n-1}+b \widehat{\beta}_{n-1}+\sum_{i=1}^{(r-1) / 2} c_{i} \hat{\zeta}_{n-1}^{i} .
$$

By our assumption, $\psi=0$. This implies by Corollary [7.5)(ii) that $a=b=c_{i}=0$ and $d=0$. 


\section{Restrictions to $P_{1}$ And $H_{d}$}

In this section we will show that any $\mathbb{F} G$-module with property $\left(\mathcal{W}_{2}^{\varepsilon}\right)$ agrees with a formal sum of Weil modules when restricted to $P_{1}$ and $H_{d}$.

Proposition 8.1. Let $G=S p_{2 n}(q)$ with $n \geq 3,(n, q) \neq(3,2),(4,2)$, and let $V$ be an irreducible $\mathbb{F} G$-module. Assume that $V$ satisfies $\left(\mathcal{W}_{2}^{-}\right)$. Then there is a formal sum $W$ of trivial and (irreducible) unitary-Weil modules of $G$ such that $\left.V\right|_{P_{1}}=\left.W\right|_{P_{1}}$.

Proof. Write $q+1=\ell^{k} r$ with $(r, \ell)=1$. Let $\varphi$ be the Brauer character of $V$.

1) Without loss we may assume that $V$ is nontrivial. By Proposition 5.7 and Theorem [5.11, $V$ satisfies the conclusion of Theorem 5.11(i). It follows that

$$
\left.V\right|_{P_{1}}=C_{V}\left(Q_{1}\right) \oplus n_{+} \widehat{\boldsymbol{B}}_{+} \oplus n_{-} \widehat{\boldsymbol{B}}_{-}
$$

for some nonnegative integers $n_{+}, n_{-}$(in the notation of \$7). According to (16) and (17), all nontrivial composition factors of $\left.\widehat{\boldsymbol{B}}_{+}\right|_{L_{1}^{\prime}}$ and $\left.\widehat{\boldsymbol{B}}_{-}\right|_{L_{1}^{\prime}}$ are unitary-Weil modules of $L_{1}^{\prime}$. Hence the same holds for $\left.C_{V}\left(Q_{1}\right)\right|_{L_{1}^{\prime}}$ by Lemma 2.1 By Corollary 7.5 applied to $L_{1}^{\prime}$,

$$
\left.C_{V}\left(Q_{1}\right)\right|_{L_{1}^{\prime}}=a \widehat{\alpha}_{n-1}+b \widehat{\beta}_{n-1}+\sum_{i=1}^{(r-1) / 2} c_{i} \hat{\zeta}_{n-1}^{i}+d \cdot 1_{L_{1}^{\prime}}
$$

for some $a, b, c_{i}, d \in \mathbb{Z}$. Notice that $a, b, c_{i} \geq 0$.

2) Here we compute $\varphi(\boldsymbol{t})$ and $\varphi\left(\boldsymbol{t}^{\prime}\right)$ for transvections $\boldsymbol{t} \in Z_{1}$ and $\boldsymbol{t}^{\prime} \in L_{1}^{\prime}$. By Proposition 7.4 and Corollary 7.3

$$
\begin{gathered}
\boldsymbol{B}_{-}(\boldsymbol{t})=\alpha_{n}(\boldsymbol{t})-\alpha_{n-1}(1)=\frac{-q^{2 n-2}+q^{n-1}}{2}, \\
\boldsymbol{B}_{-}\left(\boldsymbol{t}^{\prime}\right)=\alpha_{n}\left(\boldsymbol{t}^{\prime}\right)-\alpha_{n-1}\left(\boldsymbol{t}^{\prime}\right)=\frac{-q^{2 n-2}+q^{2 n-3}}{2} .
\end{gathered}
$$

Similarly,

$$
\boldsymbol{B}_{+}(\boldsymbol{t})=\left(-q^{2 n-2}-q^{n-1}\right) / 2, \boldsymbol{B}_{+}\left(\boldsymbol{t}^{\prime}\right)=\left(-q^{2 n-2}+q^{2 n-3}\right) / 2 .
$$

Now we have

$$
\begin{aligned}
\varphi(\boldsymbol{t})= & a \frac{\left(q^{n-1}-1\right)\left(q^{n-1}-q\right)}{2(q+1)}+b \frac{\left(q^{n-1}+1\right)\left(q^{n-1}+q\right)}{2(q+1)}+\sum_{i} c_{i} \frac{q^{2 n-2}-1}{q+1}+d \\
& +n_{+} \frac{-q^{2 n-2}-q^{n-1}}{2}+n_{-} \frac{-q^{2 n-2}+q^{n-1}}{2} \\
\varphi\left(\boldsymbol{t}^{\prime}\right)= & (a+b) \frac{-q^{2 n-3}+q}{2(q+1)}+\sum_{i} c_{i} \frac{-q^{2 n-3}-1}{q+1}+d+\left(n_{+}+n_{-}\right) \frac{-q^{2 n-2}+q^{2 n-3}}{2} .
\end{aligned}
$$

But $\varphi(\boldsymbol{t})=\varphi\left(\boldsymbol{t}^{\prime}\right)$, hence

$$
\left(a+\sum_{i} c_{i}-n_{-}\right)\left(q^{n-2}-1\right)+\left(b+\sum_{i} c_{i}-n_{+}\right)\left(q^{n-2}+1\right)=0 .
$$

3) Here we compute $\varphi(\boldsymbol{d})$ and $\varphi\left(\boldsymbol{d}^{\prime}\right)$ for double transvections $\boldsymbol{d}=\boldsymbol{t} \boldsymbol{t}^{\prime}$ (with $\boldsymbol{t} \in Z_{1}$ and $\boldsymbol{t}^{\prime} \in L_{1}^{\prime}$ as in 2)) and $\boldsymbol{d}^{\prime} \in L_{1}^{\prime}$. By Proposition 7.4 and Corollary 7.3 .

$$
\boldsymbol{B}_{-}(\boldsymbol{d})=\alpha_{n}(\boldsymbol{d})-\alpha_{n-1}\left(\boldsymbol{t}^{\prime}\right)=\frac{q^{2 n-3}}{2}, \boldsymbol{B}_{-}\left(\boldsymbol{d}^{\prime}\right)=\alpha_{n}\left(\boldsymbol{d}^{\prime}\right)-\alpha_{n-1}\left(\boldsymbol{d}^{\prime}\right)=\frac{q^{2 n-3}-q^{2 n-4}}{2} .
$$


Similarly,

$$
\boldsymbol{B}_{+}(\boldsymbol{t})=q^{2 n-3} / 2, \boldsymbol{B}_{+}\left(\boldsymbol{d}^{\prime}\right)=\left(q^{2 n-3}-q^{2 n-4}\right) / 2 .
$$

Now we have

$$
\begin{gathered}
\varphi(\boldsymbol{d})=(a+b) \frac{-q^{2 n-3}+q}{2(q+1)}+\sum_{i} c_{i} \frac{-q^{2 n-3}-1}{q+1}+d+\left(n_{+}+n_{-}\right) q^{2 n-3} / 2, \\
\varphi\left(\boldsymbol{d}^{\prime}\right)=(a+b) \frac{q^{2 n-4}+q}{2(q+1)}+\sum_{i} c_{i} \frac{q^{2 n-4}-1}{q+1}+d+\left(n_{+}+n_{-}\right) \frac{q^{2 n-3}-q^{2 n-4}}{2} .
\end{gathered}
$$

But $\varphi(\boldsymbol{d})=\varphi\left(\boldsymbol{d}^{\prime}\right)$, hence

$$
\left(a+\sum_{i} c_{i}-n_{-}\right)+\left(b+\sum_{i} c_{i}-n_{+}\right)=0 .
$$

4) From (25) and (26) it follows that

$$
n_{-}=a+\sum_{i} c_{i}, n_{+}=b+\sum_{i} c_{i}
$$

Now we define $W$ to be the (virtual) $\mathbb{F} G$-module with Brauer character $a \widehat{\alpha}_{n}+b \widehat{\beta}_{n}+$ $\sum_{i=1}^{(r-1) / 2} c_{i} \hat{\zeta}_{n}^{i}+d \cdot 1_{G}$. By Proposition [7.4

$\left.W\right|_{P_{1}}=\left(a \widehat{\alpha}_{n-1}+b \widehat{\beta}_{n-1}+\sum_{i} c_{i} \hat{\zeta}_{n-1}^{i}\right) \otimes 1_{T_{1}}+\left(a+\sum_{i} c_{i}\right) \widehat{\boldsymbol{B}}_{-}+\left(b+\sum_{i} c_{i}\right) \widehat{\boldsymbol{B}}_{+}+d \cdot 1_{G}$.

Then equalities (23), (24), (27), and (28) imply that $\left.V\right|_{P_{1}^{\prime}}=\left.W\right|_{P_{1}^{\prime}}$ and moreover $\left[V, Q_{1}\right]=\left[W, Q_{1}\right]$ as $P_{1}$-modules.

5) Next we consider a conjugate $T_{2}$ of $T_{1}$ that is contained in $L_{1}^{\prime}$. Since $T_{2}<P_{1}^{\prime}$, $V=W$ as $T_{2}$-modules. But $T_{1}$ and $T_{2}$ are conjugate, hence $V=W$ as $T_{1}$ modules. Now $T_{1}<P_{1}$, whence $\left[V, Q_{1}\right]=\left[W, Q_{1}\right]$ as $T_{1}$-modules. It follows that $C_{V}\left(Q_{1}\right)=C_{W}\left(Q_{1}\right)$ as $T_{1}$-modules. But $T_{1}$ acts trivially on $C_{W}\left(Q_{1}\right)$ by (28), so $T_{1}$ also acts trivially on $C_{V}\left(Q_{1}\right)$.

Define $e:=1$ if $\ell \mid(q+1)$ and 0 otherwise. By Corollary[7.5, $\widehat{\beta}_{n-1}=\phi_{n-1}+e \cdot 1_{L_{1}^{\prime}}$ where $\phi_{n-1} \in \operatorname{IBr}_{\ell}\left(L_{1}^{\prime}\right)$. Thus instead of (24) we have

$$
\left.C_{V}\left(Q_{1}\right)\right|_{L_{1}^{\prime}}=a \widehat{\alpha}_{n-1}+b \phi_{n-1}+\sum_{i=1}^{(r-1) / 2} c_{i} \hat{\zeta}_{n-1}^{i}+(d+e) \cdot 1_{L_{1}^{\prime}},
$$

where $d+e \geq 0$ since it is the multiplicity of $1_{L_{1}^{\prime}}$ in $\left.C_{V}\left(Q_{1}\right)\right|_{L_{1}^{\prime}}$. We have mentioned above that $a, b, c_{i} \geq 0$. Hence the trivial action of $T_{1}$ on $C_{V}\left(Q_{1}\right)$ (and (29)) implies that

$$
\left.C_{V}\left(Q_{1}\right)\right|_{L_{1}}=a \widehat{\alpha}_{n-1} \otimes 1_{T_{1}}+b \phi_{n-1} \otimes 1_{T_{1}}+\sum_{i=1}^{(r-1) / 2} c_{i} \hat{\zeta}_{n-1}^{i} \otimes 1_{T_{1}}+(d+e) \cdot 1_{L_{1}},
$$

and so

$$
\left.C_{V}\left(Q_{1}\right)\right|_{L_{1}}=a \widehat{\alpha}_{n-1} \otimes 1_{T_{1}}+b \widehat{\beta}_{n-1} \otimes 1_{T_{1}}+\sum_{i=1}^{(r-1) / 2} c_{i} \hat{\zeta}_{n-1}^{i} \otimes 1_{T_{1}}+d \cdot 1_{L_{1}} .
$$

It follows from (23), (30), and (28) that $V=W$ as $P_{1}$-modules. 
Proposition 8.2. Let $G=S p_{2 n}(q)$ with $n \geq 3,(n, q) \neq(3,2),(4,2)$ and let $V$ be an irreducible $\mathbb{F} G$-module. Assume that $V$ satisfies $\left(\mathcal{W}_{2}^{+}\right)$. Then there is a formal sum $W$ of trivial and (irreducible) linear-Weil modules of $G$ such that $\left.V\right|_{P_{1}^{\prime}}=\left.W\right|_{P_{1}^{\prime}}$.

Proof. Write $q-1=\ell^{k} r$ with $(r, \ell)=1$. Let $\varphi$ be the Brauer character of $V$.

1) Without loss we may assume that $V$ is nontrivial. By Proposition 5.7 and Theorem [5.11, $V$ satisfies the conclusion of Theorem[5.11(ii). It follows that

$$
\left.V\right|_{P_{1}^{\prime}}=\left.C_{V}\left(Q_{1}\right) \oplus\left(m \widehat{\boldsymbol{C}}+n_{+} \widehat{\boldsymbol{A}}_{+}+n_{-} \widehat{\boldsymbol{A}}_{-}\right)\right|_{P_{1}^{\prime}}
$$

for some nonnegative integers $m, n_{+}, n_{-}$(in the notation of 877). According to Proposition [7.9, all nontrivial composition factors of $\left.\widehat{\boldsymbol{C}}\right|_{L_{1}^{\prime}},\left.\widehat{\boldsymbol{A}}_{+}\right|_{L_{1}^{\prime}}$, and $\left.\widehat{\boldsymbol{A}}_{-}\right|_{L_{1}^{\prime}}$ are contained in $\left.\hat{\rho}_{n}^{1}\right|_{L_{1}^{\prime}}$ and $\left.\hat{\rho}_{n}^{2}\right|_{L_{1}^{\prime}}$, and therefore they are linear-Weil modules of $L_{1}^{\prime}$ by Proposition 7.7. The same also holds for $\left.C_{V}\left(Q_{1}\right)\right|_{L_{1}^{\prime}}$ by Lemma 2.1. By Corollary 7.10 applied to $L_{1}^{\prime}$,

$$
\left.C_{V}\left(Q_{1}\right)\right|_{L_{1}^{\prime}}=a \hat{\rho}_{n-1}^{1}+b \hat{\rho}_{n-1}^{2}+\sum_{i=1}^{(r-1) / 2} c_{i} \hat{\tau}_{n-1}^{i}+d \cdot 1_{L_{1}^{\prime}}
$$

for some $a, b, c_{i}, d \in \mathbb{Z}$. Notice that $a, b, c_{i} \geq 0$. Define $W$ to be the (virtual) $\mathbb{F} G$-module with Brauer character

$$
a \hat{\rho}_{n}^{1}+b \hat{\rho}_{n}^{2}+\sum_{i=1}^{(r-1) / 2} c_{i} \hat{\tau}_{n}^{i}+\left(d-a-b-2 \sum_{i=1}^{(r-1) / 2} c_{i}\right) \cdot 1_{G} .
$$

Then Proposition 7.9 and (31), (32) imply that

$$
\left.\psi\right|_{P_{1}^{\prime}}=x \widehat{\boldsymbol{C}}+y_{-} \widehat{\boldsymbol{A}}_{-}+y_{+} \widehat{\boldsymbol{A}}_{+},
$$

where $\psi$ is the Brauer character of the (virtual) $\mathbb{F} G$-module $W-V, c:=\sum_{i=1}^{(r-1) / 2} c_{i}$, $x:=a+b+2 c-m, y_{-}:=a+c-n_{-}$, and $y_{+}:=b+c-n_{+}$.

2) Here we compute $\psi(\boldsymbol{t})$ and $\psi\left(\boldsymbol{t}^{\prime}\right)$ for transvections $\boldsymbol{t} \in Z_{1}$ and $\boldsymbol{t}^{\prime} \in L_{1}^{\prime}$. Notice that for any $h \in L_{1}^{\prime}, \boldsymbol{C}(h)+1$ is just the number of $h$-fixed points on $\left\langle e_{2}, \ldots, e_{n}, f_{2}, \ldots, f_{n}\right\rangle_{\mathbb{F}_{q}}$; in particular, $\boldsymbol{C}\left(\boldsymbol{t}^{\prime}\right)=q^{2 n-3}-1$. Furthermore, any $\lambda \in \mathcal{O}_{1}$ is trivial on $Z_{1}$, whence $\boldsymbol{C}(\boldsymbol{t})=\boldsymbol{C}(1)$. By Proposition 7.9 and Corollary 7.8

$$
\begin{aligned}
\boldsymbol{A}_{-}(\boldsymbol{t}) & =\rho_{n}^{1}(\boldsymbol{t})-1-\rho_{n-1}^{1}(1)-\boldsymbol{C}(1)=\left(-q^{2 n-2}+q^{n-1}\right) / 2, \\
\boldsymbol{A}_{-}\left(\boldsymbol{t}^{\prime}\right) & =\rho_{n}^{1}\left(\boldsymbol{t}^{\prime}\right)-1-\rho_{n-1}^{1}\left(\boldsymbol{t}^{\prime}\right)-\boldsymbol{C}\left(\boldsymbol{t}^{\prime}\right)=\left(q^{2 n-2}-q^{2 n-3}\right) / 2 .
\end{aligned}
$$

Similarly,

$$
\boldsymbol{A}_{+}(\boldsymbol{t})=\left(-q^{2 n-2}-q^{n-1}\right) / 2, \boldsymbol{A}_{+}\left(\boldsymbol{t}^{\prime}\right)=\left(q^{2 n-2}-q^{2 n-3}\right) / 2 .
$$

Now we have

$$
\begin{aligned}
& \psi(\boldsymbol{t})=x\left(q^{2 n-2}-1\right)+y_{-}\left(-q^{2 n-2}+q^{n-1}\right) / 2+y_{+}\left(-q^{2 n-2}-q^{n-1}\right) / 2 \\
& \psi\left(\boldsymbol{t}^{\prime}\right)=x\left(q^{2 n-3}-1\right)+y_{-}\left(q^{2 n-2}-q^{2 n-3}\right) / 2+y_{+}\left(q^{2 n-2}-q^{2 n-3}\right) / 2 .
\end{aligned}
$$

But $\psi(\boldsymbol{t})=\psi\left(\boldsymbol{t}^{\prime}\right)$, hence

$$
2 x\left(q^{n-1}-q^{n-2}\right)=y_{-}\left(2 q^{n-1}-q^{n-2}-1\right)+y_{+}\left(2 q^{n-1}-q^{n-2}+1\right) .
$$

3) Next we compute $\psi(\boldsymbol{d}), \psi\left(\boldsymbol{d}^{\prime}\right)$, and $\psi\left(\boldsymbol{d}^{\prime \prime}\right)$ for double transvections $\boldsymbol{d}=\boldsymbol{t} \boldsymbol{t}^{\prime}$ (where $\boldsymbol{t}$ and $\boldsymbol{t}^{\prime}$ are as in 2)), $\boldsymbol{d}^{\prime} \in L_{1}^{\prime}$, and $\boldsymbol{d}^{\prime \prime}=\operatorname{diag}\left(\left(\begin{array}{llll}1 & 0 & 1 & 1 \\ 0 & 1 & 1 & 0 \\ 0 & 0 & 1 & 0 \\ 0 & 0 & 0 & 1\end{array}\right), I_{2 n-4}\right)$ (in 
the basis $\left.\left(e_{1}, e_{2}, f_{1}, f_{2}, e_{3}, \ldots, e_{n}, f_{3}, \ldots, f_{n}\right)\right)$. Observe that $\boldsymbol{d}^{\prime \prime} \in Q_{1} \backslash Z_{1}$, whence $\boldsymbol{C}\left(\boldsymbol{d}^{\prime \prime}\right)=-1$. Furthermore, $\boldsymbol{C}(\boldsymbol{d})=\boldsymbol{C}\left(\boldsymbol{t}^{\prime}\right)=q^{2 n-3}-1$, and $\boldsymbol{C}\left(\boldsymbol{d}^{\prime}\right)=q^{2 n-4}-1$. By Proposition 7.9 and Corollary 7.8

$$
\begin{aligned}
\boldsymbol{A}_{-}(\boldsymbol{d}) & =\rho_{n}^{1}(\boldsymbol{d})-1-\rho_{n-1}^{1}\left(\boldsymbol{t}^{\prime}\right)-\boldsymbol{C}(\boldsymbol{d})=-q^{2 n-3} / 2 \\
\boldsymbol{A}_{-}\left(\boldsymbol{d}^{\prime}\right) & =\rho_{n}^{1}\left(\boldsymbol{d}^{\prime}\right)-1-\rho_{n-1}^{1}\left(\boldsymbol{d}^{\prime}\right)-\boldsymbol{C}\left(\boldsymbol{d}^{\prime}\right)=\left(q^{2 n-3}-q^{2 n-4}\right) / 2 \\
\boldsymbol{A}_{-}\left(\boldsymbol{d}^{\prime \prime}\right) & =\rho_{n}^{1}\left(\boldsymbol{d}^{\prime \prime}\right)-1-\rho_{n-1}^{1}(1)-\boldsymbol{C}\left(\boldsymbol{d}^{\prime \prime}\right)=q^{n-1} / 2
\end{aligned}
$$

Similarly,

$$
\boldsymbol{A}_{+}(\boldsymbol{d})=-q^{2 n-3} / 2, \boldsymbol{A}_{+}\left(\boldsymbol{d}^{\prime}\right)=\left(q^{2 n-3}-q^{2 n-4}\right) / 2, \boldsymbol{A}_{+}\left(\boldsymbol{d}^{\prime \prime}\right)=-q^{n-1} / 2 .
$$

Now we obtain

$$
\begin{aligned}
& \psi(\boldsymbol{d})=x\left(q^{2 n-3}-1\right)-\left(y_{-}+y_{+}\right) q^{2 n-3} / 2 \\
& \psi\left(\boldsymbol{d}^{\prime}\right)=x\left(q^{2 n-4}-1\right)+\left(y_{-}+y_{+}\right)\left(q^{2 n-3}-q^{2 n-4}\right) / 2 \\
& \psi\left(\boldsymbol{d}^{\prime \prime}\right)=-x+\left(y_{-}-y_{+}\right) q^{n-1} / 2 .
\end{aligned}
$$

But $\psi(\boldsymbol{d})=\psi\left(\boldsymbol{d}^{\prime}\right)=\psi\left(\boldsymbol{d}^{\prime \prime}\right)$, hence

$$
x=\frac{\left(y_{-}+y_{+}\right)(2 q-1)}{2(q-1)}, 2 x q^{n-3}+y_{-}\left(q^{n-2}-q^{n-3}-1\right)+y_{+}\left(q^{n-2}-q^{n-3}+1\right)=0 .
$$

The equations (34) and (35) yield $x=y_{+}=y_{-}=0$. Thus $\left.\psi\right|_{P_{1}^{\prime}}=0$ by (33), i.e. $V=W$ as $P_{1}^{\prime}$-modules.

Now we can prove an analogue of Proposition 8.1 for linear-Weil characters.

Proposition 8.3. Let $G=S p_{2 n}(q)$ with $n \geq 3,(n, q) \neq(3,2),(4,2)$ and let $V$ be an irreducible $\mathbb{F} G$-module. Assume that $V$ satisfies $\left(\mathcal{W}_{2}^{+}\right)$. Then there is a formal sum $W$ of trivial and (irreducible) linear-Weil modules of $G$ such that $\left.V\right|_{P_{1}}=\left.W\right|_{P_{1}}$.

Proof. 1) By Proposition 8.2 there is a formal sum $W$ of trivial and (irreducible) linear-Weil modules of $G$ such that $\left.V\right|_{P_{1}^{\prime}}=\left.W\right|_{P_{1}^{\prime}}$. We will keep all the notation used in the proof of Proposition 8.2. For any $\lambda \in \operatorname{IBr}_{\ell}\left(Q_{1}\right)$ let $V_{\lambda}$ denote the $\lambda$ homogeneous component of $V$. Clearly, $V=C_{V}\left(Q_{1}\right) \oplus V_{1} \oplus V_{2}^{+} \oplus V_{2}^{-}$as $P_{1}$-modules, where $V_{1}=\sum_{\lambda \in \mathcal{O}_{1}} V_{\lambda}, V_{2}^{\varepsilon}=\sum_{\lambda \in \mathcal{O}_{2}^{\varepsilon}} V_{\lambda}$. Similarly, $W=C_{W}\left(Q_{1}\right) \oplus W_{1} \oplus W_{2}^{+} \oplus W_{2}^{-}$. The proof of Proposition 8.2 also shows that $V_{2}^{\varepsilon}=W_{2}^{\varepsilon}$ as $P_{1}$-modules for $\varepsilon= \pm$. It remains to prove that $V_{1}=W_{1}$ and $C_{V}\left(Q_{1}\right)=C_{W}\left(Q_{1}\right)$ as $P_{1}$-modules. Now (32) implies

$$
\left.C_{V}\left(Q_{1}\right)\right|_{L_{1}}=\hat{\rho}_{n-1}^{1} \otimes \alpha+\hat{\rho}_{n-1}^{2} \otimes \beta+\sum_{i=1}^{(r-1) / 2} \hat{\tau}_{n-1}^{i} \otimes \mu_{i}+1_{L_{1}^{\prime}} \otimes \eta,
$$

where $\alpha$, resp. $\beta, \mu_{i}$, are Brauer characters of $T_{1}$ of degree $a$, resp. $b, c_{i}$; furthermore, $\eta$ is a virtual Brauer character of $T_{1}$ of degree $d$. On the other hand, the choice of $W$ and Proposition 7.9 yield

$$
\begin{aligned}
\left.C_{W}\left(Q_{1}\right)\right|_{L_{1}}= & \left(a \hat{\rho}_{n-1}^{1}+b \hat{\rho}_{n-1}^{2}+\sum_{i=1}^{(r-1) / 2} c_{i} \hat{\tau}_{n-1}^{i}+\left(d-2 \sum_{i=1}^{(r-1) / 2} c_{i}\right) 1_{L_{1}^{\prime}}\right) \\
& \otimes 1_{T_{1}}+\sum_{i=1}^{(r-1) / 2} c_{i} 1_{L_{1}^{\prime}} \otimes\left(\delta_{i}+\delta_{-i}\right) .
\end{aligned}
$$

One can also show that there are Brauer characters $\gamma$ and $\gamma^{\prime}$ of $P_{1}$ that are trivial on $P_{1}^{\prime}$ and of same degree $m$ such that

$$
\left.V_{1}\right|_{P_{1}}=\boldsymbol{C} \gamma,\left.W_{1}\right|_{P_{1}}=\boldsymbol{C} \gamma^{\prime} .
$$


Observe that

$$
\boldsymbol{C} \gamma=\operatorname{Ind}_{K_{\lambda}}^{P_{1}}\left(\left.\hat{\lambda} \cdot \gamma\right|_{K_{\lambda}}\right), \boldsymbol{C} \gamma^{\prime}=\operatorname{Ind}_{K_{\lambda}}^{P_{1}}\left(\left.\hat{\lambda} \cdot \gamma^{\prime}\right|_{K_{\lambda}}\right)
$$

for $\lambda \in \mathcal{O}_{1}$.

2) Consider the following two subgroups of $G$ :

$$
R_{1}=\operatorname{Stab}_{G}\left(\left\langle e_{1}\right\rangle_{\mathbb{F}_{q}}, e_{2}, f_{2}\right), R_{2}=\operatorname{Stab}_{G}\left(\left\langle e_{2}\right\rangle_{\mathbb{F}_{q}}, e_{1}, f_{1}\right) .
$$

Since $R_{2}<P_{1}^{\prime}, V=W$ as $R_{2}$-modules. But $R_{1}$ and $R_{2}$ are conjugate, whence $V=W$ as $R_{1}$-modules. Since $R_{1}<P_{1}$, we see that $C_{V}\left(Q_{1}\right) \oplus V_{1}=C_{W}\left(Q_{1}\right) \oplus W_{1}$ as $R_{1}$-modules. Observe that $R_{1}=\widetilde{Q}_{1}: \widetilde{L}_{1}$ plays the role of the $P_{1}^{\prime}$-subgroup in $\operatorname{Stab}_{G}\left(e_{2}, f_{2}\right) \simeq S p_{2 n-2}(q)$, with $\widetilde{Q}_{1}=\left[q^{2 n-3}\right]$ and $\widetilde{L}_{1}:=\operatorname{Stab}_{R_{1}}\left(\left\langle f_{1}\right\rangle_{\mathbb{F}_{q}}\right) \simeq$ $\operatorname{Sp}_{2 n-4}(q) \times \mathbb{Z}_{q-1}$.

Fix the $Q_{1}$-character $\lambda:=\lambda_{B}:[A, C] \mapsto(-1)^{\operatorname{tr}_{\mathbb{F}_{q} / \mathbb{F}_{2}}\left({ }^{t} b J_{n-1} A\right)}$, where $b=e_{3}$ (here we identify $\mathbb{F}_{q}^{2 n-2}$ with $\left.\left\langle e_{2}, \ldots, e_{n}, f_{2}, \ldots, f_{n}\right\rangle_{\mathbb{F}_{q}}\right)$. Let $\pi:=\left.\lambda\right|_{\widetilde{Q}_{1}}$. Define $q^{2}$ distinct $Q_{1}$-characters $\lambda_{x, y}:=\lambda_{b_{x, y}}$, where $b_{x, y}:=x e_{2}+e_{3}+y f_{2}$ for $x, y \in \mathbb{F}_{q}$. Then these $\lambda_{x, y}$ are exactly the $Q_{1}$-characters from $\mathcal{O}_{1}$ that are equal to $\pi$ when restricted to $\widetilde{Q}_{1}$. Furthermore, $R_{\pi}:=\operatorname{Stab}_{R_{1}}(\pi)=\widetilde{Q}_{1}:\left(J: T^{\prime}\right)$, where $J:=\left\{g \in \widetilde{L}_{1} \mid g(b)=b\right\}$ and

$$
T^{\prime}:=\left\{\boldsymbol{z}:=\operatorname{diag}\left(z, 1, z, I_{n-3}, 1, z^{-1}, I_{n-3}, z^{-1}\right) \mid z \in \mathbb{F}_{q}^{\bullet}\right\}
$$

(in the basis $\left.\left(e_{1}, e_{2}, \ldots, e_{n}, f_{2}, \ldots, f_{n}, f_{1}\right)\right)$. By Lemma 2.3, the $\pi$-homogeneous components $\widetilde{V}_{\pi}$ (of $\left.\left(C_{V}\left(Q_{1}\right) \oplus V_{1}\right)\right|_{\widetilde{Q}_{1}}$ ) and $\widetilde{W}_{\pi}$ (of $\left.\left(C_{W}\left(Q_{1}\right) \oplus W_{1}\right)\right|_{\widetilde{Q}_{1}}$ ) are equal as $R_{\pi}$-modules.

It is not difficult to see that $\widetilde{V}_{\pi}=\sum_{x, y \in \mathbb{F}_{q}} V_{\lambda_{x, y}}$ (and $\widetilde{W}_{\pi}=\sum_{x, y \in \mathbb{F}_{q}} W_{\lambda_{x, y}}$ ) affords the $Q_{1}$-character $m \sum_{x, y \in \mathbb{F}_{q}} \lambda_{x, y}$, and $\boldsymbol{z} \in T^{\prime}$ maps $\lambda_{x, y}$ to $\lambda_{z^{-1} x, z^{-1} y}$. Let $\boldsymbol{z} \in T^{\prime}$ be any nontrivial $\ell^{\prime}$-element. Then $\boldsymbol{z}$ acts regularly on $\left\{\lambda_{x, y} \mid(0,0) \neq\right.$ $\left.(x, y) \in \mathbb{F}_{q}^{2}\right\}$. Hence the trace of $\boldsymbol{z}$ acting on $\widetilde{V}_{\pi}$ equals the trace of $\boldsymbol{z}$ acting on $V_{\lambda}$. Recall that $z \in I_{\lambda}$ and $\operatorname{Ker}(\hat{\lambda})>I_{\lambda}$. Therefore, (38) and (39) imply that the trace of $\boldsymbol{z}$ acting on $V_{\lambda}$ is $\gamma(\boldsymbol{z})$. Thus the trace of $\boldsymbol{z}$ acting on $\widetilde{V}_{\pi}$ equals $\gamma(\boldsymbol{z})$. Similarly, the trace of $\boldsymbol{z}$ acting on $\widetilde{W}_{\pi}$ equals $\gamma^{\prime}(\boldsymbol{z})$. Since $\widetilde{V}_{\pi}=\widetilde{W}_{\pi}$ as $R_{\pi}$-modules, it follows that $\gamma(\boldsymbol{z})=\gamma^{\prime}(\boldsymbol{z})$, for any $\ell^{\prime}$-element $1 \neq \boldsymbol{z} \in T^{\prime}$. But $\gamma(1)=\gamma^{\prime}(1)=m$. Hence $\left.\gamma\right|_{T^{\prime}}=\left.\gamma^{\prime}\right|_{T^{\prime}}$ (as Brauer characters). Finally, observe that $P_{1}=P_{1}^{\prime} T^{\prime}$, and $\gamma$ and $\gamma^{\prime}$ are actually $P_{1} / P_{1}^{\prime}$-characters. Consequently, $\gamma=\gamma^{\prime}$, and so $V_{1}=W_{1}$ as $P_{1}$-modules, because of (38).

$3)$ The results of 2) imply that $C_{V}\left(Q_{1}\right)=C_{W}\left(Q_{1}\right)$ as $R_{1}$-modules. Since $\widetilde{Q}_{1}<$ $Q_{1}$, we can also say that $C_{V}\left(Q_{1}\right)=C_{W}\left(Q_{1}\right)$ as modules over $\widetilde{L}_{1}=\widetilde{L}_{1}^{\prime} \times T_{1} \simeq$ $S p_{2 n-4}(q) \times \mathbb{Z}_{q-1}$. By Corollary 7.10 there is a unique nontrivial composition factor $\rho$ of $\hat{\rho}_{n-2}^{2}$ (as an $\widetilde{L}_{1}^{\prime}$-character). By Proposition[7.7, Corollary [7.10, and (36), the $\rho$-homogeneous component of $\left.C_{V}\left(Q_{1}\right)\right|_{\widetilde{L}_{1}^{\prime}}$ affords the $T_{1}$-character

$$
\left(1+(q+1) \frac{\ell^{k}-1}{2}\right) \alpha+(q+1)\left(1+\frac{\ell^{k}-1}{2}\right) \beta+(q+1) \ell^{k} \sum_{i=1}^{(r-1) / 2} \mu_{i},
$$

and the $\rho$-homogeneous component of $\left.C_{W}\left(Q_{1}\right)\right|_{\tilde{L}_{1}^{\prime}}$ affords the $T_{1}$-character

$$
\left(\left(1+(q+1) \frac{\ell^{k}-1}{2}\right) a+(q+1)\left(1+\frac{\ell^{k}-1}{2}\right) b+(q+1) \ell^{k} \sum_{i=1}^{(r-1) / 2} c_{i}\right) 1_{T_{1}} .
$$


These last two $T_{1}$-characters are equal, and $\alpha(1)=a \geq 0, \beta(1)=b \geq 0, \mu_{i}(1)=$ $c_{i} \geq 0$. Hence

$$
\alpha=a \cdot 1_{T_{1}}, \beta=b \cdot 1_{T_{1}}, \mu_{i}=c_{i} \cdot 1_{T_{1}} .
$$

In this case, the equality of $\widetilde{L}_{1}$-modules $C_{V}\left(Q_{1}\right)$ and $C_{W}\left(Q_{1}\right)$ reduces to

$$
\eta=\left(d-2 \sum_{i=1}^{(r-1) / 2} c_{i}\right) 1_{T_{1}}+\sum_{i=1}^{(r-1) / 2} c_{i}\left(\delta_{i}+\delta_{-i}\right)
$$

Now (36), (37), (40), and (41) imply that $C_{V}\left(Q_{1}\right)=C_{W}\left(Q_{1}\right)$ as $P_{1}$-modules, as desired.

The main result of this section is the following

Theorem 8.4. Let $G=S p_{2 n}(q)$ with $n \geq 3,(n, q) \neq(3,2),(4,2)$, and let $V$ be an irreducible $\mathbb{F} G$-module.

(i) Assume that $V$ satisfies $\left(\mathcal{W}_{2}^{+}\right)$. Then there is a formal sum $W$ of trivial and (irreducible) linear-Weil modules of $G$ such that $V=W$ as $P_{1}$-modules and also as $H_{d}$-modules for $1 \leq d \leq n / 2$.

(ii) Assume that $V$ satisfies $\left(\mathcal{W}_{2}^{-}\right)$. Then there is a formal sum $W$ of trivial and (irreducible) unitary-Weil modules of $G$ such that $V=W$ as $P_{1}$-modules and also as $H_{d}$-modules for $1 \leq d \leq n / 2$.

Proof. (i) By Proposition 8.3, there is a formal sum $W$ of trivial and (irreducible) linear-Weil modules of $G$ such that $\left.V\right|_{P_{1}}=\left.W\right|_{P_{1}}$. We need to show that $V=W$ as $H_{d}$-modules for $1 \leq d \leq n / 2$. Recall that $H_{d}=A \times B$, where $A \simeq S p_{2 n-2 d}(q)$ and $B \simeq S p_{2 d}(q)$. We may conjugate $H_{d}$ so that $A \leq L_{1}^{\prime}<P_{1}$. Then all composition factors of $\left.W\right|_{A}$ are either trivial or linear-Weil by Lemma 3.7. The same is true for $\left.V\right|_{A}$, since $V=W$ as $P_{1}$-modules and $A<P_{1}$. The same also holds for $\left.W\right|_{B}$ and $\left.V\right|_{B}$, since $B$ is $G$-conjugate to a subgroup of $L_{1}^{\prime}$. For the virtual module $U:=W-V$, we may therefore write

$$
\left.U\right|_{H_{d}}=\sum_{j=1}^{(r+5) / 2} M_{j} \otimes N_{j},
$$

where $N_{j}$ runs over the set $\mathfrak{W}_{+}(B)$ consisting of $1_{B}$ and $(r+3) / 2$ distinct irreducible linear-Weil modules of $B$, and each $M_{j}$ is a formal sum of trivial and linear-Weil modules of $A$.

Consider the $P_{1}$-parabolic subgroup $P_{1}^{*}$ of $A$. We may conjugate $H_{d}$ so that $P_{1}^{*} \times B<P_{1}$. Since $\left.U\right|_{P_{1}}=0$, we have

$$
0=\left.U\right|_{P_{1}^{*} \times B}=\sum_{j=1}^{(r+5) / 2}\left(\left.M_{j}\right|_{P_{1}^{*}}\right) \otimes N_{j} .
$$

But the $N_{j}$ are all distinct irreducible $B$-modules, hence the last equality implies that $\left.M_{j}\right|_{P_{1}^{*}}=0$ for each $j$. By Lemma 7.11 applied to $B, M_{j}=0$ for all $j$. Consequently, $U=0$ as $H_{d}$-modules, i.e. $V=W$ as $H_{d}$-modules.

(ii) Similar to (i). 


\section{Restrictions to $P_{j}, 2 \leq j \leq n-1$}

In this section we consider the restriction to any parabolic subgroup $P_{j}$ with $2 \leq j \leq n-1$. If $V$ is a (virtual) $\mathbb{F} G$-module satisfying $\left(\mathcal{W}_{2}^{\varepsilon}\right)$, then we can write

$$
\left.V\right|_{P_{j}}=C_{V}\left(Q_{j}\right) \oplus V_{0} \oplus V_{1} \oplus V_{2} .
$$

Here $Z_{j}$ acts trivially on $V_{0}$ and all $Q_{j}$-characters on $V_{0}$ are nontrivial. Furthermore, all $Z_{j}$-characters occuring in $V_{1}$, resp. in $V_{2}$, belong to $\mathcal{O}_{1}$, resp. to $\mathcal{O}_{2}^{\varepsilon}$. For any $Z_{j}$-character $\lambda$, let $K_{\lambda}:=\operatorname{Stab}_{P_{j}}(\lambda)$ and $I_{\lambda}:=\operatorname{Stab}_{L_{j}}(\lambda)$.

Throughout this section, we assume that $G=S p_{2 n}(q)$ with $n \geq 3,(n, q) \neq$ $(3,2),(4,2)$, and that $V$ is an irreducible $\mathbb{F} G$-module satisfying $\mathcal{W}_{2}^{\varepsilon}$ for some $\varepsilon$. By Theorem 8.4 there is a formal sum $W$ of trivial and Weil modules of $G$ such that $V=W$ as $P_{1}$-modules and also as $H_{d}$-modules for $1 \leq d \leq n / 2$.

First we focus on the summand $V_{2}$ in the case $j=2$. We keep the notation introduced in $\S 3$ before Lemma 3.1 .

Lemma 9.1. Let $\lambda=\lambda_{B}$ be a nontrivial linear character of $Z_{2}$ that belongs to $\mathcal{O}_{2}^{\varepsilon}$. Suppose that $x \in I_{\lambda}$ induces an element of odd order in $O\left(q_{B}\right)$. Then $x$ acts trivially on the complete inverse image of $C_{Q_{2} / Z_{2}}(x)$ in $Q_{2} / \operatorname{Ker}\left(\lambda_{B}\right)$.

Proof. Let $g=[A, C] \in Q_{2}$ be centralized by $x$ modulo $Z_{2}$. We need to show that $h:=x g x^{-1} g^{-1} \in \operatorname{Ker}\left(\lambda_{B}\right)$. Since $x$ stabilizes $\lambda_{B}, x=\operatorname{diag}\left(X, Y,{ }^{t} X^{-1}\right)$ with $X \in O\left(q_{B}\right)$ and $Y \in S p_{2 n-4}(q)$. By assumption, $X$ has odd order say $m$. Since $\operatorname{rank}\left(q_{B}\right)=2, D:=B+{ }^{t} B=\left(\begin{array}{ll}0 & d \\ d & 0\end{array}\right)$ for some $d \in \mathbb{F}_{q}^{\bullet}$. The condition $X \in O\left(q_{B}\right)$ implies that ${ }^{t} X D X=D$ and ${ }^{t} X B X-B \in \mathcal{H}_{2}^{0}(q)$, i.e. ${ }^{t} X B X-B=\left(\begin{array}{ll}0 & b \\ b & 0\end{array}\right)$ for some $b \in \mathbb{F}_{q}$, whence ${ }^{t} X B X=B+b d^{-1} D$. It follows that $B={ }^{t} X^{m} B X^{m}=$ $B+m b d^{-1} D$, i.e. $m b d^{-1} D=0$. But $m$ is odd, hence $b=0$, and ${ }^{t} X B X=B$.

Since $x g x^{-1} \equiv g\left(\bmod Z_{2}\right)$, we have $h=\left[0, X C^{t} X+C\right]$. Observe that

$$
\operatorname{Tr}\left(B\left(X C^{t} X+C\right)\right)=\operatorname{Tr}\left({ }^{t} X B X \cdot C\right)+\operatorname{Tr}(B C)=\operatorname{Tr}(B C)+\operatorname{Tr}(B C)=0 .
$$

Thus $\lambda_{B}(h)=1$ as stated.

Lemma 9.2. Let $\lambda=\lambda_{B}$ be a nontrivial linear character of $Z_{2}$ that belongs to $\mathcal{O}_{2}^{\varepsilon}$. Then there is a unique irreducible $\ell$-Brauer character $\mu$ of $Q_{2}$ of degree $q^{2 n-4}$ such that $\left.\mu\right|_{Z_{2}}=\mu(1) \lambda$. Moreover $\mu$ extends to a Brauer character $\tilde{\mu}$ of $K_{\lambda}$. If $x \in I_{\lambda}$ is an $\ell^{\prime}$-element that induces an element of odd order in $O\left(q_{B}\right)$, then $\tilde{\mu}(x) \neq 0$.

Proof. One can check that $\left(Z_{2}: \operatorname{Ker}\left(\lambda_{B}\right)\right)=\left(Q_{2}^{\prime}:\left(\operatorname{Ker}\left(\lambda_{B}\right) \cap Q_{2}^{\prime}\right)\right)=2$ and moreover $Q_{2} / \operatorname{Ker}\left(\lambda_{B}\right)$ is an extraspecial 2 -group of order $2 q^{4 n-8}$. Hence there exists a unique $\mu \in \operatorname{IBr}_{\ell}\left(Q_{2}\right)$ of degree $q^{2 n-4}$ such that $\left.\mu\right|_{Z_{2}}=\mu(1) \lambda$.

Assume $\varepsilon=+$. Using (77) and Corollary [7.8 one can show that

$\left.\rho_{n}^{1}\right|_{Z_{2}}=\left(q^{2 n-3}+q^{2 n-4}+\frac{\left(q^{n-2}-q\right)\left(q^{n-2}+1\right)}{2(q-1)}\right) \cdot 1_{Z_{2}}+\frac{q^{2 n-4}-q^{n-2}}{2} \omega_{1}+q^{2 n-4} \omega_{2}^{+}$.

Thus the multiplicity of $\lambda$ in $\left.\rho_{n}^{1}\right|_{Z_{2}}$ is $q^{2 n-4}$. Let $A$ be an $\mathbb{F} G$-module that affords the unique nontrivial irreducible constituent of $\hat{\rho}_{n}^{1}$ (cf. Corollary 17.10). Then $A_{\lambda}$ has dimension $q^{2 n-4}$. Clearly, $Z_{2}$ acts on $A_{\lambda}$ with character $\mu(1) \lambda$, and both $Q_{2}$ and $K_{\lambda}$ act on $A_{\lambda}$. Thus $\mu$ extends to the $K_{\lambda}$-character $\tilde{\mu}$ of $A_{\lambda}$. Let $\nu \in \operatorname{Irr}\left(Q_{2}\right)$ be such that $\left.\nu\right|_{Z_{2}}=\nu(1) \lambda$. Applying the same argument to $\rho_{n}^{1}$ and $\nu$ we see that $\nu$ extends to a complex $K_{\lambda}$-character which we denote by the same letter $\nu$. It follows 
that $\hat{\nu}$ and $\tilde{\mu}$ are extensions of $\mu$ to $K_{\lambda}$, whence $\tilde{\mu}=\hat{\nu} \gamma$ for some linear character $\gamma$ of $K_{\lambda} / Q_{2}$. Now assume $x$ is as in the lemma. Then $x$ acts trivially on the complete inverse image of $C_{Q_{2} / Z_{2}}(x)$ in $Q_{2} / \operatorname{Ker}\left(\lambda_{B}\right)$ by Lemma 9.1, whence $\nu(x) \neq 0$ by Lemma2.4 (applied to $\left.Q:=Q_{2} / \operatorname{Ker}\left(\lambda_{B}\right)\right)$. Hence $|\tilde{\mu}(x)|=|\hat{\nu}(x) \gamma(x)|=|\nu(x)| \neq 0$, as stated.

If $\varepsilon=-$, then one can prove that

$$
\left.\alpha_{n}\right|_{Z_{2}}=\frac{\left(q^{n-2}-q\right)\left(q^{n-2}-1\right)}{2(q+1)} \cdot 1_{Z_{2}}+\frac{q^{2 n-4}-q^{n-2}}{2} \omega_{1}+q^{2 n-4} \omega_{2}^{-}
$$

and argue similarly.

Proposition 9.3. Under the above assumptions, $V_{2}=W_{2}$ as $P_{2}$-modules.

Proof. Since $Q_{2}<P_{1}, V=W$ as $Q_{2}$-modules. If $V$ or $W$ does not afford the orbit $\mathcal{O}_{2}^{\varepsilon}$ of $Q_{2}$-characters, then $V_{2}=W_{2}=0$ and we are done. So assume that $V$ and $W$ both afford $\mathcal{O}_{2}^{\varepsilon}$ and fix $\lambda=\lambda_{B} \in \mathcal{O}_{2}^{\varepsilon}$. Clearly, $\left.V_{2}\right|_{P_{2}}=\operatorname{Ind}_{K_{\lambda}}^{P_{2}}\left(V_{\lambda}\right)$ and similarly for $W_{2}$, hence it suffices to prove that $\varphi=\psi$, where $\varphi$, resp. $\psi$, is the Brauer $K_{\lambda}$-character afforded by the homogeneous component $V_{\lambda}$, resp. $W_{\lambda}$. Let $g \in K_{\lambda}$ be any $\ell^{\prime}$-element. Observe that there is a natural homomorphism $\pi: K_{\lambda} \rightarrow O\left(q_{B}\right)<G L\left(\left\langle e_{1}, e_{2}\right\rangle_{\mathbb{F}_{q}}\right)$ with $\operatorname{Ker}(\pi) \simeq Q_{2}: S p_{2 n-4}(q)$.

1) First we consider the case when $\pi(g)$ has even order in $O\left(q_{B}\right)$. Since $O\left(q_{B}\right)$ is a dihedral group of order $2(q-\varepsilon), \pi(g)$ is an involution and hence it fixes a nonzero vector in $\left\langle e_{1}, e_{2}\right\rangle_{\mathbb{F}_{q}}$. Setting $X=Q_{2}\langle g\rangle$, we see that $X$ is contained in a conjugate of $P_{1}$. Hence $V=W$ as $X$-modules, and so $V_{\lambda}=W_{\lambda}$ as $X$-modules by Lemma 2.3 (notice that $X$ preserves $\lambda$ ). It follows that $\varphi(g)=\psi(g)$.

2) Next we consider the case when $\pi(g)$ has odd order in $O\left(q_{B}\right)$, i.e. $\pi(g) \in$ $\Omega\left(q_{B}\right)$. Define $X:=\pi^{-1}\left(\Omega\left(q_{B}\right)\right)$. Clearly, the $Q_{2}$-module $V_{\lambda}$ is semisimple.

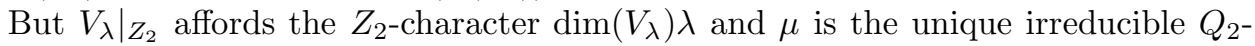
character with $\left.\mu\right|_{Z_{2}}=\mu(1) \lambda$ (see Lemma 9.2), so $\left.V_{\lambda}\right|_{Q_{2}}$ is a direct sum of some copies of a simple $\mathbb{F} Q_{2}$-module that affords $\mu$. Now $Q_{2} \triangleleft K_{\lambda}$ and $\mu$ extends to $\tilde{\mu}$ by Lemma 9.2 It follows that $\varphi=\tilde{\mu} \alpha$ for some Brauer character $\alpha$ of $K_{\lambda} / Q_{2}$. Similarly, $\psi=\tilde{\mu} \beta$ for some Brauer character $\beta$ of $K_{\lambda} / Q_{2}$.

Clearly, $K_{\lambda}=Q_{2}: I_{\lambda}$. We can embed $L_{2}$ into $H_{2}=\operatorname{Stab}_{G}\left(\left\langle e_{1}, e_{2}, f_{1}, f_{2}\right\rangle_{\mathbb{F}_{q}}\right)$, and write $X=Q_{2} Y$ with $Y=X \cap I_{\lambda}$. Observe that $Z_{2} Y<H_{2}$. Since $V=W$ as $H_{2}$-modules, $V=W$ as $Z_{2} Y$-modules. But $V_{\lambda}$ and $W_{\lambda}$ are the $\lambda$-homogeneous components of $\left.V\right|_{Z_{2}}$ and $\left.W\right|_{Z_{2}}$, and $Z_{2} Y$ preserves $\lambda$. It follows by Lemma 2.3 that $V_{\lambda}=W_{\lambda}$ as $Z_{2} Y$-modules; in particular, $\tilde{\mu}(h) \alpha(h)=\varphi(h)=\psi(h)=\tilde{\mu}(h) \beta(h)$ for any $\ell^{\prime}$-element $h \in Y$. Recall that $h \in I_{\lambda}$ and $h$ induces an element of odd order in $O\left(q_{B}\right)$, whence $\tilde{\mu}(h) \neq 0$ by Lemma 9.2 . This implies that $\alpha(h)=\beta(h)$, i.e. $\left.\alpha\right|_{Y}=\left.\beta\right|_{Y}$. Therefore $\varphi(g)=\tilde{\mu}(g) \alpha(y)=\tilde{\mu}(g) \beta(y)=\psi(g)$, where $y \in g Q_{2} \cap Y$.

An important role in further discussion is played by the following statement:

Proposition 9.4. Let $G=S p_{2 n}(q)$ with $n \geq 3$ and $(n, q) \neq(3,2), 2 \leq j \leq n$, and let $\chi$ be a Weil character of $G$. Assume $\lambda$ is a nontrivial linear character of $Q_{j} / Z_{j}$ that occurs in $\left.\chi\right|_{Q_{j}}$. Then $K_{\lambda}:=\operatorname{Stab}_{P_{j}}(\lambda)$ is contained in a conjugate of $P_{i}$ for some $i \leq j-1$.

Proof. Lemma 3.8 shows that it suffices to prove the proposition in the case $\chi \in$ $\left\{\rho_{n}^{1}, \rho_{n}^{2}, \alpha_{n}, \beta_{n}\right\}$. We keep the notation introduced at the beginning of $\S 3$. 
1) Recall that $Q_{j} / Z_{j}$ may be identified with the additive group $M_{j}:=$ $M_{2 n-2 j, j}\left(\mathbb{F}_{q}\right)$. The action via conjugation of the Levi subgroup $L_{j}=G L_{j}(q) \times$ $S p_{2 n-2 j}(q)$ on $M_{j}$ is given by the formula

$$
g X g^{-1}=C X^{t} A,
$$

where $g=\operatorname{diag}\left(A, C,{ }^{t} A^{-1}\right) \in L_{j}$ with $A \in G L\left(\left\langle e_{1}, \ldots, e_{j}\right\rangle_{\mathbb{F}_{q}}\right)$ and $C \in S:=$ $S p_{2 n-2 j}(q)$, and $X \in M_{j}$. It is easy to see that any linear character of $Q_{j} / Z_{j}$ is of the form $\lambda=\lambda_{B}: X \mapsto(-1)^{\operatorname{tr}_{\mathbb{F}_{q} / \mathbb{F}_{2}}\left(\operatorname{Tr}\left({ }^{t} B X\right)\right)}$ for some $B \in M_{j}$. Then the action of $g=\operatorname{diag}\left(A, C,{ }^{t} A^{-1}\right) \in L_{j}$ on $\operatorname{Irr}\left(Q_{j} / Z_{j}\right)$ is given by $g \circ \lambda_{B}=\lambda_{{ }^{t} C^{-1} B A^{-1}}$.

From now on we assume $\lambda=\lambda_{B}$ satisfies the assumption of the proposition. Let $\mathcal{O}$ denote the $L_{j}$-orbit of $\lambda$. Clearly, $|\mathcal{O}| \leq \chi(1)<q^{2 n}$.

2) Here we prove the statement in the case $\lambda=\lambda_{B}$ with $0<r:=\operatorname{rank}(B)<j$. Indeed, assume $g=\operatorname{diag}\left(A, C,{ }^{t} A^{-1}\right) \in I_{\lambda}$. Then $B={ }^{t} C^{-1} B A^{-1}$, and so ${ }^{t} A^{t} B=$ ${ }^{t} B C^{-1}$. Let ${ }^{t} B=\left[\boldsymbol{b}_{1}, \ldots, \boldsymbol{b}_{2 n-2 j}\right]$, where $\boldsymbol{b}_{k} \in \mathbb{F}_{q}^{j}$. The formula ${ }^{t} A^{t} B={ }^{t} B C^{-1}$ shows that ${ }^{t} A \boldsymbol{b}_{k} \in\left\langle\boldsymbol{b}_{1}, \ldots, \boldsymbol{b}_{2 n-2 j}\right\rangle_{\mathbb{F}_{q}}$ for all $k$. Thus ${ }^{t} A$ preserves a subspace of dimension $r$ of $\mathbb{F}_{q}^{j}$, whence $A$ preserves a subspace of dimension $j-r$ of $\mathbb{F}_{q}^{j}$. Returning to the specified basis of the natural module $M$ of $G$, we see that all elements $g=\operatorname{diag}\left(A, C,{ }^{t} A^{-1}\right) \in I_{\lambda}$ preserve a subspace $M^{\prime}$ (which depends only on $B$ ) of dimension $j-r$ of the totally isotropic subspace $\left\langle e_{1}, \ldots, e_{j}\right\rangle_{\mathbb{F}_{q}}$. Observe that $K_{\lambda}=Q_{j} I_{\lambda}$ and that $Q_{j}$ acts trivially on $\left\langle e_{1}, \ldots, e_{j}\right\rangle_{\mathbb{F}_{q}}$. It follows that $K_{\lambda}$ is contained in a conjugate of $P_{j-r}$.

3) Since $\lambda$ is nontrivial, $B \neq 0$. The rest of the proof is to show that $\operatorname{rank}(B) \neq j$. So we will now assume that $j=\operatorname{rank}(B) \leq 2 n-2 j$. We claim that there is a subspace $N^{\prime}$ of dimension $j$ of the natural module $N=\mathbb{F}_{q}^{2 n-2 j}$ for $S=S p_{2 n-2 j}(q)$ such that $\left|I_{\lambda}\right|=\left|\operatorname{Stab}_{S}\left(N^{\prime}\right)\right|$. Indeed, assume $g=\operatorname{diag}\left(A, C,{ }^{t} A^{-1}\right) \in I_{\lambda}$. Then $B={ }^{t} C^{-1} B A^{-1}$, and so ${ }^{t} C B=B A^{-1}$. Let $B=\left[\boldsymbol{b}_{1}^{\prime}, \ldots, \boldsymbol{b}_{j}^{\prime}\right]$, where $\boldsymbol{b}_{k}^{\prime} \in \mathbb{F}_{q}^{2 n-2 j}$. The formula ${ }^{t} C B=B A^{-1}$ shows that ${ }^{t} C \boldsymbol{b}_{k}^{\prime}$ lies in $N^{\prime}:=\left\langle\boldsymbol{b}_{1}^{\prime}, \ldots, \boldsymbol{b}_{j}^{\prime}\right\rangle_{\mathbb{F}_{q}}$ for all $k$, i.e. ${ }^{t} C \in \operatorname{Stab}_{S}\left(N^{\prime}\right)$. Moreover, since $\boldsymbol{b}_{1}^{\prime}, \ldots, \boldsymbol{b}_{j}^{\prime}$ are linearly independent, $A^{-1}$ (and so $A$ ) is completely determined by $B$ and $C$. Thus $\left|I_{\lambda}\right|=\left|\operatorname{Stab}_{S}\left(N^{\prime}\right)\right|$ as stated. In particular,

$$
|\mathcal{O}|=\left(L_{j}: I_{\lambda}\right)=\left|G L_{j}(q)\right| \cdot\left(S: \operatorname{Stab}_{S}\left(N^{\prime}\right)\right) .
$$

4) Here we handle the case $j \geq 3$. First assume that $j<2 n-2 j$. It is straightforward to check that $\left(S: \operatorname{Stab}_{S}\left(N^{\prime}\right)\right) \geq\left(q^{2 n-2 j}-1\right) /(q-1)$. Hence $|\mathcal{O}|=\left|G L_{j}(q)\right| \cdot\left(S: \operatorname{Stab}_{S}\left(N^{\prime}\right)\right)>q^{2 n}$, a contradiction. Now assume that $3 \leq j=2 n-2 j$. It follows that $n \geq 6$ and $j \geq 4$. In this case $S=\operatorname{Stab}_{S}\left(N^{\prime}\right)$, and $|\mathcal{O}|=\left|G L_{j}(q)\right|>q^{2 n+1}$, again a contradiction.

5) We are left with the case $j=2$. First assume that $n \geq 4$. Recall that $N^{\prime}$ is a 2-dimensional subspace of $N$, so $|\mathcal{O}|=\left|G L_{2}(q)\right| \cdot\left(S: S t a b_{S}\left(N^{\prime}\right)\right)$ equals $\left(q^{2 n-4}-1\right)\left(q^{2 n-4}-q^{2 n-5}\right)$ if $N^{\prime}$ is nondegenerate, and $\left(q^{2 n-4}-1\right)\left(q^{2 n-5}-q\right)$ if $N^{\prime}$ is degenerate. In both cases $|\mathcal{O}| \geq\left(q^{2 n-4}-1\right)\left(q^{3}-q\right)>q^{2 n-2}$. On the other hand, it is clear that $|\mathcal{O}|$ cannot exceed the multiplicity of $1_{Z_{2}}$ in $\left.\chi\right|_{Z_{2}}$, and, since $\chi \in\left\{\rho_{n}^{1}, \rho_{n}^{2}, \alpha_{n}, \beta_{n}\right\}$, this multiplicity is at most

$$
q^{2 n-3}+q^{2 n-4}+\left(q^{n-2}+q\right)\left(q^{n-2}-1\right) / 2(q-1)<q^{2 n-2} .
$$

Thus we get a contradiction if $n \geq 4$. Finally assume that $n=3$, so $q \geq 4$ by our assumption. Then $|\mathcal{O}|=\left(q^{2}-1\right)\left(q^{2}-q\right)$; meanwhile the multiplicity of $1_{Z_{2}}$ in $\left.\chi\right|_{Z_{2}}$ is at most $q^{3}+q^{2}+q$, again a contradiction. 
The main result of this section is the following theorem:

Theorem 9.5. Let $G=S p_{2 n}(q)$ with $n \geq 3$ and $(n, q) \neq(3,2),(4,2)$. Assume $V$ is an irreducible $\mathbb{F} G$-module satisfying $\left(\mathcal{W}_{2}^{\varepsilon}\right)$ for some $\varepsilon= \pm$. Then there is a formal sum $W$ of trivial and linear-Weil modules of $G$ if $\varepsilon=+$ and of trivial and unitaryWeil modules of $G$ if $\varepsilon=-$, such that $V=W$ as $P_{j}$-modules for $1 \leq j \leq n-1$ and as $H_{d}$-modules for $1 \leq d \leq n / 2$.

Proof. By Theorem 8.4, there is a formal sum $W$ of trivial and Weil modules of $G$ such that $V=W$ as $P_{1}$-modules and as $H_{d}$-modules for $1 \leq d \leq n / 2$. It remains to prove that $V=W$ as $P_{j}$-modules for $1 \leq j \leq n-1$. We proceed by induction on $j \geq 1$, with the induction base $j=1$ already established. For the induction step assume $n>j \geq 2$ and that $V=W$ as $P_{i}$-modules for $1 \leq i \leq j-1$. We will use the decomposition (42) for $V$ and $W$. Since $Q_{j}<P_{1}, V=W$ as $Q_{j}$-modules. Thus the irreducible $Q_{j}$-characters occurring in $V$ and in $W$ are exactly the same.

1) First we show that $V_{0}=W_{0}$ as $P_{j}$-modules. Let $\lambda \in \operatorname{IBr}_{\ell}\left(Q_{j} / Z_{j}\right)$ be any character occurring in $W_{0}$. By Proposition 9.4, $K_{\lambda}:=\operatorname{Stab}_{P_{j}}(\lambda)$ is contained in a conjugate of some $P_{i}$ with $i<j$. It follows by the induction hypothesis that $V=W$ as $K_{\lambda}$-modules. But $Q_{j} \triangleleft K_{\lambda}$ and $K_{\lambda}$ preserves $\lambda$, so $V_{\lambda}=W_{\lambda}$ as $K_{\lambda}$-modules by Lemma 2.3 Let $\mathcal{O}$ be the $P_{j}$-orbit of $\lambda$ and let $V_{\mathcal{O}}:=\sum_{\mu \in \mathcal{O}} V_{\mu}, W_{\mathcal{O}}:=\sum_{\mu \in \mathcal{O}} W_{\mu}$. Then $V_{\mathcal{O}}=\operatorname{Ind}_{K_{\lambda}}^{P_{j}}\left(V_{\lambda}\right)=\operatorname{Ind}_{K_{\lambda}}^{P_{j}}\left(W_{\lambda}\right)=W_{\mathcal{O}}$ as $P_{j}$-modules. This is true for any orbit $\mathcal{O}$ occurring in $W_{0}$ (and $V_{0}$ ), whence $V_{0}=W_{0}$ as $P_{j}$-modules.

2) Next we show that $V_{k}=W_{k}$ as $P_{j}$-modules for $k=1$, 2. If $k=j$, then $k=j=$ 2 and we are done by Proposition 9.3. So we may assume that $k<j$. Let $\lambda=\lambda_{B} \in$ $\operatorname{IBr}_{\ell}\left(Z_{j}\right)$ be any character occurring in $W_{k}$, and let $g=\operatorname{diag}\left(A, C,{ }^{t} A^{-1}\right) \in I_{\lambda}$. By the definition of $W_{k}, q_{B}$ is a quadratic form of rank $k$ on $\mathbb{F}_{q}^{j}$; in particular $\operatorname{rad}\left(q_{B}\right)$ has dimension $j-k$. Since $A \in O\left(q_{B}\right), A$ preserves $\operatorname{rad}\left(q_{B}\right)$. It follows that any $g \in I_{\lambda}$ preserves a $(j-k)$-dimensional subspace $M^{\prime}$ of $\left\langle e_{1}, \ldots, e_{j}\right\rangle_{\mathbb{F}_{q}}$. It is clear that $M^{\prime}$ is totally isotropic and that $Q_{j}$ acts trivially on $M^{\prime}$. It follows that $K_{\lambda}:=\operatorname{Stab}_{P_{j}}(\lambda)$ is contained in a conjugate of $P_{j-k}$ and so $V=W$ as $K_{\lambda^{-}}$ modules. But $Z_{j} \triangleleft K_{\lambda}$ and $K_{\lambda}$ preserves $\lambda$, so $V_{\lambda}=W_{\lambda}$ as $K_{\lambda}$-modules by Lemma 2.3. Arguing as in 1) we come to the conclusion that $V_{k}=P_{k}$ as $P_{j}$-modules.

Note that 1) and 2) are also valid if $j=n$.

3) From 1) and 2) we obtain that $\bigoplus_{k=0}^{2} V_{k}=\bigoplus_{k=0}^{2} W_{k}$ as $L_{j}$-modules. But $L_{j}<H_{j}$ and $V=W$ as $H_{j}$-modules by the choice of $W$ (notice that we use $j<n$ here). It now follows from (42) that $C_{V}\left(Q_{j}\right)=C_{W}\left(Q_{j}\right)$ as $L_{j}$-modules. Since $Q_{j}$ acts trivially on both $C_{V}\left(Q_{j}\right)$ and $C_{W}\left(Q_{j}\right)$, they are equal as $P_{j}$-modules as well. Consequently, $V=W$ as $P_{j}$-modules.

\section{Restrictions to tori $T_{+}$And $T_{-}$}

In this section we use Deligne-Lusztig theory to handle the tori $T_{\varepsilon}$ of order $q^{n}-\varepsilon$ of $G$. Recall that $T_{\varepsilon}$ is chosen to be a torus of $S L_{2}\left(q^{n}\right)$ which is naturally embedded in $G$.

10.1. Some generalities. We may consider $G$ as the fixed point subgroup $\mathcal{G}^{F}$ for some Frobenius map $F$ on a simple algebraic group $\mathcal{G}$ of type $C_{n}$ in characteristic 2. Let $\mathcal{T}_{\varepsilon}$ be an $F$-stable maximal torus of $\mathcal{G}$ such that $T_{\varepsilon}=\mathcal{T}_{\varepsilon}^{F}$. We begin with the following statements, among which the first one is obvious in the case $q>2$ : 
Lemma 10.1. Let $n \geq 2, \mathcal{T}$ be an $F$-stable maximal torus of $\mathcal{G}$ and $\varepsilon= \pm$.

(i) Assume that $\mathcal{T}^{F} \leq T_{\varepsilon}$ but $\mathcal{T}$ is not $\mathcal{G}^{F}$-conjugate to $\mathcal{T}_{\varepsilon}$. Then $q=2, \mathcal{T}$ is maximally split, and $\mathcal{T}^{F}=1$.

(ii) Let $n$ be an odd prime and $(n, q) \neq(3,2)$. Assume that $\left|\mathcal{T}^{F}\right|=\left|T_{\varepsilon}\right|$. Then $\mathcal{T}$ is $\mathcal{G}^{F}$-conjugate to $\mathcal{T}_{\varepsilon}$.

Proof. (i) In the case $q>2$ all maximal tori of $G$ are nondegenerate (see the proof of [C, Prop. 3.6.6]), whence $\mathcal{T}$ is the only maximal torus of $\mathcal{G}$ that contains $\mathcal{T}^{F}$. So if $\mathcal{T}^{F} \leq\left(\mathcal{T}^{\prime}\right)^{F}$ for some $F$-stable maximal torus $\mathcal{T}^{\prime}$ of $\mathcal{G}$, then $\mathcal{T}^{\prime}=\mathcal{T}$, and so we are done. We will however consider the general case.

Recall that the $\mathcal{G}^{F}$-classes of $F$-stable maximal tori of $\mathcal{G}$ are classified by $F$ conjugacy classes of the Weyl group $W(\mathcal{G})$ of $\mathcal{G}$; cf. [C, Prop. 3.3.3]. In our particular case, $F$ acts trivially on $W(\mathcal{G})$, and each conjugacy class in $W(\mathcal{G})$ contains an element which is a product of positive cycles of length $a_{1}, \ldots, a_{r}$ and negative cycles of length $b_{1}, \ldots, b_{s}$ with $\sum_{i=1}^{r} a_{i}+\sum_{j=1}^{s} b_{j}=n$. Moreover, for the corresponding torus $\mathcal{T}$ we have $\mathcal{T}^{F}=\prod_{i=1}^{r} A_{i} \times \prod_{j=1}^{s} B_{j}$ with $A_{i} \simeq \mathbb{Z}_{q^{a_{i}-1}}$ and $B_{j} \simeq \mathbb{Z}_{q^{b_{j}+1}}$; see for instance [FLT, p. 16,17]. Now we assume that $\mathcal{T}^{F} \leq T_{\varepsilon}$. If $b_{1}=n$, then $\left|\mathcal{T}^{F}\right|=q^{n}+1>\left|T_{+}\right|$, whence $\varepsilon=-$ and $\mathcal{T}$ is $\mathcal{G}^{F}$-conjugate to $\mathcal{T}_{\varepsilon}$, a contradiction. Assume $a_{1}=n$. Then $\left|\mathcal{T}^{F}\right|=q^{n}-1 \geq 3$ divides $\left|T_{\varepsilon}\right|=q^{n}-\varepsilon$, whence $\varepsilon=+$ and $\mathcal{T}$ is $\mathcal{G}^{F}$-conjugate to $\mathcal{T}_{\varepsilon}$, again a contradiction. We may therefore assume that $r+s \geq 2$. In this case each $g \in A_{i}$ has eigenvalue 1 (with multiplicity $2\left(n-a_{i}\right)$ ) on the natural module $\tilde{M}$ of $\mathcal{G}$. But the only element in $T_{\varepsilon}$ with eigenvalue 1 on $\tilde{M}$ is 1 . Since $A_{i} \leq T_{\varepsilon}$, it follows that $A_{i}=1$, i.e. $q=2$ and $a_{i}=1$. The same argument shows that there is actually no $B_{j}$ in $\mathcal{T}^{F}$. We conclude that $q=2, \mathcal{T}^{F}$ is maximally split, and $\mathcal{T}^{F}=1$.

(ii) First assume that $n=3$. Then there are $10 \mathcal{G}^{F}$-classes of $F$-stable maximal tori $\mathcal{T}$ in $\mathcal{G}$, with $\left|\mathcal{T}^{F}\right|=(q-1)^{3},(q-1)^{2}(q+1)$ (2 classes), $(q+1)^{2}(q-1)(2$ classes $),(q-1)\left(q^{2}+1\right),(q+1)\left(q^{2}+1\right),(q+1)^{3}, q^{3}-1$, and $q^{3}+1$; cf. [Lu, p. 87]. Since $q \geq 4$, it is easy to check that these 8 orders are pairwise distinct, and so we are done. Next assume that $n \geq 5,\left|\mathcal{T}^{F}\right|=\left|T_{\varepsilon}\right|$, and we keep the notation in the proof of (i) for $\mathcal{T}^{F}$. Then $q^{n}-\varepsilon=\prod_{i=1}^{r}\left(q^{a_{i}}-1\right) \cdot \prod_{j=1}^{s}\left(q^{b_{j}}+1\right)$. If $a_{1}=n$, then $\varepsilon=+$ and $\mathcal{T}$ is $\mathcal{G}^{F}$-conjugate to $\mathcal{T}_{\varepsilon}$. Similarly, if $b_{1}=n$, then $\varepsilon=-$ and $\mathcal{T}$ is $\mathcal{G}^{F}$-conjugate to $\mathcal{T}_{\varepsilon}$. So we may assume $r+s \geq 2$; in particular, $a_{i}, b_{j}<n$ for all $i, j$. Since $n \geq 5$ is a prime, by [Zs] there is a prime divisor $r$ of $q^{n}-1$ which does not divide $\prod_{i=1}^{n-1}\left(q^{i}-1\right)$ if $\varepsilon=+$, and a prime divisor $r$ of $q^{2 n}-1$ which does not divide $\prod_{i=1}^{2 n-1}\left(q^{i}-1\right)$ if $\varepsilon=-$. Clearly, $r$ divides $\left|T_{\varepsilon}\right|$ but not $\left|\mathcal{T}^{F}\right|$, a contradiction.

Recall that to each $F$-stable maximal torus $\mathcal{T}$ of $\mathcal{G}$ and a character $\theta \in \operatorname{Irr}\left(\mathcal{T}^{F}\right)$ one can define the Deligne-Lusztig (virtual) character $R_{\mathcal{T}, \theta}$; cf. [C] DM]. The characters $R_{\mathcal{T}, \theta}$ are parametrized by the $\mathcal{G}^{F}$-conjugacy classes of pairs $(\mathcal{T}, \theta)$. Let $\mathcal{G}^{*}$ be a simple algebraic group with a Frobenius map $F^{*}$ such that $\left(\mathcal{G}^{*}, F^{*}\right)$ is dual to $(\mathcal{G}, F)$. Then the $\mathcal{G}^{F}$-conjugacy classes of $(\mathcal{T}, \theta)$ are in a bijective correspondence $\Pi$ with the $\mathcal{G}^{* F^{*}}$-conjugacy classes of pairs $\left(\mathcal{T}^{*}, s\right)$, where $s \in \mathcal{G}^{* F^{*}}$ is semisimple and $\mathcal{T}^{*}$ is a $F^{*}$-stable maximal torus containing $s$ [DM, Prop. 13.13]. This correspondence $\Pi$ is explicitly described in [Lu, p. 23, 24]. The set $\operatorname{Irr}\left(\mathcal{G}^{F}\right)$ is partitioned into Lusztig series $\mathcal{E}\left(\mathcal{G}^{F},(s)\right.$ ) (cf. [DM, p. 107]) which run over all geometric conjugacy classes $(s)$ of semisimple elements $s \in \mathcal{G}^{* F^{*}}$. On the other hand, if $s \in \mathcal{G}^{* F^{*}}$ is semisimple, then the rational series $\mathcal{E}\left(\mathcal{G}^{F},(s)_{\mathcal{G}^{* F^{*}}}\right)$ consists of 
all irreducible constituents of those $R_{\mathcal{T}, \theta}$ such that $(\mathcal{T}, \theta)$ correspond to $\left(\mathcal{T}^{*}, s^{\prime}\right)$ with $s^{\prime}$ being $\mathcal{G}^{* F^{*}}$-conjugate to $s$. (Informally speaking, we say that such $R_{\mathcal{T}, \theta}$ belong to $\mathcal{E}\left(\mathcal{G}^{F},(s)_{\mathcal{G}^{* F^{*}}}\right)$.) In our particular situation, since $Z(\mathcal{G})$ is connected, the Lusztig series $\mathcal{E}\left(\mathcal{G}^{F},(s)\right)$ and the rational series $\mathcal{E}\left(\mathcal{G}^{F},(s)_{\mathcal{G}^{*} F^{*}}\right)$ are the same (cf. [DM, p. 136]), hence we will use the notation $\mathcal{E}\left(\mathcal{G}^{F},(s)\right)$ to denote the corresponding rational series.

In general, the Deligne-Lusztig characters $R_{\mathcal{T}, \theta}$ span only a subspace of the space of all class functions on $G$, and any class function on $G$ that is orthogonal to all Deligne-Lusztig characters (with respect to the usual scalar product $(\cdot, \cdot)_{G}$ ) is called an orthogonal function (Senkrechtfunktion in $[\mathrm{Lu}]$ ).

Lemma 10.2. (i) Let $f$ be any orthogonal function. Then $f(t)=0$ for any semisimple element $t \in G$.

(ii) Assume $Z(\mathcal{G})$ is connected and $\chi \in \operatorname{Irr}\left(\mathcal{G}^{F}\right) \cap \mathcal{E}\left(\mathcal{G}^{F},(s)\right)$. Then $\chi$ is a linear combination of those $R_{\mathcal{T},{ }_{\theta}}$ belonging to $\mathcal{E}\left(\mathcal{G}^{F},(s)\right)$ and some orthogonal functions.

Proof. (i) Let $t \in G$ be semisimple and let $\psi$ be the class function which takes value 1 on $t^{G}$ and value 0 otherwise. By [C] Prop. 7.5.5], $\psi$ is a uniform function, hence $\psi$ is orthogonal to $f$. Thus

$$
0=(f, \psi)_{G}=\frac{1}{|G|} \sum_{g \in G} f(g) \overline{\psi(g)}=\frac{1}{|G|} \sum_{g \in t^{G}} f(g)=\frac{\left|t^{G}\right| \cdot f(t)}{|G|}=\frac{f(t)}{\left|C_{G}(t)\right|},
$$

i.e. $f(t)=0$.

(ii) Let $I:=\operatorname{Irr}\left(\mathcal{G}^{F}\right) \cap \mathcal{E}\left(\mathcal{G}^{F},(s)\right), I^{\prime}:=\operatorname{Irr}\left(\mathcal{G}^{F}\right) \backslash I, J:=\left\{R_{\mathcal{T}, \theta} \mid R_{\mathcal{T}, \theta} \in\right.$ $\left.\mathcal{E}\left(\mathcal{G}^{F},(s)\right)\right\}$, and $J^{\prime}:=\left\{R_{\mathcal{T}, \theta} \mid R_{\mathcal{T}, \theta} \notin \mathcal{E}\left(\mathcal{G}^{F},(s)\right)\right\}$. Clearly, $I \perp I^{\prime}, J \subseteq\langle I\rangle_{\mathbb{C}}$, and $J^{\prime} \subseteq\left\langle I^{\prime}\right\rangle_{\mathbb{C}}$ (as Lusztig series form a partition of $\operatorname{Irr}\left(\mathcal{G}^{F}\right)$ ). Write $\langle I\rangle_{\mathbb{C}}$ as the orthogonal sum $\langle J\rangle_{\mathbb{C}} \oplus S$. Then any function $f \in S$ is orthogonal to $J$ and also to $J^{\prime}$, so $f$ is an orthogonal function. Now any $\chi \in I$ can be written as $\alpha+\beta$, where $\alpha \in\langle J\rangle_{\mathbb{C}}$ and $\beta \in S$, and so we are done.

Remark 10.3. Assume that $(\mathcal{G}, F)$ and $\left(\mathcal{G}^{*}, F^{*}\right)$ are dual to each other, with corresponding dual rational maximal tori $\mathcal{T}$ and $\mathcal{T}^{*}$. Then $\mathcal{T}^{*} F^{*}$ is isomorphic to $\operatorname{Irr}\left(\mathcal{T}^{F}\right)$ (considered under multiplication) (cf. [DM. Prop. 13.11]) and the above correspondence $\Pi$ specifies an isomorphism between them.

10.2. The case $n$ is an odd prime. Throughout this subsection we assume that $n$ is an odd prime and $(n, q) \neq(3,2)$. Let $G_{\ell}$ denote the set of $G$-conjugacy classes of $\ell^{\prime}$-elements. The torus $T_{+}$has a unique subgroup $T_{+}^{1} \simeq \mathbb{Z}_{q-1}$, and similarly the torus $T_{-}$has a a unique subgroup $T_{-}^{1} \simeq \mathbb{Z}_{q+1}$. Let $\mathcal{X}_{\ell}$ denote the set of $G$-conjugacy classes of $\ell^{\prime}$-elements that intersect either $T_{+} \backslash T_{+}^{1}$ or $T_{-} \backslash T_{-}^{1}$. Since $n$ is assumed to be an odd prime, one can check that the elements in $T_{+} \backslash T_{+}^{1}$ split into $\frac{1}{2 n}\left(q^{n}-q\right)$ $G$-classes, with representatives $h_{28}(i), 1 \leq i \leq\left(q^{n}-q\right) / 2 n$ (where we keep the notation consistent with the one used in [Lu] for $n=3$ ), and the centralizer of any of them in $G$ equals $T_{+}$. Similarly, the elements in $T_{-} \backslash T_{-}^{1}$ split into $\frac{1}{2 n}\left(q^{n}-q\right)$ $G$-classes, with representatives $h_{31}(i), 1 \leq i \leq\left(q^{n}-q\right) / 2 n$, and the centralizer of any of them in $G$ equals $T_{-}$. The elements in $T_{+}^{1} \backslash\{1\}$ split into $\frac{q-2}{2} G$-classes, with representatives $h_{5}(i), 1 \leq i \leq(q-2) / 2$, and the elements in $T_{-}^{1} \backslash\{1\}$ split into $\frac{q}{2}$ $G$-classes, with representatives $h_{6}(i), 1 \leq i \leq q / 2$.

In our case $\mathcal{G}$ is of type $C_{n}$ and $q$ is even, so $\mathcal{G}^{F} \simeq \mathcal{G}^{* F^{*}}$. Let $g_{8}(i)$, resp. $g_{9}(i)$, $g_{31}(i), g_{34}(i)$, be representatives of the $\mathcal{G}^{* F^{*}}$-classes corresponding to the $G$-classes 
of $h_{5}(i), h_{6}(i), h_{28}(i), h_{31}(i)$, respectively (again we keep the notation consistent with the one used in [ $[\mathrm{Lu}]$ for $n=3)$. In particular, $C_{\mathcal{G}^{*} F^{*}}\left(g_{k}(i)\right)$ is isomorphic to $G L_{n}(q)$, resp. $G U_{n}(q), T_{+}$, or $T_{-}$, if $k=8,9,31$, or 34 , resp.

For any semisimple $\ell^{\prime}$-element $s \in \mathcal{G}^{* F^{*}}$, let

$$
\mathcal{E}_{\ell}\left(\mathcal{G}^{F},(s)\right)=\bigcup_{t \in C_{\mathcal{G}^{*} F^{*}}(s), t \text { any } \ell \text {-element }} \mathcal{E}\left(\mathcal{G}^{F}, s t\right) .
$$

A fundamental result of Broué and Michel [BM] asserts that $\mathcal{E}_{\ell}\left(\mathcal{G}^{F},(s)\right)$ is a union of $\ell$-blocks. Abusing notation, we will also let $\mathcal{E}_{\ell}\left(\mathcal{G}^{F},(s)\right)$ denote the set of all irreducible $\ell$-Brauer characters that belong to this union of $\ell$-blocks.

Let $S_{\ell}$ be the set of $\ell^{\prime}$-elements $1 \neq s \in \mathcal{G}^{* F^{*}}$ that are $\mathcal{G}^{* F^{*}}$-conjugate to any of the above elements $g_{8}(i), g_{9}(i), g_{31}(i), g_{34}(i)$, and let

$$
\mathcal{S}_{\ell}=\left(\bigcup_{s \in S_{\ell}} \mathcal{E}_{\ell}\left(\mathcal{G}^{F},(s)\right)\right) \cap \operatorname{IBr}_{\ell}\left(\mathcal{G}^{F}\right)
$$

Lemma 10.4. Let $G=S p_{2 n}(q), n$ an odd prime, $(n, q) \neq(3,2)$, and keep the above notation. The restriction of functions from $\mathcal{S}_{\ell}$ to $\mathcal{X}_{\ell}$ spans the space $F$ of class functions on $\mathcal{X}_{\ell}$.

Proof. Abusing notation, in this proof we denote by $\hat{\chi}$ the restriction of any class function $\chi$ on $G$ or on $G_{\ell}$ to $\mathcal{X}_{\ell}$. Clearly, $F$ is spanned by $\left\{\hat{\psi} \mid \psi \in \operatorname{IBr}_{\ell}(G)\right\}$. By a result of Geck and Hiss GH], $\left\{\hat{\chi} \mid \chi \in \mathcal{E}\left(\mathcal{G}^{F},(s)\right) \cap \operatorname{Irr}(G)\right\}$ form a basic set for the Brauer characters in $\mathcal{E}_{\ell}\left(\mathcal{G}^{F},(s)\right)$. Hence it suffices to show that for any semisimple $\ell^{\prime}$-element $s \in \mathcal{G}^{* F^{*}}$ and any $\chi \in \mathcal{E}\left(\mathcal{G}^{F},(s)\right), \hat{\chi}$ belongs to $F_{0}:=\left\langle\hat{\psi} \mid \psi \in \mathcal{S}_{\ell}\right\rangle_{\mathbb{C}}$.

1) All classes in $\mathcal{X}_{\ell}$ are semisimple, whence all orthogonal functions vanish at them by Lemma 10.2(i). Furthermore, if $\mathcal{T}$ is not $\mathcal{G}^{F}$-conjugate to $\mathcal{T}_{\varepsilon}$ for any $\varepsilon$, then $R_{\mathcal{T}, \theta}(g)=0$ for any $g \in \mathcal{X}_{\ell}$. For, assume the contrary: $R_{\mathcal{T}, \theta}(g) \neq 0$ for some $g \in \mathcal{X}_{\ell}$. Since $C_{\mathcal{G}}(g)$ is connected, C, Theorem 7.2.8] yields

$$
R_{\mathcal{T}, \theta}(g)=\frac{1}{\left|C_{G}(g)\right|} \sum_{x \in G, x^{-1} g x \in \mathcal{T}^{F}} \theta\left(x^{-1} g x\right) Q_{x \mathcal{T} x^{-1}}^{C_{\mathcal{G}}(g)}(1) .
$$

Since $R_{\mathcal{T}, \theta}(g) \neq 0$, it follows that there is an $x \in G$ such that $x^{-1} g x \in \mathcal{T}^{F}$. Without loss we may replace $g$ by $x^{-1} g x$ and assume $g \in \mathcal{T}^{F}$. In this case $\mathcal{T}^{F} \leq C_{G}(g)=T_{\varepsilon}$ for some $\varepsilon= \pm$. By Lemma 10.1 (i), $\mathcal{T}^{F}=1$, contrary to the fact that $\mathcal{T}^{F} \ni g \neq 1$.

2 ) Here we consider the case $1 \neq s \notin S_{\ell}$. We claim that for any $R_{\mathcal{T}, \theta}$ in $\mathcal{E}\left(\mathcal{G}^{F},(s)\right), \mathcal{T}$ is not $\mathcal{G}^{F}$-conjugate to any $\mathcal{T}_{\varepsilon}$. Indeed, assume to the contrary that $\mathcal{T}$ is $\mathcal{G}^{F}$-conjugate to $\mathcal{T}_{\varepsilon}$ for some $\varepsilon= \pm$. The above correspondence $\Pi$ sends $(\mathcal{T}, \theta)$ to $\left(\mathcal{T}^{*}, s\right)$ and gives an isomorphism $\operatorname{Irr}\left(\mathcal{T}^{F}\right) \simeq \mathcal{T}^{* F^{*}}$; cf. Remark 10.3. Now $\left|\mathcal{T}^{* F^{*}}\right|=\left|\operatorname{Irr}\left(\mathcal{T}^{F}\right)\right|=\left|\mathcal{T}^{F}\right|=\left|T_{\varepsilon}\right|$. By Lemma 10.1(ii) (applied to $\mathcal{G}^{*}$ ), there is a unique $\mathcal{G}^{* F^{*}}$-class of such $\mathcal{T}^{*}$, and this is the class corresponding to $\mathcal{T}_{\varepsilon}$. But $s \in \mathcal{T}^{* F^{*}}$, so in this case $s$ is $\mathcal{G}^{* F^{*}}$-conjugate either to 1 or to an element of $S_{\ell}$, contrary to the assumption we made on $s$.

Now let $\chi \in \mathcal{E}\left(\mathcal{G}^{F},(s)\right)$. By Lemma 10.2(ii), $\chi$ is a linear combination of some orthogonal functions and some $R_{\mathcal{T}, \theta}$ belonging to $\mathcal{E}\left(\mathcal{G}^{F},(s)\right)$. According to 1$)$, the orthogonal functions all vanish on $\mathcal{X}_{\ell}$, and the $R_{\mathcal{T}, \theta}$ 's also vanish on $\mathcal{X}_{\ell}$ since $\mathcal{T}$ is not $\mathcal{G}^{F}$-conjugate to any $\mathcal{T}_{\varepsilon}$. Hence $\hat{\chi}=0$.

3) If $s \in S_{\ell}$, then clearly $\hat{\chi} \in F_{0}$. Finally, we consider the case $s=1$. By Lemma 10.2(ii), $\chi$ is a linear combination of some orthogonal functions and some 
$R_{\mathcal{T}, 1}$. Again, orthogonal functions vanish on $\mathcal{X}_{\ell}$, and $R_{\mathcal{T}, 1}$ vanishes on $\mathcal{X}_{\ell}$ if $\mathcal{T}$ is not $\mathcal{G}^{F}$-conjugate to any $\mathcal{T}_{\varepsilon}$. So it suffices to show that $\hat{R}_{\mathcal{T}, 1}$ belongs to $F_{0}$ when $\mathcal{T}$ is $\mathcal{G}^{F}$-conjugate to $\mathcal{T}_{\varepsilon}$.

Assume for instance that $\mathcal{T}$ is $\mathcal{G}^{F}$-conjugate to $\mathcal{T}_{+}$. We may assume that $\mathcal{T}^{F}=$ $T_{+}$. Let $t$ be a generator of $T_{+} \simeq \mathbb{Z}_{q^{n}-1}, \tilde{t}$ be a primitive $\left(q^{n}-1\right)^{\text {th }}$-root of unity in $\mathbb{C}$, and write $q^{n}-1=\ell^{a} r$ with $(r, \ell)=1$. For $0 \leq i \leq r-1$, let $\theta_{i}$ be the $T_{+}$-character sending $t$ to $\tilde{t}^{{ }^{a} i}$. Let $g \in T_{-} \cap \mathcal{X}_{\ell}$. Since $\left(\left|T_{-}\right|,\left|T_{+}\right|\right)=1$, no $\mathcal{G}^{F}$ conjugate of $g$ can belong to $T_{+}$. Then (43) implies that $R_{\mathcal{T}, 1}$ and $\sum_{i=0}^{r-1} R_{\mathcal{T}, \theta_{i}}$ vanish at $g$.

First we assume $r=1$, i.e. $q^{n}-1=\ell^{a}$. Then every element $h \in \mathcal{X}_{\ell}$ is conjugate to an element $g \in T_{-}$, hence $R_{\mathcal{T}, 1}(h)=0$ due to the above observation. Thus $\hat{R}_{\mathcal{T}, 1}=0$.

Next we assume that $r>1$. We have shown above that $\sum_{i=0}^{r-1} R_{\mathcal{T}, \theta_{i}}$ vanishes at any element $g \in T_{-} \cap \mathcal{X}_{\ell}$. Let $h \in T_{+} \cap \mathcal{X}_{\ell}$. Then $h=t^{\ell^{a} k}$ with $1 \leq k \leq r-1$, and so

$$
\sum_{i=0}^{r-1} \theta_{i}(h)=\sum_{i=0}^{r-1} \tilde{t}^{\ell^{2 a}} k i=\frac{\tilde{t}^{\ell^{2 a} k r}-1}{\tilde{t}^{\ell^{2 a}} k-1}=0 .
$$

Applying (43) to $h$ we get

$$
\sum_{i=0}^{r-1} R_{\mathcal{T}, \theta_{i}}(h)=\frac{1}{\left|C_{G}(h)\right|} \sum_{x \in G, x^{-1} h x \in T_{+}}\left(\sum_{i=0}^{r-1} \theta_{i}\left(x^{-1} h x\right)\right) Q_{x \mathcal{T} x^{-1}}^{C_{\mathcal{G}}(h)}(1)=0 .
$$

Thus $\sum_{i=0}^{r-1} R_{\mathcal{T}, \theta_{i}}$ vanishes at any element $h \in T_{+} \cap \mathcal{X}_{\ell}$. Consequently,

$$
\hat{R}_{\mathcal{T}, 1}=-\sum_{i=1}^{r-1} \hat{R}_{\mathcal{T}, \theta_{i}} .
$$

Observe that for any $i, 1 \leq i \leq r-1, \theta_{i}$ is a nontrivial character of $\mathcal{T}^{F}$ of $\ell^{\prime}$ order (indeed, $\left.\theta_{i}^{r}=1_{\mathcal{T}^{F}}\right)$. So the correspondence $\Pi$ sends $\left(\mathcal{T}, \theta_{i}\right)$ to $\left(\mathcal{T}^{*}, s^{\prime}\right)$, where $s^{\prime} \in \mathcal{T}^{* F^{*}}$ is a nontrivial $\ell^{\prime}$-element. As in 2), the equality $q^{n}-1=\left|\mathcal{T}^{F}\right|=\left|\mathcal{T}^{* F^{*}}\right|$ implies that $\mathcal{T}^{*}$ is $\mathcal{G}^{* F^{*}}$-conjugate to the torus corresponding to $\mathcal{T}_{+}$. Since $s^{\prime}$ is a nontrivial $\ell^{\prime}$-element in $\mathcal{T}^{* F^{*}}, s^{\prime}$ is $\mathcal{G}^{* F^{*}}$-conjugate to $g_{8}(l)$ or $g_{31}(l)$ (for some $l$ ). In other words, $s^{\prime} \in S_{\ell}$. Now $R_{\mathcal{T}, \theta_{i}}$ is certainly a linear combination of some $\chi \in \mathcal{E}\left(\mathcal{G}^{F},\left(s^{\prime}\right)\right) \cap \operatorname{Irr}(G)$, whence $\hat{R}_{\mathcal{T}, \theta_{i}}$ is a linear combination of some $\hat{\psi}$ with $\psi \in \mathcal{E}_{\ell}\left(\mathcal{G}^{F},\left(s^{\prime}\right)\right) \cap \operatorname{IBr}_{\ell}(G)$. It follows that $\hat{R}_{\mathcal{T}, \theta_{i}} \in F_{0}$ for any $i, 1 \leq i \leq r-1$. But in this case (44) implies that $\hat{R}_{\mathcal{T}, 1} \in F_{0}$ as well.

The case when $\mathcal{T}$ is $\mathcal{G}^{F}$-conjugate to $\mathcal{T}_{-}$can be dealt with in the same way.

A crucial ingredient of our argument is

Proposition 10.5. Let $G=S p_{2 n}(q), n$ an odd prime, $(n, q) \neq(3,2)$, and keep the above notation. Let $\varphi$ be a class functions on $G_{\ell}$. Assume that $\varphi \in\left\langle\operatorname{IBr}_{\ell}(G) \backslash \mathcal{S}_{\ell}\right\rangle_{\mathbb{C}}$ and that $\varphi=0$ on $G_{\ell} \backslash \mathcal{X}_{\ell}$. Then $\varphi=0$.

Proof. Let $g_{1}, \ldots, g_{m}$ be representatives of all $G$-classes in $\mathcal{X}_{\ell}$. By Lemma 10.4 there are $\psi_{1}, \ldots, \psi_{m} \in\left\langle\mathcal{S}_{\ell}\right\rangle_{\mathbb{C}}$ such that $\psi_{i}\left(g_{j}\right)=\delta_{i j}$. The aforementioned result of Broué and Michel $[\mathrm{BM}]$ implies that $\operatorname{IBr}_{\ell}(G) \backslash \mathcal{S}_{\ell}$ and $\mathcal{S}_{\ell}$ are disjoint unions of $\ell$-blocks. Hence the orthogonality relations for Brauer characters yield for any $i$ 
that

$$
\begin{aligned}
0 & =\left(\varphi, \psi_{i}\right)_{G}^{\prime}:=\frac{1}{|G|} \sum_{x \in G_{\ell}} \varphi(x) \overline{\psi_{i}(x)} \\
& =\frac{1}{|G|} \sum_{x \in \mathcal{X}_{\ell}} \varphi(x) \overline{\psi_{i}(x)} \\
& =\frac{1}{|G|} \sum_{x \in g_{i}^{G}} \varphi(x)=\frac{\left|g_{i}^{G}\right|}{|G|} \varphi\left(g_{i}\right),
\end{aligned}
$$

i.e. $\varphi\left(g_{i}\right)=0$ for any $i$. Thus $\varphi=0$ on $\mathcal{X}_{\ell}$, whence $\varphi=0$.

Theorem 10.6. Let $G=S p_{2 n}(q), n$ an odd prime, $(n, q) \neq(3,2)$. Assume that $V$ and $W$ satisfy the assumptions (and therefore the conclusions) of Theorem 9.5. Then $V=W$ and $V$ is either the trivial module or a Weil module.

Proof. 1) Again we view $G=\mathcal{G}^{F}$ for some Frobenius map $F$. Then we can find a rational parabolic subgroup $\mathcal{P}$ and its rational Levi subgroup $\mathcal{L}$ such that $P_{1}=\mathcal{P}^{F}$ and $L_{1}=\mathcal{L}^{F}$. In this case, the Lusztig functor $R_{\mathcal{L}}^{\mathcal{G}}$ is just the Harish-Chandra induction $R_{L_{1}}^{G}$, and the Lusztig restriction ${ }^{*} R_{\mathcal{L}}^{\mathcal{G}}$ is the Harish-Chandra restriction ${ }^{*} R_{L_{1}}^{G}$; cf. [DM, p. 48]. Since $Z(\mathcal{G})$ is connected, the Lusztig functor $R_{\mathcal{L}}^{\mathcal{G}}$ respects Lusztig series, i.e. if $s \in \mathcal{L}^{* F^{*}}$ and $\chi \in \mathcal{E}\left(\mathcal{L}^{F},(s)\right)$, then $R_{\mathcal{L}}^{\mathcal{G}}(\chi)$ is a $\mathbb{Z}$-combination of characters from $\mathcal{E}\left(\mathcal{G}^{F},(s)\right)$; cf. [Lu, p. 70].

2) First we consider the case when $V$ satisfies $\left(\mathcal{W}_{2}^{-}\right)$and assume $C_{V}\left(Q_{1}\right)=0$. Then (30) and Corollary 7.5 imply that $a=b=c_{i}=0$ and so $d=0$ (in the notation of the proof of Proposition 8.1), whence $W=0$ (see the definition of $W$ right after (27)), and so $\operatorname{dim}(V)=\operatorname{dim}(W)=0$, a contradiction. Similarly, assume that $V$ satisfies $\left(\mathcal{W}_{2}^{+}\right)$and $C_{V}\left(Q_{1}\right)=0$. Then $C_{W}\left(Q_{1}\right)=C_{V}\left(Q_{1}\right)=0$, whence (37) and Corollary 7.10 imply that $a=b=c_{i}=0$ and so $d=0$ (in the notation of the proof of Proposition 8.3). It follows that $W=0$ (see the definition of $W$ right after (32) $)$, and so $\operatorname{dim}(V)=\operatorname{dim}(W)=0$, again a contradiction.

We have shown that ${ }^{*} R_{L_{1}}^{G}(V)=C_{V}\left(Q_{1}\right) \neq 0$. Since $V$ is an $\mathbb{F} G$-module, we can find an $L_{1}$-simple submodule $U$ of ${ }^{*} R_{L_{1}}^{G}(V)$. By Frobenius' reciprocity, $V$ is a quotient of $R_{L_{1}}^{G}(U)$. The discussion in 1) shows that $R_{L_{1}}^{G}$ respects Lusztig series.

3) Assume $V$ satisfies $\left(\mathcal{W}_{2}^{-}\right)$. By (30) and Corollary [7.5 $U$ can be chosen to have Brauer character $\hat{\rho}-e \cdot 1_{L_{1}}$, where $\rho=1_{L_{1}}, \widehat{\alpha}_{n-1} \otimes 1_{T_{1}}, \widehat{\beta}_{n-1} \otimes 1_{T_{1}}$, or $\hat{\zeta}_{n-1}^{i} \otimes 1_{T_{1}}$, and $e \in \mathbb{Z}$. In the first three cases, $\rho$ is a unipotent character of $L_{1}$; define $s \in \mathcal{L}^{* F^{*}}$ to be 1 . In the last case, item 2) in the proof of Corollary 7.5 shows that $\rho \in \mathcal{E}\left(\mathcal{L}^{F},(s)\right)$, where $s$ corresponds to some element of the form $\operatorname{diag}\left(\xi^{j}, \xi^{-j}, I_{2 n-4}\right)$ of $L_{1}^{\prime}$. Clearly, in all cases the $\ell^{\prime}$-part of $s$ does not belong to $S_{\ell}$. Hence, $V$ belongs to $\operatorname{IBr}_{\ell}(G) \backslash \mathcal{S}_{\ell}$. Also, recall that $W$ is defined to be the virtual $\mathbb{F} G$-module, with character $a \widehat{\alpha}_{n}+b \widehat{\beta}_{n}+\sum_{i=1}^{(r-1) / 2} c_{i} \hat{\zeta}_{n}^{i}+d \cdot 1_{G}$. Clearly, $\widehat{\alpha}_{n}$, $\widehat{\beta}_{n}$, and $1_{G}$ are unipotent, and $\hat{\zeta}_{n}^{i}$ is in $\mathcal{E}_{\ell}\left(\mathcal{G}^{F},\left(s^{\prime}\right)\right)$ with $s^{\prime}$ corresponds to some element of the form $\operatorname{diag}\left(\xi^{j^{\prime}}, \xi^{-j^{\prime}}, I_{2 n-2}\right)$ of $G$, whence all irreducible constituents of them belong to $\operatorname{IBr}_{\ell}(G) \backslash \mathcal{S}_{\ell}$.

4) Assume $V$ satisfies $\left(\mathcal{W}_{2}^{+}\right)$. By (37) and Corollary [7.10, $U$ can be chosen to have Brauer character $\hat{\rho}-e \cdot 1_{L_{1}}$, where $\rho=1_{L_{1}^{\prime}} \otimes \delta_{i}, 1_{L_{1}}, \hat{\rho}_{n-1}^{1} \otimes 1_{T_{1}}, \hat{\rho}_{n-1}^{2} \otimes 1_{T_{1}}$, $\hat{\tau}_{n-1}^{i} \otimes 1_{T_{1}}$, and $e \in \mathbb{Z}$. In the first case, $V$ is a quotient of $R_{L_{1}}^{G}\left(1_{L_{1}^{\prime}} \otimes \delta_{i}\right)=\tau_{n}^{i}$, 
so $V$ belongs to $\operatorname{IBr}_{\ell}(G) \backslash \mathcal{S}_{\ell}$. In the next three cases, $\rho$ is a unipotent character of $L_{1}$; define $s \in \mathcal{L}^{* F^{*}}$ to be 1 . In the last case, item 2) in the proof of Corollary 7.10 shows that $\rho \in \mathcal{E}\left(\mathcal{L}^{F},(s)\right)$, where $s$ corresponds to some element of the form $\operatorname{diag}\left(\delta^{j}, \delta^{-j}, I_{2 n-4}\right)$ of $L_{1}^{\prime}$. Clearly, in the last four cases the $\ell^{\prime}$-part of $s$ does not belong to $S_{\ell}$. Hence, $V$ belongs to $\operatorname{IBr}_{\ell}(G) \backslash \mathcal{S}_{\ell}$. Also, recall that $W$ is defined to be the virtual $\mathbb{F} G$-module, with character

$$
a \hat{\rho}_{n}^{1}+b \hat{\rho}_{n}^{2}+\sum_{i=1}^{(r-1) / 2} c_{i} \hat{\tau}_{n}^{i}+\left(d-a-b-2 \sum_{i=1}^{(r-1) / 2} c_{i}\right) \cdot 1_{G} .
$$

Clearly, $\hat{\rho}_{n}^{1}, \hat{\rho}_{n}^{2}$, and $1_{G}$ are unipotent, and $\hat{\tau}_{n}^{i}$ is in $\mathcal{E}_{\ell}\left(\mathcal{G}^{F},\left(s^{\prime}\right)\right)$ with $s^{\prime}$ corresponding to some element of the form $\operatorname{diag}\left(\delta^{j^{\prime}}, \delta^{-j^{\prime}}, I_{2 n-2}\right)$ of $G$, whence all irreducible constituents of them belong to $\operatorname{IBr}_{\ell}(G) \backslash \mathcal{S}_{\ell}$.

5) We have shown in 3) and 4) that $\varphi, \psi \in\left\langle\operatorname{IBr}_{\ell}(G) \backslash \mathcal{S}_{\ell}\right\rangle_{\mathbb{C}}$ if $\varphi$ is the Brauer character of $V$ and $\psi$ is the Brauer character of $W$. By the conclusion of Theorem 9.5, $\varphi=\psi$ on any $P_{j}$ with $1 \leq j \leq n-1$ and on any $H_{d}$ with $1 \leq d \leq n / 2$. Observe that any element $g \in T_{+}^{1} \cup T_{-}^{1}$ lies in a conjugate of $H_{1}$. From Lemma 2.2(iii), it now follows that $\varphi=\psi$ on $G_{\ell} \backslash \mathcal{X}_{\ell}$. Applying Proposition[10.5 to $\varphi-\psi$, we conclude that $\varphi=\psi$, i.e. $V=W$. Since $V$ is irreducible, the last equality implies that $V$ is either the trivial module or a Weil module.

10.3. Proof of the main theorems. We can now complete the proof of the main results of the paper.

Proof of Theorem 1.2. The cases $n=2$ and $(n, q)=(4,2)$ follow from Proposition 4.1 and Lemma 4.3. Furthermore, the case $n$ is an odd prime follows from Theorems 9.5 and 10.6. We may now assume that $n$ is a composite. Choose $r=2$ if $n$ is even and $r$ to be an odd prime divisor of $n$ if $n$ is odd; in particular, $n=r m$ for some $m>1$.

By Theorem 9.5 there is a formal sum $W$ of trivial and Weil modules of $G$ such that $V=W$ on subgroups $P_{j}$ and $H_{d}$ for $1 \leq j \leq n-1$ and $1 \leq d \leq n / 2$. Embed $S:=S p_{2 r}\left(q^{m}\right)$ naturally in $G$, and define $\kappa=\varepsilon^{m}$. By Lemma 3.7 any composition factor of $\left.W\right|_{S}$ is either a trivial module, or a linear-Weil module (of $S$ ) when $\kappa=+$ and a unitary-Weil module when $\kappa=-$. By Corollary 4.2, $\left.W\right|_{S}$ has property $\left(\mathcal{W}_{2}^{\kappa}\right)$. Consider the $Z_{r}$-subgroup $Z_{r}(S)$ of $S$. Then all $Z_{r}(S)$-characters occurring in $\left.W\right|_{Z_{r}(S)}$ belong to $\mathcal{O}_{1} \cup \mathcal{O}_{2}^{\kappa}$. Since $Z_{r}(S)<P_{1}$ and $V=W$ as $P_{1}$-modules, the same holds for $\left.V\right|_{Z_{r}(S)}$, whence the $S$-module $V$ also satisfies $\left(\mathcal{W}_{2}^{\kappa}\right)$. Observe that $q^{m}>2$. Hence Proposition 4.1, resp. Theorems 9.5 and Theorem 10.6, applies to $S$ when $r=2$, resp. when $r>2$, and yields that any composition factor of $\left.V\right|_{S}$ is either a trivial module, or a linear-Weil module (of $S$ ) when $\kappa=+$ and a unitary-Weil module when $\kappa=-$. Now we consider the $P_{1}$-subgroup $P_{1}(S)$ of $S$. Then $P_{1}(S)$ is contained in a conjugate of $P_{m}$, and $1 \leq m=n / r<n$, whence $V=W$ as $P_{1}(S)$-modules. Thus the $S$-Brauer character $\varphi$ of $V-W$ satisfies the assumptions of Lemma 7.11(i) when $\kappa=+$, and of Lemma 7.11(ii) when $\kappa=-$. By Lemma $7.11 \varphi=0$, i.e. $V=W$ as $S$-modules.

Finally, by Lemma 2.2, any element $g \in G$ is conjugate to an element of some $P_{j}$ with $1 \leq j \leq n-1$, some $H_{d}$ with $1 \leq d \leq n / 2$, or $S$. We conclude that the Brauer characters of $V$ and of $W$ take the same value at any $\ell^{\prime}$-element $g \in G$, i.e. 
$V=W$. Since $V$ is irreducible, the last equality implies that $V$ is either a trivial module or a Weil module.

Proof of Theorem 1.1. The cases $(n, q)=(2,2),(3,2),(4,2)$ can be checked directly using [JLPW], so we assume that $q \geq 4$ if $n \leq 4$.

First we consider the case $n \geq 5$. If $(n, q)=(5,2)$, then $\mathfrak{d}(n, q)=3808$. If $(n, q) \neq(5,2)$, then $\mathfrak{d}(n, q)=b^{-}(n-1, q) \cdot q^{n-1}\left(q^{n-1}-1\right)(q-1) / 2$ (in the notation of Theorem 5.12). By Theorem 5.12 and Remark 5.13] $V$ satisfies $\left(\mathcal{W}_{1}\right)$, whence $V$ satisfies $\left(\mathcal{W}_{2}^{\varepsilon}\right)$ for some $\varepsilon= \pm$ by Theorem 5.11 But in this case $V$ is either the trivial module or a Weil module by Theorem 1.2

Next we assume that $n \leq 4$. If $V$ satisfies $\left(\mathcal{W}_{2}^{\varepsilon}\right)$, then we are done by Theorem 1.2. Assume neither $\left(\mathcal{W}_{2}^{+}\right)$nor $\left(\mathcal{W}_{2}^{-}\right)$holds for $V$. By Lemma 3.4 (i), both $\left(\mathcal{W}_{2}^{+}\right)$ and $\left(\mathcal{W}_{2}^{-}\right)$fail for $\left.V\right|_{Z_{n}}$. In other words, $\left.V\right|_{Z_{n}}$ affords both $\mathcal{O}_{2}^{+}$and $\mathcal{O}_{2}^{-}$. It follows that $\operatorname{dim}(V) \geq\left|\mathcal{O}_{2}^{+}\right|+\left|\mathcal{O}_{2}^{-}\right|=\mathfrak{d}(n, q)$, a contradiction.

Remark 10.7. C. Bonnafé and R. Rouquier (private communication) have recently announced a result establishing a Morita equivalence between nonisolated blocks in $\mathcal{E}_{\ell}\left(\mathcal{G}^{F},(s)\right)$ and $\mathcal{E}_{\ell}\left(\mathcal{L}^{F},(s)\right)$ when $s \in \mathcal{L}^{* F^{*}}$. Suppose that $V$ has property $\left(\mathcal{W}_{2}^{\varepsilon}\right)$. Then Propositions 8.1 and 8.3 and arguments as in the proof of Theorem 10.6 show that either $V$ is in a unipotent block or it is in a nonisolated block. If $V$ is in a nonisolated block, then the announced result of Bonnafé and Rouquier would imply that either $V$ is a Weil module, or $\operatorname{dim}(V)>\mathfrak{d}(n, q)$ (but then one still has to show that the property $\left(\mathcal{W}_{2}^{\varepsilon}\right)$ implies $\left.\operatorname{dim}(V)<\mathfrak{d}(n, q)\right)$. More importantly, unipotent blocks are isolated, and, as it happens most of the time, dealing with unipotent blocks is the most difficult step in solving various problems in the cross characteristic representation theory of finite groups of Lie type.

\section{REFERENCES}

[Atlas] J. H. Conway, R. T. Curtis, S. P. Norton, R. A. Parker, and R. A. Wilson, 'An ATLAS of Finite Groups', Clarendon Press, Oxford, 1985. MR 88g:20025

[BM] M. Broué and J. Michel, Blocs et séries de Lusztig dans un groupe réductif fini, J. reine angew. Math. 395 (1989), 56 - 67. MR 90b:20037

[BrK] J. Brundan and A. S. Kleshchev, Lower bounds for the degrees of irreducible Brauer characters of finite general linear groups, J. Algebra 223 (2000), 615 - 629. MR 2001f:20014

[C] R. Carter, 'Finite Groups of Lie type: Conjugacy Classes and Complex Characters', Wiley, Chichester, 1985. MR 87d:20060

[DM] F. Digne and J. Michel, 'Representations of Finite Groups of Lie Type', London Mathematical Society Student Texts 21, Cambridge University Press, 1991. MR 92g:20063

[DT] N. Dummigan and Pham Huu Tiep, Lower bounds for the minima of certain symplectic and unitary group lattices, Amer. J. Math. 121 (1999), 889 - 918. MR 2001a:11112

[E] H. Enomoto, The characters of the finite symplectic groups $S p(4, q), q=2^{f}$, Osaka J. Math. 9 (1972), 75 - 94. MR 46:1893

[FLT] P. Fleischmann, W. Lempken, and Pham Huu Tiep, The $p$-intersection subgroups in quasi-simple and almost simple finite groups, J. Algebra 207 (1998), 1 - 42. MR 99j:20021

[GH] M. Geck and G. Hiss, Basic sets of Brauer characters of finite groups of Lie type, J. reine angew. Math. 418 (1991), 173 - 188. MR 92e:20006

[Ge] P. Gérardin, Weil representations associated to finite fields, J. Algebra 46 (1977), 54 101. MR 57:470 
[GMST] R. M. Guralnick, K. Magaard, J. Saxl, and Pham Huu Tiep, Cross characteristic representations of odd characteristic symplectic groups and unitary groups, J. Algebra $\mathbf{2 5 7}$ (2002), $291-347$.

[GPPS] R. M. Guralnick, T. Penttila, C. Praeger, and J. Saxl, Linear groups with orders having certain large prime divisors, Proc. London Math. Soc. 78 (1999), 167 - 214. MR 99m:20113

[GT] R. M. Guralnick and Pham Huu Tiep, Low-dimensional representations of special linear groups in cross characteristic, Proc. London Math. Soc. 78 (1999), 116 - 138. MR 2000a:20016

[HM] G. Hiss and G. Malle, Low dimensional representations of special unitary groups, $J$. Algebra 236 (2001), 745 - 767. MR 2001m:20019

[Ho] C. Hoffman, Cross characteristic projective representations for some classical groups, $J$. Algebra 229 (2000), 666 - 677. MR 2001f:20029

[Hw1] R. Howe, On the characters of Weil's representations, Trans. Amer. Math. Soc. 177 (1973), 287 - 298. MR 47:5180

[Hw2] R. Howe, $\theta$-series and invariant theory, Proc. Symp. Pure Math. 33 (1979), part 1, pp. 257 - 285. MR 81f:22034

[Is] I. M. Isaacs, Characters of solvable and symplectic groups, Amer. J. Math. 95 (1973), 594 - 635. MR 48:11270

[JLPW] C. Jansen, K. Lux, R. A. Parker, and R. A. Wilson, 'An ATLAS of Brauer Characters', Oxford University Press, Oxford, 1995. MR 96k:20016

[KLi] P. B. Kleidman and M. W. Liebeck, 'The Subgroup Structure of the Finite Classical Groups', London Math. Soc. Lecture Note Ser. no. 129, Cambridge University Press, 1990. MR 91g:20001

[Ku] S. Kudla, Seesaw dual reductive pairs, in: 'Automorphic Forms of Several Variables', Taniguchi Symposium, Katata, 1983, Birkhäuser, Boston, 1983, pp. 244 - 268. MR 86b:22032

[LaS] V. Landazuri and G. M. Seitz, On the minimal degrees of projective representations of the finite Chevalley groups, J. Algebra 32 (1974), 418 - 443. MR 50:13299

[LST] J. M. Lataille, P. Sin and Pham Huu Tiep, The modulo 2 structure of rank 3 permutation modules for odd characteristic orthogonal groups, J. Algebra 268 (2003), 463 - 483.

[Li] M. W. Liebeck, Permutation modules for rank 3 symplectic and orthogonal groups, $J$. Algebra 92 (1985), 9 - 15. MR 86d:20057

[L] G. Lusztig, 'Characters of Reductive Groups over a Finite Field', Annals of Math. Studies 107, Princeton Univ. Press, Princeton, 1984. MR 86j:20038

$[\mathrm{Lu}] \quad$ F. Lübeck, Charaktertafeln für die Gruppen $C S p_{6}(q)$ mit ungeradem $q$ und $S p_{6}(q)$ mit geradem q, Preprint 93-61, IWR Heidelberg, 1993.

[MT1] K. Magaard and Pham Huu Tiep, Irreducible tensor products of representations of finite quasi-simple groups of Lie type, in: 'Modular Representation Theory of Finite Groups', M. J. Collins, B. J. Parshall, L. L. Scott, eds., Walter de Gruyter, Berlin et al, 2001, pp. 239 - 262. MR 2002m:20024

[MT2] K. Magaard and Pham Huu Tiep, The classes $\mathcal{C}_{6}$ and $\mathcal{C}_{7}$ of maximal subgroups of finite classical groups, (in preparation).

[MMT] K. Magaard, G. Malle, and Pham Huu Tiep, Irreducibility of tensor squares, symmetric squares, and alternating squares, Pacific J. Math. 202 (2002), 379 - 427. MR 2002m:20025

[S] G. M. Seitz, Some representations of classical groups, J. London Math. Soc. 10 (1975), 115 - 120. MR 51:5789

[SS] J. Saxl and G. M. Seitz, Subgroups of algebraic groups contaning regular unipotent elements, J. London Math. Soc. 55 (1997), 370 - 386. MR 98m:20057

[SZ] G. M. Seitz and A. E. Zalesskii, On the minimal degrees of projective representations of the finite Chevalley groups, II, J. Algebra 158 (1993), 233 - 243. MR 94h:20017

[ST] P. Sin and Pham Huu Tiep, Rank 3 permutation modules of finite classical groups, (submitted).

[T] Pham Huu Tiep, Dual pairs and low-dimensional representations of finite classical groups, (in preparation).

[TZ1] Pham Huu Tiep and A. E. Zalesskii, Minimal characters of the finite classical groups, Comm. Algebra 24 (1996), 2093 - 2167. MR 97f:20018 
[TZ2] Pham Huu Tiep and A. E. Zalesskii, Some characterizations of the Weil representations of the symplectic and unitary groups, J. Algebra 192 (1997), 130 - 165. MR 99d:20074

[Wh1] D. White, Decomposition numbers of $S p_{4}\left(2^{a}\right)$ in odd characteristics, J. Algebra 177 (1995), 264 - 276. MR 96k:20023

[Wh2] D. White, Decomposition numbers of unipotent blocks of $S_{6}\left(2^{a}\right)$ in odd characteristics, J. Algebra 227 (2000), 172 - 194. MR 2001d:20012

[Zs] K. Zsigmondy, Zur Theorie der Potenzreste, Monath. Math. Phys. 3 (1892), 265 - 284.

Department of Mathematics, University of Southern California, Los Angeles, CalIFORNIA 90089-2532

E-mail address: guralnic@math.usc.edu

Department of Mathematics, University of Florida, Gainesville, Florida 32611

E-mail address: tiep@math.ufl.edu 